\title{
Exemplarische Anwendung
}

Das in Kapitel 4 beschriebene methodische Vorgehen ist in diesem Kapitel auf drei exemplarische Metalle - Kupfer, Tantal und Kobalt - angewandt. Die dabei generierten Ergebnisse liefern Antworten zu den Forschungsfragen (1), (2) und (3). Die drei Beispielmetalle sind in den Abschnitten 5.2-5.4 abgehandelt. Jeder dieser Abschnitte erläutert zu Beginn die Bedeutung des jeweiligen Metalls und gibt eine kurze Beschreibung über die Ermittlung dessen sekundärer Quellen. Danach ist eine kurze Beschreibung der wichtigsten Recyclingverfahren und der darauf aufbauenden Recyclingmodelle gegeben. Die Auswertungen der Recyclingmodelle sind nach dem Status Quo sowie nach Parametervariationen vorgenommen und diskutiert. Ebenso die sind die Massenbilanzen und die Ergebnisse der energetischen Optimierung der KEA beschrieben. Auf eine detaillierte Erläuterung der Ermittlung der Datengrundlage der Modelle und Massenbilanzen ist in diesem Kapitel verzichtet. Diese kann dem Appendix II des elektronischen Zusatzmaterials entnommen werden.

\subsection{Auswahl der Metalle}

Die Metalle Kupfer, Tantal und Kobalt sind aufgrund mehrerer Faktoren als beispielhafte Metalle für die nachfolgenden Auswertungen ausgewählt. Ein wesentlicher Aspekt ist ihre aktuelle und vor allem zukünftige Nachfrage. Die Kupferbedarfe werden insbesondere durch die Elektrifizierung, den Ausbau der

Elektronisches Zusatzmaterial Die elektronische Version dieses Kapitels enthält Zusatzmaterial, das berechtigten Benutzern zur Verfügung steht https://doi.org/10.1007/978-3-658-32924-2_5 
Erneuerbaren Energien und die damit verbundene Netzinfrastruktur überproportional ansteigen (Elshkaki et al. 2016). Der Bedarf an Tantal ist im Wesentlichen auf die Nachfrage nach Kondensatoren in elektrischen Geräten und Superlegierungen zurückzuführen. Alleine für diese beiden Anwendungsbereiche ist ein Bedarfsanstieg bis 2035 um den Faktor 4 prognostiziert (Marscheider-Weidemann et al. 2016). Für Kobalt ist sogar ein Wachstum um den Faktor 24 errechnet. Wesentlicher Treiber ist hier der Einsatz in Lithium-Ionen Batterien für die automobile Anwendung (Marscheider-Weidemann et al. 2016). Die bereits hohe wirtschaftliche Bedeutung aller drei Metalle wird demnach in Zukunft noch entscheidend zunehmen.

Ein weiterer Aspekt ist, dass die ausgewählten Metalle exemplarisch für bestimmte Metallgruppen herangezogen werden können. Kupfer ist ein Industriemetall mit sehr guten Recyclingeigenschaften und entsprechend hohen EoL-RQ (Graedel et al. 2011; Langner 2011). Tantal, ein Technologiemetall, das bedingt durch seine Anwendungsbereiche meist sehr gering in sekundären Quellen konzentriert ist, weist nur sehr niedrige EoL-RQ auf (Graedel et al. 2011). Kobalt ist insbesondere aufgrund des enormen zukünftigen Bedarfs für hochtechnologische Anwendungen - insbesondere Batterietechniken - als kritisches Metall für den europäischen Wirtschaftsraum eingestuft (EC 2018). Auch bei diesem Metall sind die Konzentrationen in den verfügbaren Quellen meist relativ gering. Dennoch sind die EoL-RQ von Kobalt aktuell relativ hoch (Graedel et al. 2011).

Alle drei Metalle kommen in ihren sekundären Quellen in niedrigeren aber auch in höheren Konzentrationen als in ihren primären Quellen vor. Das ist in Abbildung 5.1 veranschaulicht. Die Ordinate gibt die Konzentrationsverhältnisse der Metalle an (sekundär/primär). Beim Faktor 1 sind die Konzentrationen der primären und sekundären Quellen identisch. Ein Faktor kleiner 1 bedeutet eine geringere Konzentration in der sekundären Quelle und entsprechend vice versa. Dieser Aspekt ist insbesondere vor dem in Abschnitt 3.2 beschriebenen Zusammenhang zwischen der Metallkonzentration und dem Energiebedarf der Metallgewinnung interessant.

Die in Abbildung 5.1 aufgeführten sekundären Quellen sind die mengenmäßig relevantesten für die Metalle Kupfer, Tantal und Kobalt. Eine genaue Beschreibung der sekundären Quellen, deren Kennzahlen sowie die Begründung ihrer Auswahl ist in den jeweiligen Unterkapiteln aufgeführt. 


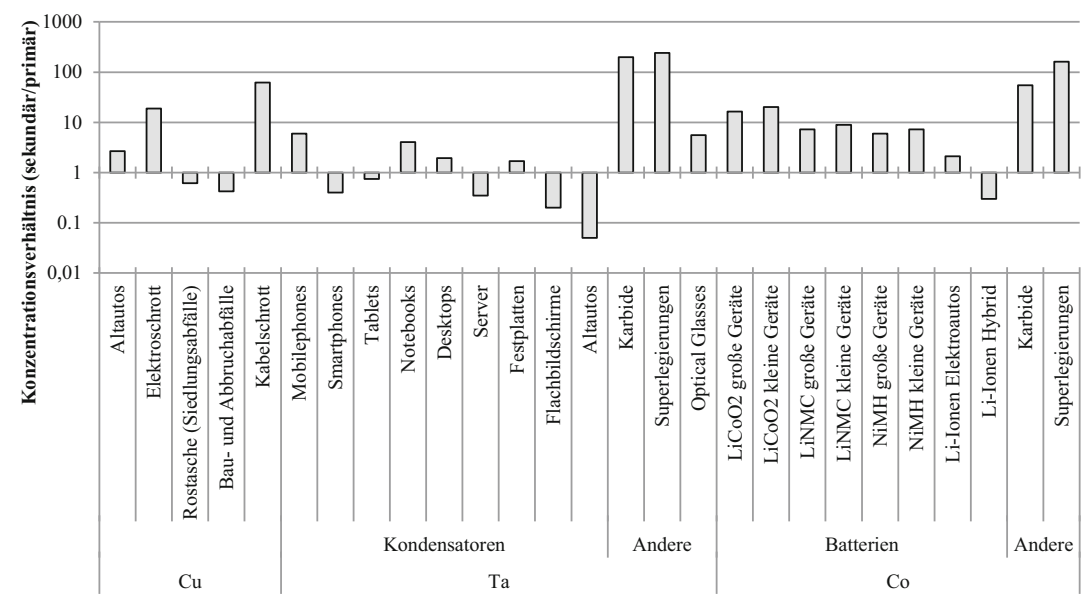

Abbildung 5.1 Konzentrationsverhältnis der sekundären und primären Quellen der Metalle Kupfer, Tantal und Kobalt ( $>1$ sekundäre Quelle höher konzentriert und vice versa; die Datengrundlage kann Appendix I und II des elektronischen Zusatzmaterials entnommen werden)

\section{$5.2 \quad$ Kupfer}

\subsubsection{Die Bedeutung von Kupfer}

Kupfer ist insbesondere aufgrund seiner inhärenten Eigenschaften, die eine gute Verarbeitung, Korrosionsresistenz und eine hohe thermische Leitfähigkeit ermöglichen, eines der ersten Metalle, das die Menschheit aktiv zum Gebrauch herangezogen hat. Seine Nutzung geht bis auf 10.000 Jahre v. Ch. zurück. Mit der fortschreitenden Erforschung und vermehrten Nutzung der Elektrizität rückte ab dem Ende des 18. Jahrhunderts eine weitere zentrale Eigenschaft von Kupfer in den Mittelpunkt - die elektrische Leitfähigkeit. Mit 59,1 × $10^{6}$ Siemens pro Meter (S/m) liegt Kupfer nur knapp hinter Silber mit $61,5 \times 10^{6} \mathrm{~S} / \mathrm{m}$ und ist damit das Element der Erde mit der zweitbesten elektrischen Leitfähigkeit. Mit deutlichem Abstand folgt Aluminium $\left(36,6 \times 10^{6} \mathrm{~S} / \mathrm{m}\right)$ auf Platz 3. Somit gibt es für Kupfer kein wirkliches Substitut, das einen moderaten Marktpreis hat, um den enormen Bedarf an leitfähigem Material decken zu können und gleichzeitig nicht in zu großen Leitungsverlusten resultiert (Langner 2011). 
Heute besitzt Kupfer als Industriemetall eine solch hohe Bedeutung für das ökonomische, soziale und ökologische System wie nie zuvor. Alleine im Jahr 2018 wurden 21 Mio. t Kupfer aus primären Quellen gefördert - mehr als jemals in einem einzigen Jahr zuvor (USGS 2019). Die Abhängigkeit der Wirtschaft von Kupfer ist offensichtlich - beinahe jede ökonomische Aktivität benötigt elektrische Energie, deren Bereitstellung und vor allem Distribution, wie oben erläutert, ohne Kupfer kaum möglich wäre. Zudem bedarf eine enorme Bandbreite an Produkten der spezifischen Eigenschaften von Kupfer. Allen voran natürlich die elektrische Leitfähigkeit, aber auch z. B. die Korrosionsbeständigkeit oder die hohe thermische Leitfähigkeit. Die Abhängigkeit der Gesellschaft bzw. des sozialen Systems von Kupfer ist ebenfalls leicht zu erkennen. Der Einsatz von Kupfer ist eine zentrale Notwendigkeit, um ausreichende Mengen an elektrischer Energie und elektronischen Produkten bereitzustellen, die eine moderne Kommunikation, Unterhaltung, Mobilität und entsprechenden Komfort ermöglichen.

Neben der allgemeinen wirtschaftlichen und gesellschaftlichen Bedeutung kommt Kupfer zudem eine besondere Rolle in der nachhaltigen Entwicklung zu. Wesentliche Maßnahmen einer nachhaltigen Entwicklung sind der Umschwung auf Erneuerbare Energien und die Elektrifizierung des Verkehrs. In beiden Fällen ist Kupfer unabdingbar (Elshkaki et al. 2016; Schipper et al. 2018).

Abschnitt 3.1.1 zeigt, dass noch enorme Mengen an abbauwürdigem Kupfer in primären Quellen vermutet werden. Dennoch ist Kupfer mit einer mittleren Konzentration in der Erdkruste von ca. 0,005 \% für ein Industriemetall relativ selten (Dorner et al. 2013a). Aufgrund dieser Tatsache, sowie der hohen wirtschaftlichen Bedeutung, ist Kupfer in zahlreichen Studien mit einer mittleren Kritikalität eingestuft (Erdmann und Behrendt 2011; Kroop et al. 2014; Graedel et al. 2015).

\subsubsection{Sekundäre Kupferquellen}

In Deutschland wurden im Jahr 2014 ca. 1.465 .000 t Kupfer verwendet (Wirtschaftsvereinigung Metalle 2016). Diese Kupfermassen lassen sich auf sechs Hauptanwendungsgebiete herunterbrechen - siehe hierzu Abbildung 5.2. In Abhängigkeit der unterschiedlichen Lebensdauern der jeweiligen Anwendungen ist diese Menge Kupfer im anthropogenen Stofflager gebunden und fällt schließlich zu unterschiedlichen Zeitpunkten in sekundären Quellen an.

Rund $81 \%$ der im Jahr 2014 verwendeten Kupfermenge entfiel auf die prozentual größten Anwendungsbereiche Kabel und Elektro, Bauwesen und Automobil. Diese Anwendungsbereiche sind zudem durch eine teils starke räumliche 


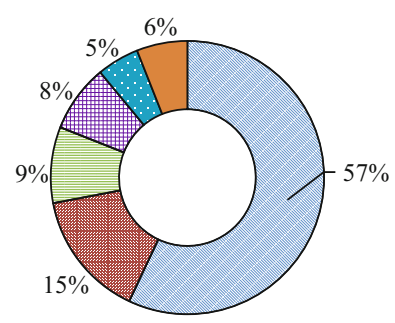

$\square$ Kabel und Elektro

圆Bauwesen

日Automobil

囲 Maschinenbau

$\square$ Handel

$\square$ Sonstiges

Abbildung 5.2 Hauptanwendungsgebiete Kupfer (2014) (GDB 2014)

Verteilung der Produkte in der Nutzungsphase und relativ geringe Kupferkonzentrationen gekennzeichnet, was sie für die vorgesehene Untersuchung besonders interessant und relevant macht. Die Maschinenbaubranche ist aus mehreren Gründen nicht in die Untersuchung mitaufgenommen. Grundsätzlich funktioniert das Recycling von EoL-Produkten, die in einem unternehmerischen Bereich anfallen, meist sehr koordiniert und zu hohen Quoten (Graedel et al. 2011). Allerdings ist bei betriebsinternen Prozessen meist keine ausreichende und zugängliche Datenlage vorhanden. Zudem liegt bei Produkten der Maschinenbaubranche für gewöhnlich kein Kupferkonzentrationsproblem (Ruhrberg 2006) und kein Verteilungsproblem vor, was die Relevanz für die vorgesehenen Untersuchungen schmälert. Durch den relativ geringen Massenanteil am Gesamtkupferverbrauch und die durchweg langen Lebenszeiten der Maschinenbauprodukte ist zudem der Einfluss auf die Massenbilanz des für ein Recycling verfügbaren Kupfers in sekundären Quellen marginal. Das Anwendungsfeld Handel fließt ebenfalls nicht in diese Massenbilanz ein. Hier werden keine Produkte hergestellt werden und somit auch keine sekundären Quellen generiert.

Die nachfolgend betrachteten sekundären Quellen sind Altautos, Elektroaltgeräte bzw. Elektroschrott, Kabelschrott, Bau- und Abbruchabfälle sowie Rostasche aus der Verbrennung von Siedlungsabfällen. Letztere ist untersucht, da verschiedenste kupferhaltige Produkte unweigerlich auch Wege in den Gewerbe- oder Hausmüll finden. Zahlreiche Publikationen, die die Massenbilanzen bzw. Stoffströme von Kupfer untersucht haben, verweisen auf die gleichen bzw. sehr ähnliche sekundäre Quellen (Bertram et al. 2002; Ruhrberg 2006; Tanimoto et al. 2010; Vexler et al. 2004; Glöser et al. 2013). Die sekundären Kupferquellen und deren Kupferkonzentrationen $\omega_{C u}\left(x_{i}\right)$ sind in Tabelle 5.1 zusammengefasst. 
Tabelle 5.1 Sekundäre Kupferquellen und deren Kupferkonzentrationen (Datengrundlage in Kapitel B. 1 des Appendix II des elektronischen Zusatzmaterials)

\begin{tabular}{l|l}
\hline sekundäre Quelle & $\omega_{C u}\left(x_{i}\right)[\mathrm{g} / \mathrm{g}]$ \\
\hline Kabelschrott & 0,36 \\
\hline Elektroaltgeräte & 0,1059 \\
\hline Altautos & 0,0155 \\
\hline Rostasche (Siedlungsabfälle) & 0,00355 \\
\hline Bau- und Abbruchabfälle & 0,00245 \\
\hline
\end{tabular}

\subsubsection{Recyclingmodell}

\subsubsection{Recyclingverfahren und Prozessdaten}

Im Gegensatz zu vielen anderen Metallen hat Kupfer einen entscheidenden Vorteil - aufgrund dessen chemischer Eigenschaften kann es durch eine elektrolytische Raffination ohne Qualitätsverluste in höchster Reinheit unendlich oft aufbereitet werden. Dabei werden die in den metallurgisch aufbereiteten Kupferfraktionen enthaltenen Begleitmetalle über die metallurgische Prozesskette nicht nur vom Kupfer abgetrennt, sondern häufig auch als Kuppelprodukte ausgebracht und in entsprechenden Nebenprozessen aufbereitet. Kupfer fungiert dabei für eine Vielzahl an Metallen - insb. Edelmetalle - als Trägermetall (Kawohl 2011; Samuelsson und Björkman 2014; Langner 2011). Die Rückgewinnung der weiteren Metalle als Kuppelprodukte trägt dabei zur Wirtschaftlichkeit des Gesamtprozesses bei (Steinacker et al. 2016). Der Wandel von relativ reinen Kupferschrotten hin zu komplexen sekundären Quellen mit teils bedeutenden Edelmetallgehalten, wie z. B. Elektroaltgeräten (Rombach und Friedrich 2019; Kawohl 2011), beschränkt die Kupferhütten nicht mehr rein auf die Kupferrückgewinnung. Sie sind nun vielmehr auf ein Multi-Metall-Recycling spezialisiert (Schlesinger et al. 2011; Langner 2011).

Kupferschrotte bzw. aufbereitete sekundäre Kupferquellen finden in zwei metallurgischen Verfahrensrouten Einsatz - in der reinen sekundären und in der primären Kupfermetallurgie. In der primären Metallurgie, die, wie der Name vermuten lässt, hauptsächlich Kupfer aus Erzkonzentraten gewinnt, können jedoch nur sehr reine Kupferschrotte eingesetzt werden. Kupferfraktionen mit Konzentrationen unter $90 \%$ müssen über die eigens dafür ausgelegte sekundäre Kupfermetallurgie aufbereitet werden. Beide Verfahrensrouten funktionieren pyrometallurgisch mit einer finalen elektrolytischen Raffination. ${ }^{1}$ Im Falle von

\footnotetext{
${ }^{1}$ Es existieren auch hydrometallurgische Verfahren, über die jedoch großindustriell keine sekundären Quellen aufbereitet werden.
} 
hochreinen Schrotten, kann auf eine pyro- und elektrometallurgische Raffination verzichtet und ein direktes Umschmelzen der Schrotte vorgenommen werden (Schlesinger et al. 2011). Detaillierte Beschreibungen der metallurgischen Prozesse sowie eine Auflistung aller relevanten Prozessdaten können Appendix II des elektronischen Zusatzmaterials entnommen werden.

Das Kupferrecycling aus sekundären Quellen läuft in den meisten Fällen analog zu dem typischen Recyclingverfahrensschema ab, das in Abbildung 2.3 (Abschnitt 2.1) illustriert ist. Altautos, Elektroaltgeräte, Kabelschrotte und Rostaschen werden nach der Sammlung über mechanische und physikalische Trennund Separationsverfahren in unterschiedliche Metallfraktionen getrennt, die wiederum in die entsprechenden metallurgischen Verfahren eingebracht werden. Bauund Abbruchabfälle weichen leicht vom typischen Recyclingverfahren ab. Im Falle dieser sekundären Quelle findet die Sammlung im Grunde zeitgleich mit der mechanischen Aufbereitung statt. Man spricht hier von einem selektiven Rückbau, also einer Separation der sekundären Quellen in die entsprechenden Stofffraktionen am Ort des Anfallens.

Für die meisten Transporte, die für das Kupferrecycling notwendig sind, sind LKWs angenommen. Ausnahmen sind die Sammlungen der Elektroaltgeräte und Altautos. Diese werden typischerweise von den Endkonsumenten per PKW vorgenommen. Die Sammel- und Transportdistanzen sind basierend auf realen Standorten der Sammelstellen und Aufbereitungsbetrieben ermittelt. Ausführliche Beschreibungen der Prozesse, Transporte und der Prozessdatenermittlung sind Kapitel B.1 in Appendix II des elektronischen Zusatzmaterials zu entnehmen. Die generierten sekundärquellenspezifischen Prozessdaten der Modellbildung sind in Tabelle 5.2 enthalten.

\subsubsection{Modellergebnisse}

Abbildung 5.3 zeigt das Modell des Kupferrecyclingsystems, das mit der Software Umberto LCA $+{ }^{\circledR}$ erstellt ist (nachfolgend als Umberto-Modell bezeichnet), in einer Sankey Darstellung. Die grünen Pfeile geben dabei die KEA an. Je breiter ein Pfeil, desto höher ist der KEA. Grüne Kreise stehen für Inputstellen (Energie, Materialien), rote Kreise für Outputstellen (Emissionen, Abfälle, Produkte) eines Prozesses. Gelbe Kreise sind Verbindungsstellen zwischen zwei Prozessen. Vierecke mit blauen Rändern und weißer Füllung stellen die Foregroundprozesse dar, also die eigentlichen Recycling- bzw. Transportprozesse, deren Prozessdaten in Tabelle 5.2 enthalten sind. Vierecke mit blauen Rändern und gelber Füllung sind Backgroundprozesse, die wiederum die Energie- und Materialinputs der Foregroundprozesse bereitstellen und alle damit verbundenen Aufwände (KEA, $\mathrm{CO}_{2}$ eq usw.) abdecken. 


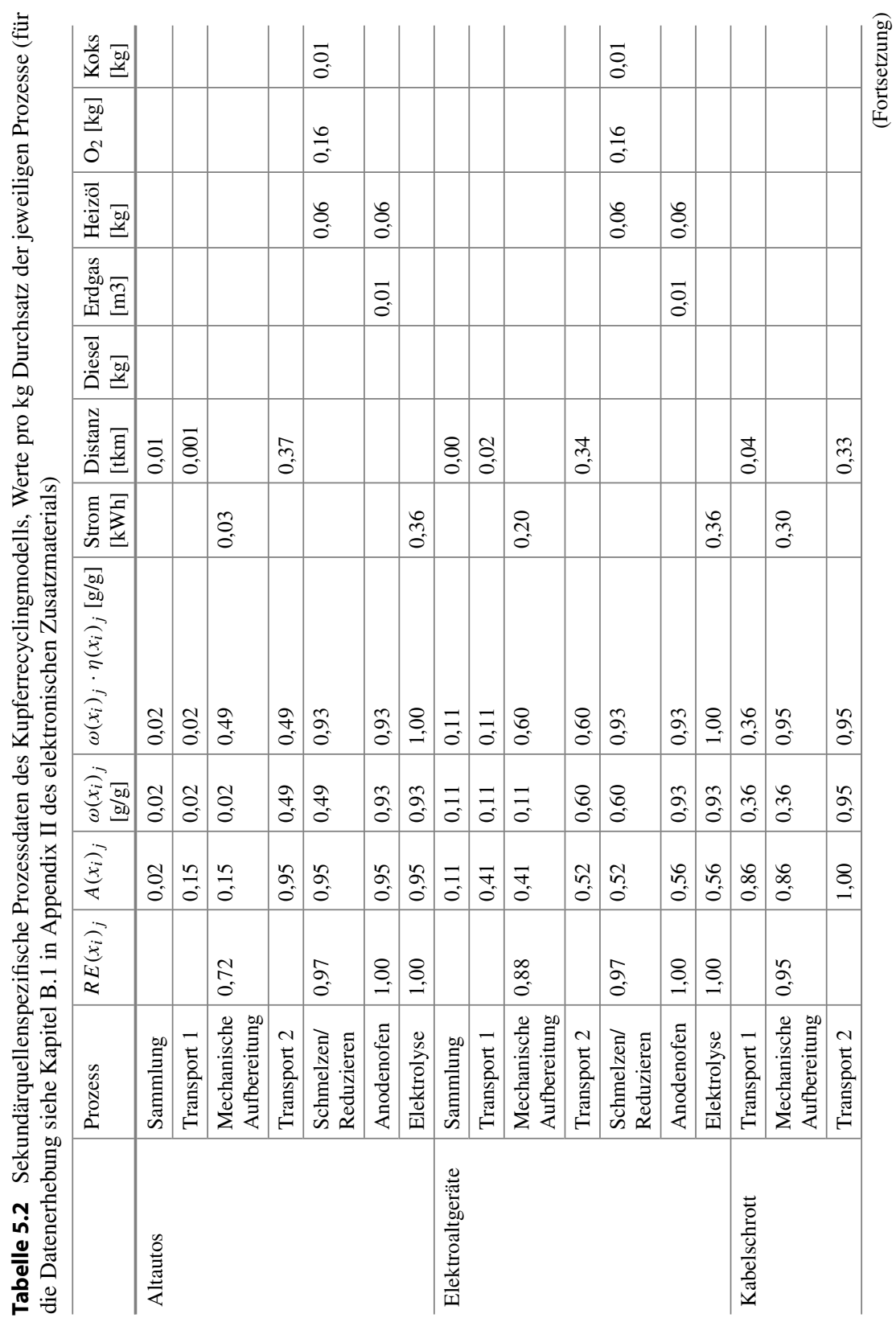




\begin{tabular}{|c|c|c|c|c|c|c|c|c|c|c|c|c|c|c|c|c|}
\hline 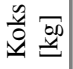 & & & & & & & & & & & & & & $\ddot{\sigma}$ & & \\
\hline $\begin{array}{l}\overrightarrow{b 0} \\
\ddot{y} \\
\tilde{O}\end{array}$ & & & & & & & & & & & & & & $\frac{0}{0}$ & & \\
\hline 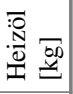 & $\begin{array}{l}8 \\
8 \\
0\end{array}$ & & & & & & $\stackrel{0}{\circ}$ & & $\begin{array}{l}0 \\
0 \\
0\end{array}$ & & & & & $\begin{array}{l}0 \\
0\end{array}$ & $\begin{array}{l}8 \\
0 \\
0\end{array}$ & \\
\hline 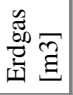 & $\begin{array}{l}0 \\
0 \\
0\end{array}$ & & & & & & $\ddot{0}$ & & $\begin{array}{l}0 \\
0 \\
0\end{array}$ & & & & & & $\begin{array}{l}\sigma \\
0 \\
0\end{array}$ & \\
\hline 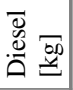 & & & $\tilde{\sigma}_{0}$ & & $\begin{array}{l}\overline{0} \\
0\end{array}$ & & & & & & & & & & & \\
\hline 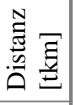 & & & & 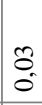 & & $\begin{array}{l}\infty \\
m \\
0 \\
0\end{array}$ & & & & & $\begin{array}{l}0 \\
0 \\
0\end{array}$ & & $\stackrel{2}{\tilde{0}}$ & & & \\
\hline 总 & & $\begin{array}{l}0 \\
\text { m. } \\
0\end{array}$ & 8 & & $\begin{array}{l}8 \\
0\end{array}$ & & & $\begin{array}{l}0 \\
\text { ले } \\
0\end{array}$ & & $\begin{array}{l}0 \\
2 \\
0 \\
0\end{array}$ & & $\ddot{0}$ & & & & ֻ. \\
\hline 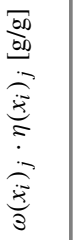 & $\begin{array}{l}n \\
\sigma\end{array}$ & 8 & \&. & §. & $\stackrel{n}{\hat{o}}$ & $\begin{array}{l}\check{2} \\
\hat{\sigma}\end{array}$ & $\hat{\sigma}$ & 8 & $\begin{array}{l}n \\
\sigma \\
0\end{array}$ & $\underset{-1}{8}$ & 8 & $\begin{array}{l}\infty \\
0 \\
0\end{array}$ & $\begin{array}{l}\infty \\
\ddot{0} \\
0^{\circ}\end{array}$ & $\hat{\sigma}$ & $\stackrel{\Omega}{\sigma}$ & 8 \\
\hline $\begin{array}{l}\stackrel{5}{\tilde{3}} \overline{00} \\
300\end{array}$ & $\begin{array}{l}n \\
\alpha \\
0\end{array}$ & $\stackrel{n}{\sigma}$ & $\begin{array}{l}\sigma \\
\delta \\
\delta\end{array}$ & $\begin{array}{l}8 \\
0\end{array}$ & \&. & $\begin{array}{l}n \\
\alpha \\
0\end{array}$ & 8 & $\hat{\sigma}$ & $\tilde{\sigma}$ & $\begin{array}{l}n \\
\sigma \\
0\end{array}$ & $\begin{array}{l}0 \\
\delta \\
0\end{array}$ & $\stackrel{n}{8}$ & $\begin{array}{l}\mathscr{0} \\
\dot{0} \\
0\end{array}$ & $\underset{0}{\infty}$ & $\tilde{\sigma}$ & مू \\
\hline$\stackrel{\vec{\xi}}{\vec{k}}$ & $\underset{-}{8}$ & $\underset{-}{8}$ & ñ & 8 & $\underset{-}{8}$ & $\underset{-}{\stackrel{0}{0}}$ & $\underset{8}{8}$ & $\underset{-}{8}$ & $\underset{-}{8}$ & $\underset{-i}{8}$ & $\begin{array}{l} \pm \\
0 \\
0\end{array}$ & 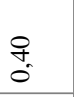 & $\begin{array}{c}n \\
n \\
0 \\
\vdots\end{array}$ & $\begin{array}{l}n \\
n \\
0\end{array}$ & مे & है. \\
\hline 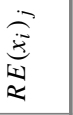 & ळ. & $\underset{-}{8}$ & $\frac{N}{2}$ & & $\bar{\sigma}$ & & $\bar{a}$ & $\underset{-}{8}$ & a. & $\underset{-1}{8}$ & & $\stackrel{\mathscr{\vartheta}}{\ominus}$ & & $\tilde{\sigma}$ & $\underset{-}{8}$ & 8 \\
\hline 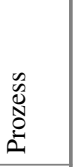 & $\begin{array}{l}\frac{\pi}{0} \\
0 \\
\frac{0}{0} \\
0 \\
0 \\
\vdots \\
\vdots\end{array}$ & 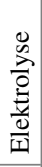 & 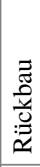 & 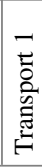 & 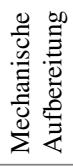 & 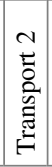 & 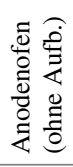 & 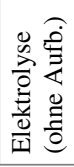 & 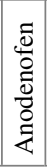 & 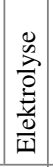 & 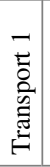 & 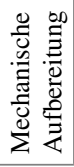 & 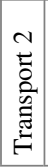 & 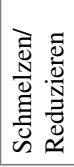 & $\begin{array}{l}\frac{\bar{v}}{0} \\
\frac{0}{0} \\
\frac{0}{0} \\
0 \\
0 \\
\frac{0}{\alpha}\end{array}$ & $\frac{\mathscr{0}}{\stackrel{0}{2}}$ \\
\hline & & & & 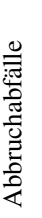 & & & & & & & \multicolumn{6}{|c|}{ 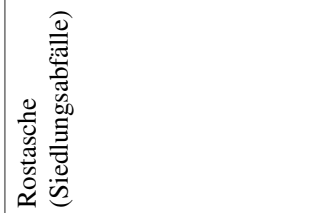 } \\
\hline
\end{tabular}


Die verschiedenfarbigen Bereiche unterteilen die Recyclingkette in die jeweiligen Phasen (Sammlung, Aufbereitung etc.) und ermöglichen so eine differenzierte Auswertung. Die gepunkteten Linien sind ein rein visuelles Hilfsmittel. Sie grenzen die Recyclingverfahren der jeweiligen sekundären Quellen voneinander ab. Die Aufwände des Recyclings der sekundären Quellen sind im abgebildeten Modell auf jeweils $1 \mathrm{~kg}$ recyceltes Kupfer skaliert. Die Breite der grünen Pfeile, die nach der Phase der Metallurgie in einer Outputstelle zusammenlaufen, gibt an, welcher KEA für das Recycling von $1 \mathrm{~kg}$ Kupfer aus den jeweiligen sekundären Quellen benötigt wird. Diese Pfeile sind über die Recyclingverfahren hinweg aus den jeweiligen Backgroundprozessen gespeist. Pfeile, die innerhalb der Phasen die Recyclingkette in eine Outputstelle verlassen, sind auf Allokationen zurückzuführen, also Zurechnungen der Aufwände auf die verschiedenen Kuppelprodukte (siehe auch Abschnitt 4.3.4).

Abbildung 5.3 veranschaulicht, dass das Recycling von Kupfer, selbst aus relativ hoch konzentrierten Quellen wie Elektroaltgeräten oder Kabelschrotten, eine Reihe von Transporten und Aufbereitungsschritten benötigt, die jeweils unterschiedliche KEA erfordern. Beim Recycling der sekundären Quellen Kabelschrott, Bau- und Abbruchabfälle und Rostasche sind jeweils zwei Recyclingpfade modelliert. Bei Kabelschrotten ist der Recyclingpfad von der Qualität der aufbereiteten Kupferfraktionen abhängig. Hochreine Fraktionen können direkt umgeschmolzen werden, weniger reine Fraktionen gehen in die metallurgische Aufbereitung. Bei Bau- und Abbruchabfällen geht ein Teil der rückgebauten kupferhaltigen Fraktionen in eine weitere Aufbereitung. Für das Kupferrecycling aus der sekundären Quelle der Rostasche existieren zwei fundamental unterschiedliche Logistiksysteme.

Die anhand des Kupferrecyclingmodells ermittelten allozierten $K E A_{S}\left(x_{i}\right)$ des Kupferrecyclings sind in Abbildung 5.4 in einem Balkendiagramm veranschaulicht. Die Sammlung weist für alle sekundären Quellen einen nur geringen Anteil an den Gesamtaufwänden auf. Das ist auf die Massenallokation zurückzuführen, die der Sammlung unterstellt ist. Die relativ geringen Kupferkonzentrationen der sekundären Quellen haben somit keinen Einfluss auf die Aufwände der Sammlung. Ein weiterer Grund für die geringen Sammelaufwände ist die ausgeprägte Infrastruktur, die für das Kupferrecycling im Speziellen, aber auch für das Recycling der relevanten sekundären Quellen im Allgemeinen, existiert. So müssen durchschnittlich nur $3 \mathrm{~km}$ bei Elektroaltgeräten und $9 \mathrm{~km}$ bei Altautos zu den Sammelstellen zurückgelegt werden (siehe auch Abschnitt B.1.3.2 in Appendix II des elektronischen Zusatzmaterials). Auch die Aufwände der weiteren Transporte fallen eher gering aus. Gründe dafür sind ebenfalls Allokationseffekte und die gute Recyclinginfrastruktur. 


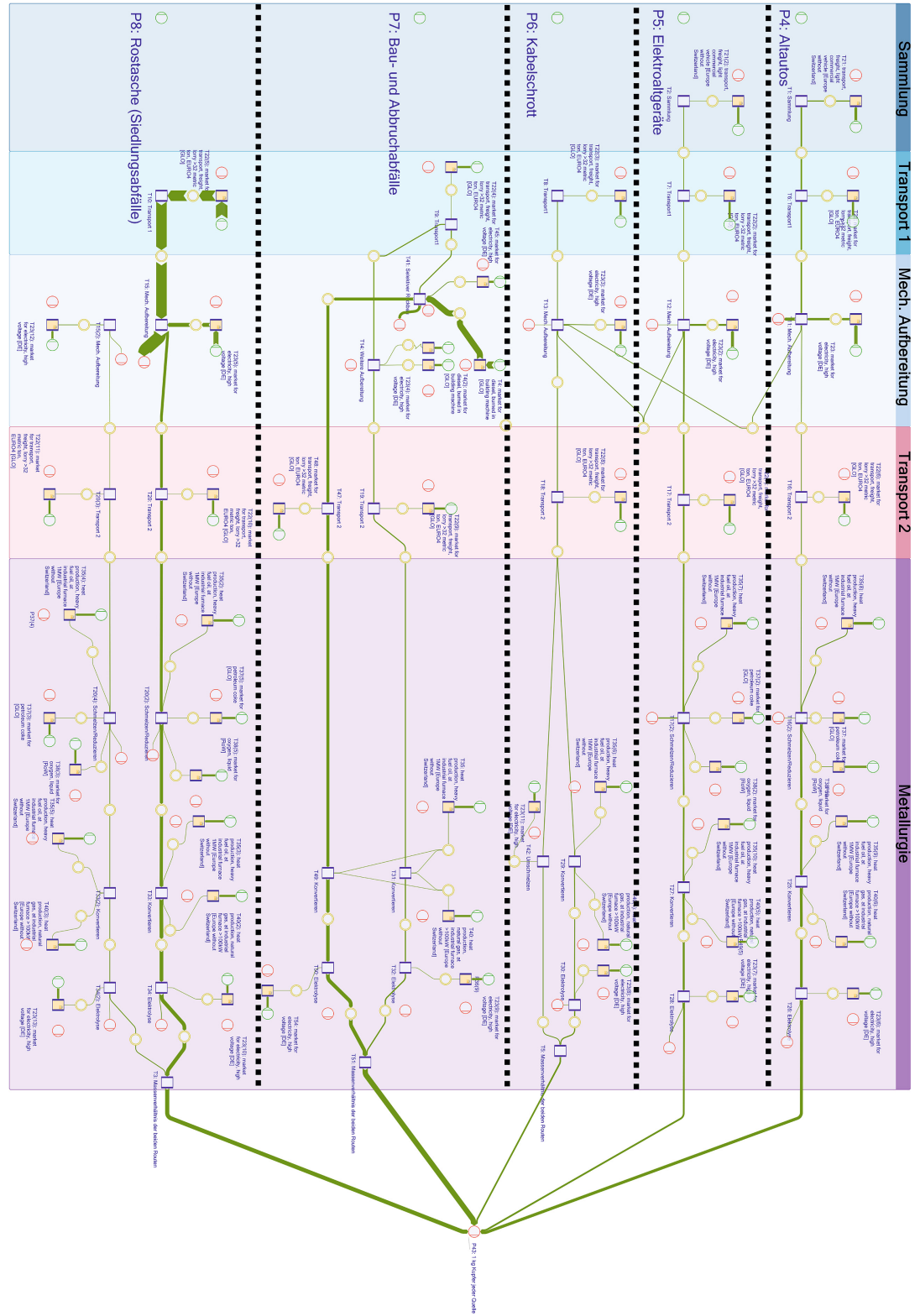

Abbildung 5.3 Umberto-Modell des Kupferrecyclingsystems (Sankey Darstellung der KEA, alloziert) 
Die KEA der mechanischen Aufbereitung fallen bei allen sekundären Quellen relativ hoch aus. Das ist auf die Kupferkonzentrationen zurückzuführen, die am Anfang der Prozesskette noch am geringsten sind und die Aufwände für $1 \mathrm{~kg}$ Kupfer entsprechend hoch ausfallen lassen. Dennoch sind die Aufwände der mechanischen Aufbereitung bereits entscheidend durch Allokationseffekte abgeschwächt, das ist u. a. in Abbildung 5.3 ersichtlich. Eine differenzierte Auflistung aller Modellergebnisse - mit und ohne Allokationen - kann Kapitel C.1 des Appendix III des elektronischen Zusatzmaterials entnommen werden. Welche Aufwände die Metallurgie benötigt, ist durch die Reinheit der Kupferfraktion bestimmt, die in diesen Prozessschritt eingebracht wird. So ist z. B. die Kupferfraktion, die in der Altautoaufbereitung erzeugt wird, verhältnismäßig unrein und weist eher niedrige Kupferkonzentrationen auf. Somit fallen auch die KEA der Metallurgie relativ hoch aus.

Abbildung 5.4 zeigt, dass die $K E A_{S}\left(x_{i}\right)$ des Kupferrecyclings je nach sekundärer Quelle sehr unterschiedlich ausfallen können. Kupferrecycling ist also keineswegs gleich Kupferrecycling. Das Recycling von Kupfer aus Bau- und Abbruchabfällen benötigt die höchsten $K E A_{S}\left(x_{i}\right)$, gefolgt von Rostasche, Altautos und Elektroschrott. Die geringsten $K E A_{S}\left(x_{i}\right)$ und damit auch die geringsten $\mathrm{CO}_{2}$ eq-Emissionen verursacht das Kupferrecycling aus Kabelschrotten. Diese sekundäre Quelle weist auch die höchste Kupferkonzentration auf. Eine Korrelationsanalyse der allozierten $K E A_{S}\left(x_{i}\right)$ des Kupferrecyclings und den Kupferkonzentrationen der sekundären Quellen - siehe Abbildung 5.5 - zeigt einen deutlichen Zusammenhang dieser beiden Werte mit einem Bestimmtheitsmaß von $\mathrm{R}^{2}=0,988$.

Die Auswertung in Abbildung 5.5 zeigt, dass die $K E A_{S}\left(x_{i}\right)$ des Kupferrecyclings bei abnehmenden Konzentrationen über alle sekundären Quellen hinweg einem Potenzwachstum folgen. Im einleitenden Abschnitt 1.1.2 ist aufgezeigt, dass über die vergangenen Jahrzehnte ein Trend der sinkenden Metallkonzentrationen zu beobachten war. In diesem Zusammenhang kommt die Frage auf, wie sich die $K E A_{S}\left(x_{i}\right)$ bei Änderungen der Kupferkonzentrationen in den sekundären Quellen verhalten. Um das zu untersuchen, müssen bestimmte Annahmen getroffen werden. Denn wenn die Konzentration eines Materials - in diesem Fall die Kupferkonzentration - in einer sekundären Quelle sinkt oder steigt, ändern sich automatisch die Konzentrationen aller anderen Materialien in dieser sekundären Quelle. Aktive Konzentrationsänderungen sind ebenfalls denkbar durch z. B. Substitution des Kupfers durch andere Materialien. Selbst wenn Informationen zu den Konzentrationsänderungen gegeben wären, ist nicht bekannt, wie sich die Konzentrationsänderungen auf die Recyclingeffizienzen der einzelnen 


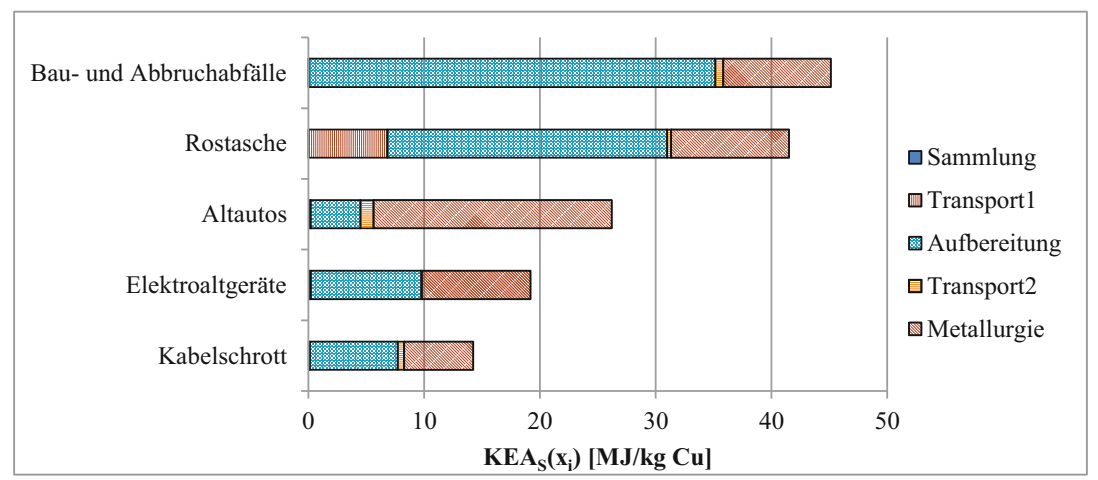

Abbildung 5.4 Allozierte prozessscharfe KEA des Kupferrecyclings aus sekundären Quellen

Metalle und Prozesse auswirken. Somit können auch keine verlässlichen Allokationsfaktoren bestimmt werden. Es sind daher die Annahmen getroffen, dass sowohl die Allokationsfaktoren als auch die Recyclingeffizienzen für Kupfer konstant bleiben. Basierend auf diesen Annahmen kann anhand des parametrisierten Recyclingmodells annähernd ermittelt werden, wie sich die $K E A_{S}\left(x_{i}\right)$ des Kupferrecyclings bei Änderungen der Kupferkonzentration verhalten. Diese Auswertung ist ebenfalls in Abbildung 5.5 veranschaulicht.

Die nach der Kupferkonzentration parametrisierten $K E A_{S}\left(x_{i}\right)$ des Recyclings der fünf sekundären Quellen weisen alle ein Potenzwachstum auf - denn je geringer die Konzentration, desto mehr Masse muss an sekundären Quellen transportiert und aufbereitet werden. Dieser Effekt ist bereits aus der Primärgewinnung von Metallen bekannt (Norgate und Jahanshahi 2010). Die oben getroffenen Annahmen können dabei signifikante Auswirkungen auf die $K E A_{S}\left(x_{i}\right)$ haben. Würden sich die Allokationsfaktoren entsprechend der Änderungen der Konzentrationen anpassen, wären die Auswirkungen auf die $K E A_{S}\left(x_{i}\right)$ geringer. $^{2}$ Die $K E A_{S}\left(x_{i}\right)$ würde also unelastischer auf Konzentrationsänderungen reagieren und die in Abbildung 5.5 abgebildeten Aufwandsfunktionen entsprechend flacher ausfallen. Auffällig ist, dass die Aufwandsfunktionen je nach sekundärer Quelle unterschiedliche Steigungsverläufe aufweisen. Die $K E A_{S}\left(x_{i}\right)$ des Kupferrecyclings aus sekundären Quellen mit hohen Konzentrationen steigen mit

\footnotetext{
${ }^{2}$ Sinken z. B. ausschließlich die Kupferkonzentrationen in einer sekundären Quelle, kann aus dieser Quelle logischerweise nun weniger Kupfer recycelt werden; der Allokationsfaktor für Kupfer sinkt entsprechend.
} 
abnehmender Konzentration stärker an als die des Recyclings aus sekundären Quellen mit geringeren Konzentrationen. D. h. wäre die Konzentration an Kupfer in Kabelschrott so gering wie bspw. in Altautos, dann würde das Kupferrecycling aus Kabelschrott einen höheren $K E A_{S}\left(x_{i}\right)$ benötigen, als es derzeit bei Altautos der Fall ist. Das ist darauf zurückzuführen, dass die sekundären Quellen mit höheren Konzentrationen relativ hohe KEA pro Masseneinheit Durchsatz $\left(K E A_{D}\left(x_{i}\right)\right)$ benötigen. Die geringeren KEA pro Masseneinheit recyceltes Kupfer $\left(K E A_{S}\left(x_{i}\right)\right)$ aus z. B. Kabelschrott oder Elektroaltgeräten resultieren ausschließlich aus deren hohen Kupferkonzentrationen.

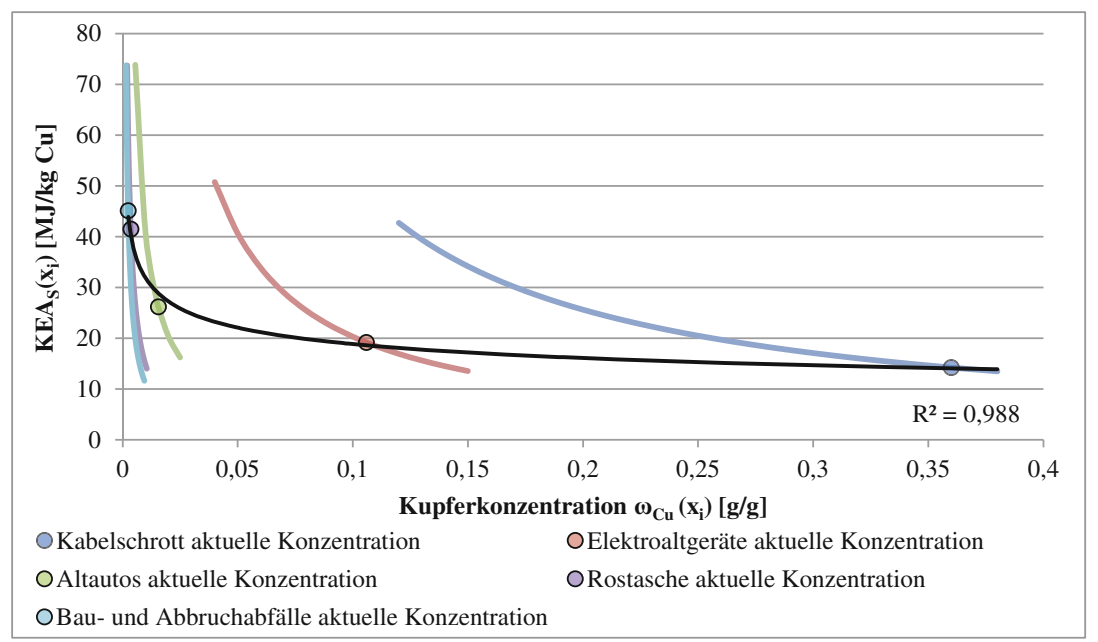

Abbildung 5.5 Auswertung der KEA des Kupferrecyclings nach den Konzentrationen

Ein hoher $K E A_{D}\left(x_{i}\right)$ kann auf unterschiedliche Ursachen zurückgeführt werden. So kann zum einen die Komplexität der sekundären Quelle dafür verantwortlich sein, die ein entsprechend umfangreiches und aufwändiges Recycling benötigt. So weist z. B. eines der führenden deutschen Aufbereitungsunternehmen von Kabelschrott darauf hin, dass Kabel, die zwar auf den ersten Blick nicht sonderlich komplex erscheinen, einen starken Materialverbund aufweisen. Das bezieht sich insbesondere auf die Kunststoffummantelung des Metallkerns. Um diesen Materialverbund aufzutrennen, muss sehr fein zerkleinert werden (siehe auch Abschnitt 2.2), was einen entsprechend hohen Energiebedarf benötigt (Cablo GmbH 2017b). Neben der Komplexität der sekundären Quelle können auch die 
Recyclingeffizienzen der Aufbereitungsprozesse eine Rolle spielen. Hohe Kupferkonzentrationen bedeuten einen hohen ökonomischen Anreiz Kupfer gezielt zu recyceln, und das mit möglichst hohen Ausbeuten. Um möglichst viel Kupfer zu recyceln, müssen die Verluste gering bzw. die Recyclingeffizienzen hochgehalten werden. Das bedeutet einen feineren Aufschluss in der mechanischen Aufbereitung (siehe Abschnitt 2.2) oder die direkte Eingabe der sekundären Quelle zu geringeren Konzentrationen in die Metallurgie, um kein Kupfer in der mechanischen Aufbereitung zu verlieren. In beiden Fällen ist ein Mehraufwand an Energie notwendig, um die höheren Recyclingeffizienzen zu erzielen (Hagelüken 2006). Die Aufwände sind also maßgeblich, aber nicht ausschließlich durch die Konzentrationen bestimmt. Auch die Komplexität der sekundären Quellen und die zu erzielenden Recyclingeffizienzen haben entscheidenden Einfluss.

\subsubsection{Massenbilanzen}

Die Massenbilanzen des Kupferrecyclings beziehen sich auf das Jahr 2014, da hier Aktualität und eine ausreichende Datenlage gegeben sind. Die Massenbilanz basiert auf statistischen Daten, Literaturwerten sowie Primärdaten und Abschätzungen aus der unternehmerischen Praxis. Die Datenerhebung ist in Abschnitt B.1.3.1 in Appendix II des elektronischen Zusatzmaterials beschrieben. Abbildung 5.6 illustriert die Massenbilanz anhand der reinen Kupfermassenströme in einem Sankey-Diagramm. Kupferverluste, die durch unzureichende Sammlung, mechanische Aufbereitung und Metallurgie entstehen, sind differenziert ausgewiesen. Auch die Zusammenhänge der Kupferströme der sekundären Quellen untereinander sind abgebildet. So gehen z. B. geringe Prozentsätze der Elektroaltgeräte nicht in eine Sammlung, sondern in den Hausmüll und enden damit in der Rostasche der Müllverbrennung.

Die inländisch erzeugte Menge an Sekundärkupfer aus sekundären Quellen beträgt 168.242 t für das Jahr 2014. Die sekundären Quellen Kabelschrott, Elektroaltgeräte und Bau- und Abbruchabfälle haben mit jeweils ca. $50.000 \mathrm{t}$ die größten Anteile an der jährlichen Produktion an sekundärem Kupfer. Die Massen an sekundärem Kupfer aus Altautos und Rostasche betragen jeweils ca. 5.000 t. Diese Zahlen stellen jedoch nur eine theoretische Größe dar, da in der Prozesskette der Kupferrückgewinnung an verschiedensten Stellen Im- und Exporte stattfinden (Erdmann et al. 2004), die nicht oder nur unvollständig nachvollzogen und den jeweiligen Abfallgruppen zugeordnet werden können. Nach Angaben des Statistischen Bundesamtes wurden im Jahr 2014658.981 t Kupferschrott importiert und $530.556 \mathrm{t}$ Kupferschrott exportiert (Destatis 2019). Welcher Anteil dabei auf New 
Scrap zurückzuführen ist oder welche tatsächlichen Kupfermengen in den Schrottmengen enthalten sind, kann nicht nachvollzogen werden. Grundsätzlich kann jedoch ein Importüberschuss von 128.425 t Kupferschrott festgehalten werden.

Nach Angaben von Erdmann et al. (2004) sind ca. 1/3 der in Deutschland anfallenden Menge an Kupferschrott auf sekundäre Quellen bzw. Old Scrap und 2/3 auf New Scrap zurückzuführen. Für das Jahr 1999 verweist diese Publikation auf ca. 200.000 t Kupfer als Old Scrap und ca. 400.000 t Kupfer als New Scrap. Aus dieser Publikation geht ebenfalls hervor, dass diese Mengen nicht der tatsächlich im Schrott enthaltenen Menge Kupfer entsprechen, sondern der Gesamtmenge an aufbereitetem Schrott, der einer Verhüttung zugeführt werden kann. Da diese Angaben stark veraltet sind, können sie zwar nicht als aktuelle Vergleichsgröße für die Validität der ermittelten Gesamtbilanz herangezogen werden, zeigen jedoch, dass die ermittelte Masse an sekundärem Kupfer in der richtigen Größenordnung liegt. Aktuellere Werte von Steger et al. (2017) bestätigen ebenfalls die Größenordnung der Ergebnisse.

In Abbildung 5.6 ist deutlich zu erkennen, dass die größten Massenverluste bei der Sammlung bzw. im Falle der Bau- und Abbruchabfälle beim selektiven Rückbau auftreten, also beim ersten Prozessschritt der Recyclingkette. Insbesondere die Sammlungen von Altautos und Elektroaltgeräten weisen hohe Kupferverluste auf. Diese Verluste sind nicht als massenbilanzielle Dissipationen in andere Metallfraktionen zu verstehen, wie es bei der mechanischen und metallurgischen Aufbereitung der Fall ist (siehe auch Abschnitt 2.2), sondern als nicht stattindende Sammlungen oder Sammlungen, die in teils illegalen Exporten in andere Länder resultieren. Insbesondere Elektroaltgeräte finden häufig erst gar nicht den Weg in eine Sammlung, sondern verbleiben im Besitz des Endkonsumenten. Das zeigen Untersuchungen am Beispiel der Mobiltelefone (Welfens et al. 2016; Martinho et al. 2017). Exporte in andere Länder, häufig Entwicklungsoder Schwellenländer, sind bei Elektroaltgeräten und Altautos ebenfalls durchaus üblich (Kohlmeyer et al. 2017; Sander et al. 2017; Sander und Schilling 2010). Zum einen gehen so der deutschen Volkswirtschaft die darin enthaltenen sekundären Rohstoffe verloren, zum anderen werden sie in den Importländern meist mit rudimentären Techniken recycelt, die verhältnismäßig hohe Umweltbelastungen und geringe Metallausbeuten bedeuten (Hagelüken und Meskers 2010).

Die Kupferverluste der mechanischen und metallurgischen Aufbereitung fallen für alle sekundären Quellen prozentual relativ gering aus. Insbesondere die Kupfermetallurgie erzielt für Kupfer, aber auch für die zahlreichen Kuppelprodukte, hohe Ausbeuten bzw. Recyclingeffizienzen von deutlich über $90 \%$ (Aurubis AG 2015, 2017b). So verweisen auch aktuelle Studien, die Empfehlungen zur Prozessoptimierung der Kupfermetallurgie bereitstellen, lediglich auf Möglichkeiten 


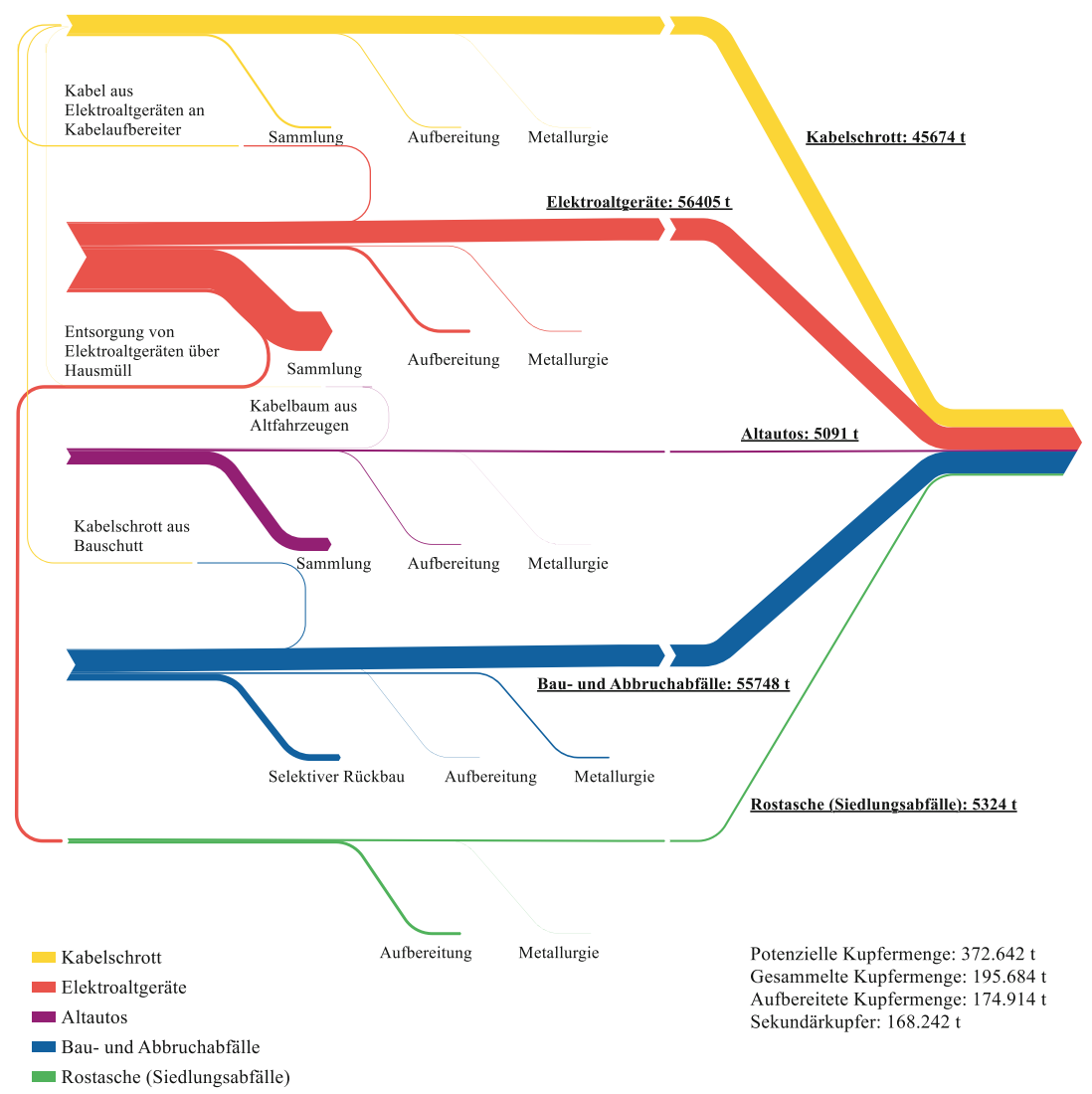

Abbildung 5.6 Massenbilanz des Kupferrecyclings aus sekundären Quellen für das Jahr 2014 in Deutschland

der Emissionsminimierung oder Steigerungen der Energieeffizienz und nicht auf Steigerungen der Metallausbeuten (EC 2017a, 2014a). Auch wenn die prozentualen Verluste der mechanischen und metallurgischen Aufbereitung relativ gering ausfallen, so betragen die absoluten Massen der Kupferverluste ca. 27.500 t. $75 \%$ davon entstehen bereits bei der mechanischen Aufbereitung. Diese Kupferverluste sind nicht nur ein Verlust an potenziellem Sekundärkupfer, sondern eben gleichzeitig potenzielle Verunreinigungen anderer Metallfraktionen (siehe 
Abschnitt 2.2). Je später die Verluste in der Recyclingkette anfallen, desto größer sind zudem die Auswirkungen auf den $K E A_{S}\left(x_{i}\right)$.

\subsubsection{Vergleich der primären und sekundären Gewinnung}

\subsubsection{Aufwände der Primärgewinnung}

Abschnitt 5.2.3 und 5.2.4 enthalten alle notwendigen Daten der sekundären Gewinnung, um die Ermittlung der energetisch optimalen EoL-RQ nach der in Abschnitt 4.2 erläuterten Methode vornehmen zu können. Die Werte der primären Kupfergewinnung, die ebenfalls für diese Analyse benötigt werden, sind in diesem Abschnitt ermittelt. In Abschnitt 4.4.1 ist bereits erläutert, dass aufgrund unterschiedlicher Erzgehalte, Lagerstättentypen und Gewinnungsmethoden die Aufwände der primären Gewinnung stark voneinander abweichen können. Nachfolgend ist daher die Bildung eines entsprechenden Mittelwertes dokumentiert.

Die primäre Kupfergewinnung wird von zwei Verfahren dominiert, die im Wesentlichen durch die Lagerstättentypen d. h. die Beschaffenheit des Kupfererzes bestimmt sind. Sulfidisch gebundene Kupfererze werden üblicherweise über die pyrometallurgische Route gewonnen. Diese Erze machen ca. 80 \% des aktuellen Kupferabbaus aus (Norgate und Jahanshahi 2010). Die übliche Konzentration in den Erzen liegt aktuell bei 0,5-1 \% (Langner 2011). Der zweite derzeit abbaufähige Lagerstättentyp ist mit ca. $20 \%$ das sogenannte oxidische Kupfererz, das durch hydrometallurgische Verfahrensschritte gewonnen werden kann (Norgate und Jahanshahi 2010). In der Literatur werden hierzu Erzgehalte mit einer sehr ähnlichen Spannweite wie die der sulfidischen Erze erwähnt (Giurco 2005; Norgate und Jahanshahi 2010; Northey et al. 2014; Rankin 2012).

Die Unterschiede in den Lagerstättentypen, Erzgehalten und Aufbereitungsverfahren sind der Grund dafür, dass die in der Literatur verfügbaren Energiebedarfe für die Primärgewinnung von Kupfer stark schwanken. Rankin gibt einen KEA von $33 \mathrm{MJ} / \mathrm{kg} \mathrm{Cu}$ für die pyrometallurgische Verarbeitung an, für die hydrometallurgische Verarbeitung weist er einen Wert von $64 \mathrm{MJ} / \mathrm{kg} \mathrm{Cu}$ aus (Rankin 2011, S. 199). Nimmt man ein globales Verhältnis der pyrometallurgischen zur hydrometallurgischen Verarbeitung von $80 \%$ zu $20 \%$ an (Norgate u. Jahanshahi 2010), so ergibt sich ein Gesamtwert von ca. $39 \mathrm{MJ} / \mathrm{kg} \mathrm{Cu}$. In einer anderen Studie wird die pyrometallurgische Route mit rund $130 \mathrm{MJ} / \mathrm{kg} \mathrm{Cu}$ bewertet (Norgate $\mathrm{u}$. Haque 2010). Dieser sehr hohe Wert ist auf das Ignorieren der Kuppelprodukte zurückzuführen, d. h. es wurden hier keine Allokationen vorgenommen. In der Datenbank ecoinvent schwanken die angegebenen Werte je nach geografischer 
Lage zwischen 32 und $69 \mathrm{MJ} / \mathrm{kg} \mathrm{Cu}$ (ecoinvent 2018a, 2018b, 2018c). Die International Copper Association (ICA) gibt einen Wert von $47 \mathrm{MJ} / \mathrm{kg} \mathrm{Cu}$ für den durchschnittlichen nicht-erneuerbaren Primärenergieverbrauch an, der gesamte KEA liegt bei $51 \mathrm{MJ} / \mathrm{kg} \mathrm{Cu}$. Die von der ICA angegebenen Werte beziehen sich dabei auf eine Kombination aus pyro- $u$. hydrometallurgisch gewonnenem Kupfer. Die $\mathrm{CO}_{2}$ eq-Emissionen sind auf $4,1 \mathrm{~kg} / \mathrm{kg} \mathrm{Cu}$ errechnet (ICA 2017). Der wesentliche Vorteil dieses Datensatzes liegt darin, dass ihm eine umfassende Datenbasis zugrunde liegt. 52 Unternehmen vom Bergbau bis zur Metallurgie, verteilt über vier Kontinente, haben Primärdaten zur Erstellung dieses Datensatzes bereitgestellt. Aus allen verfügbaren Quellen bietet er den umfassendsten Wert der globalen Primärkupferproduktion und ist daher für die nachfolgenden Untersuchungen herangezogen.

Ob sich die Kupfermetallurgie in Zukunft verstärkt auf die pyro- oder hydrometallurgische Aufbereitung verschiebt, kann nur schwer prognostiziert werden. Norgate und Jahanshahi (2010) nehmen an, dass ein verstärkter Einsatz der Hydrometallurgie anzunehmen ist, wenn Erze feiner gemahlen werden müssen. Das ist durchaus möglich, sollten die Erzgehalte, die abgebaut werden, auch weiterhin sinken. Es gibt auch durchaus andere Meinungen, die die Pyrometallurgie als weiterhin dominierende Technologie sehen (Langner 2011; Arndt et al. 2017). In diesem Fall würde sich an der aktuellen Situation wenig ändern. Es ist daher der verstärkte Wandel zur Hydrometallurgie als Zukunftsszenario angenommen. Da der Datensatz der ICA nicht auf die hydrometallurgische Route disaggregiert werden kann, ist für dieses Zukunftsszenario der Wert von Rankin (2011, S. 199) mit $64 \mathrm{MJ} / \mathrm{kg} \mathrm{Cu}$ angesetzt. Die $\mathrm{CO}_{2}$ eq-Emissionen liegen bei $6,16 \mathrm{~kg} / \mathrm{kg}$ $\mathrm{Cu}$. Diese Prognose ist jedoch nur eine ungefähre Abschätzung, da sie mögliche Effizienzgewinne der primären Gewinnung über die Zeit nicht berücksichtigt.

\subsubsection{Ermittlung der energetisch optimalen End-of-Life Recyclingquoten}

\subsection{Status Quo und Zukunftsszenario}

Die in Abschnitt 5.2.3.2 ermittelten Aufwände des Kupferrecyclings aus sekundären Quellen, gewichtet anhand der Massen des sekundären Kupfers aus Abschnitt 5.2.4, ergibt den durchschnittlichen KEA des Kupferrecyclings aus sekundären Quellen $(\overline{K E A})$. Diese Berechnung ist in Tabelle 5.3 vorgenommen. Im Durchschnitt benötigt das EoL-Recycling von Kupfer also $27 \mathrm{MJ} / \mathrm{kg}$ und verursacht 1,7 kg CO 2 eq-Emissionen. Letzterer Wert ist aus den in Kapitel C.1 des Appendix III des elektronischen Zusatzmaterials enthaltenen Modellergebnissen 
errechnet. Das EoL-Recycling von Kupfer benötigt somit im Durchschnitt lediglich $51 \%$ des KEA der aktuellen Primärgewinnung und verursacht $59 \%$ weniger $\mathrm{CO}_{2}$ eq-Emissionen.

Tabelle 5.3 Durchschnittlicher KEA des EoL-Kupferrecyclings

\begin{tabular}{l|l|l|l}
\hline Sekundäre Quelle $x_{i}$ & Sekundärkupfer [t] & $\begin{array}{l}K E A_{S}\left(x_{i}\right) \\
{[\mathrm{MJ} / \mathrm{kg} \mathrm{Cu}]}\end{array}$ & \begin{tabular}{l}
$($ Absoluter Anteil an) \\
\hline$E A_{s}[\mathrm{MJ} / \mathrm{kg} \mathrm{Cu}]$
\end{tabular} \\
\hline Kabelschrott & 45.674 & 14 & $(4)$ \\
\hline Elektroaltgeräte & 56.405 & 19 & $(6)$ \\
\hline Altautos & 5.091 & 26 & $(1)$ \\
\hline Rostasche & 5.324 & 41,5 & $(1)$ \\
\hline Bau- und Abbruchabfälle & 55.748 & 45 & $(15)$ \\
\hline Summe & 168.242 & & 27 \\
\hline
\end{tabular}

Der Vergleich der $K E A_{S}\left(x_{i}\right)$ mit dem KEA der primären Gewinnung von $51 \mathrm{MJ} / \mathrm{kg} \mathrm{Cu}$, lässt bereits erkennen, dass sich das Recycling aus allen sekundären Kupferquellen lohnt und zu entsprechenden Ersparnissen führt. Dennoch kann das EoL-Recycling von Kupfer durchaus in den Bereich der Primärgewinnung kommen. Das zeigen die sekundären Quellen mit relativ geringen Kupferkonzentrationen, wie die Rostasche und die Bau- und Abbruchabfälle. Wie sich der KEA des Recyclings nach den methodischen Annahmen aus Abschnitt 4.4.2 zur EoL-RQ verhält, ist in Abbildung 5.7a veranschaulicht. Die unterschiedlichen $K E A_{S}\left(x_{i}\right)$ führen zu einem überproportionalen Wachstum des KEA des Kupferrecyclings mit zunehmender EoL-RQ.

Da alle $K E A_{S}\left(x_{i}\right)$ des Kupferrecyclings geringer ausfallen als der KEA der primären Gewinnung, führt das EoL-Kupferrecycling, wie es aktuell praktiziert wird, zu einer Minderung des KEA und der $\mathrm{CO}_{2}$ eq-Emissionen der Kupferproduktion. Die aktuelle EoL-RQ von $49 \%$ entspricht damit auch der KEA-optimierten EoL-RQ bei den aktuellen Sammelquoten $S Q\left(x_{i}\right)$ und Recyclingeffizienzen $R E\left(x_{i}\right)_{j}$. Auf ein Jahr gerechnet werden bereits aktuell ca. $4 \mathrm{PJ}$ und $400.000 \mathrm{t} \mathrm{CO}_{2}$ eq-Emissionen durch das EoL-Recycling von Kupfer in Deutschland eingespart

Trifft die Annahme der zukünftigen Entwicklung der primären Kupfergewinnung hin zu einer vermehrten Gewinnung über die hydrometallurgische Route zu, werden sich die Ersparnisse, die durch das Kupferrecycling erzielt werden, voraussichtlich noch verstärken. Demnach würden sich auch in Zukunft keine Änderungen der energetisch optimalen EoL-RQ ergeben. Vielmehr wäre 


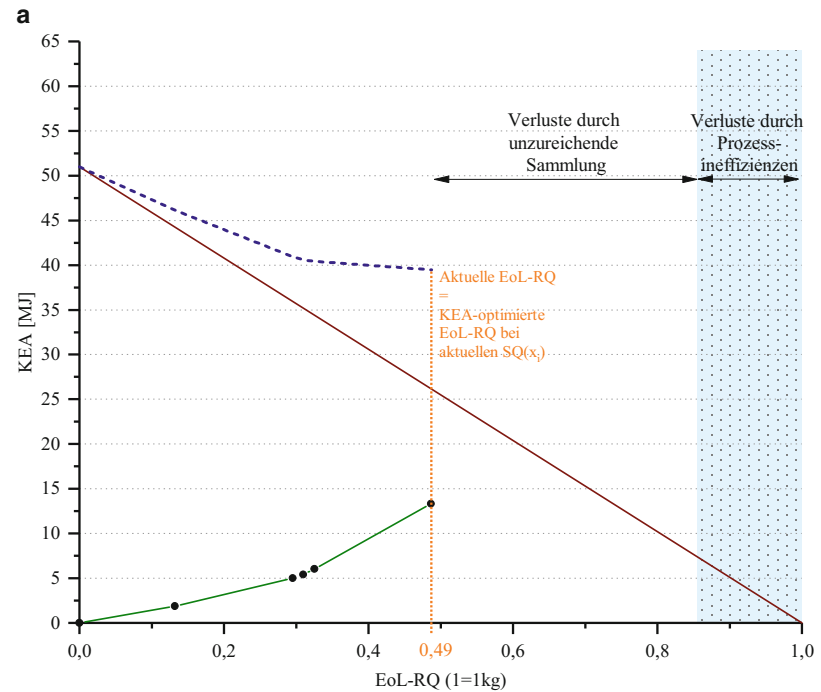

b

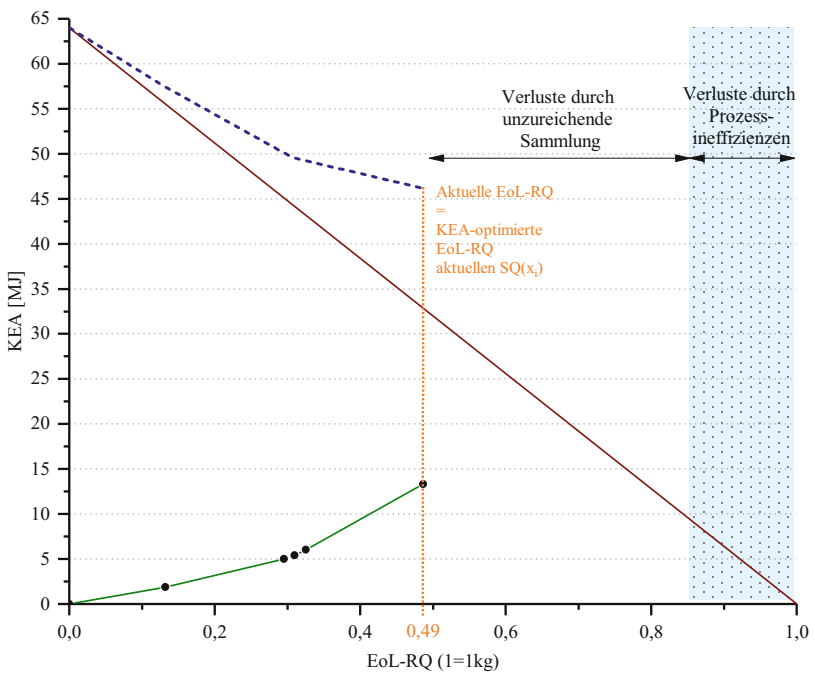

--- Total in MJ/kg - KEA des Primäranteils $\multimap-$ KEA des Recyclinganteils bei aktuellen SQ( $\left.\mathrm{x}_{\mathrm{i}}\right)$

Abbildung 5.7 KEA-optimierte EoL-RQ des Kupferrecyclings a der aktuellen Verhältnisse und $\mathbf{b}$ der abgeschätzten zukünftigen Verhältnisse der primären Kupfergewinnung 
davon auszugehen, dass die Ersparnisse durch das Recycling zunehmen, da die Aufwände der primären Gewinnung nach der vorgenommenen Abschätzung ansteigen. Die Auswertung ist in Abbildung 5.7b vorgenommen. Mögliche Änderungen in den Recyclingaufwänden - wie in Abschnitt 5.2.3.2 analysiert - sind aufgrund der hohen Unsicherheiten dieser Daten nicht berücksichtigt. Unter der getroffenen Annahme der zunehmenden hydrometallurgischen Aufbereitung kann jedoch festgehalten werden, dass zukünftig die $K E A_{S}\left(x_{i}\right)$ des Kupferrecyclings - selbst bei sekundären Quellen mit niedrigen Kupferkonzentrationen - um bis zu $50 \%$ ansteigen können und dennoch energieeffizienter wären, als die primäre Gewinnung.

\subsection{Szenarien-Analyse}

Die Auswertungen des vorherigen Abschnitts zeigen, dass das Recycling von Kupfer, wie es derzeit praktiziert wird, eine Reduktion der KEA und THGE bewirkt. Gleichzeitig bestehen noch große Potenziale für weitere Erhöhungen der EoL-RQ durch Verbesserungen der Sammelquoten $S Q\left(x_{i}\right)$. Das zeigt die Massenbilanz in Abschnitt 5.2.4. Aber auch Verbesserungen der Recyclingeffizienzen $R E\left(x_{i}\right)$ führen zu einer Steigerung der EoL-RQ. Wie sich letzteres auf die KEA-optimierte EoL-RQ auswirkt, kann wie in Abschnitt 4.4.2 erläutert, in der vorliegenden Arbeit nicht quantitativ untersucht werden. Es ist jedoch davon auszugehen, dass Verbesserungen der $R E\left(x_{i}\right)$ zu Anstiegen in den $K E A_{S}\left(x_{i}\right)$ führen und damit die energetisch optimale EoL-RQ entscheidend beeinflussen.

In Abschnitt 4.4.2 ist diskutiert, dass eine Erhöhung der Sammelquoten der sekundären Quellen $S Q\left(x_{i}\right)$ im Grunde nicht in höheren KEA pro Masseneinheit Kupfer $\left(K E A_{S}\left(x_{i}\right)\right)$ resultieren. Das ist darauf zurückzuführen, dass die durchschnittliche Transportdistanz, die zu den Sammelstellen zurückgelegt werden muss, für jedes Einzugsgebiet konstant ist. Erhöhungen der Sammelquoten haben somit keinen Einfluss auf die Transportdistanz. Diese Annahme gilt allerdings nur für die sekundärer Quellen $x_{i}$, deren Sammlung ausschließlich auf tatsächliche Transporte zurückzuführen ist. Für den selektiven Rückbau der Bauund Abbruchabfälle gilt diese Annahme nicht, da es sich hierbei um keine reine Sammlung im Sinne von Transporten, sondern vielmehr um eine mechanische Aufbereitung handelt.

Wie sich die KEA des Kupferrecyclings mit zunehmender EoL-RQ unter der Annahme der vollständigen Sammelquoten ohne Mehraufwände verhalten, ist in Abbildung 5.8a veranschaulicht. Die diskreten Datenpunkte der sekundären Quellen verschieben sich entsprechend der zunehmenden Anteile an der EoL-RQ nach rechts und entsprechend der Skalierung der $K E A_{S}\left(x_{i}\right)$ anhand der Anteile an der 

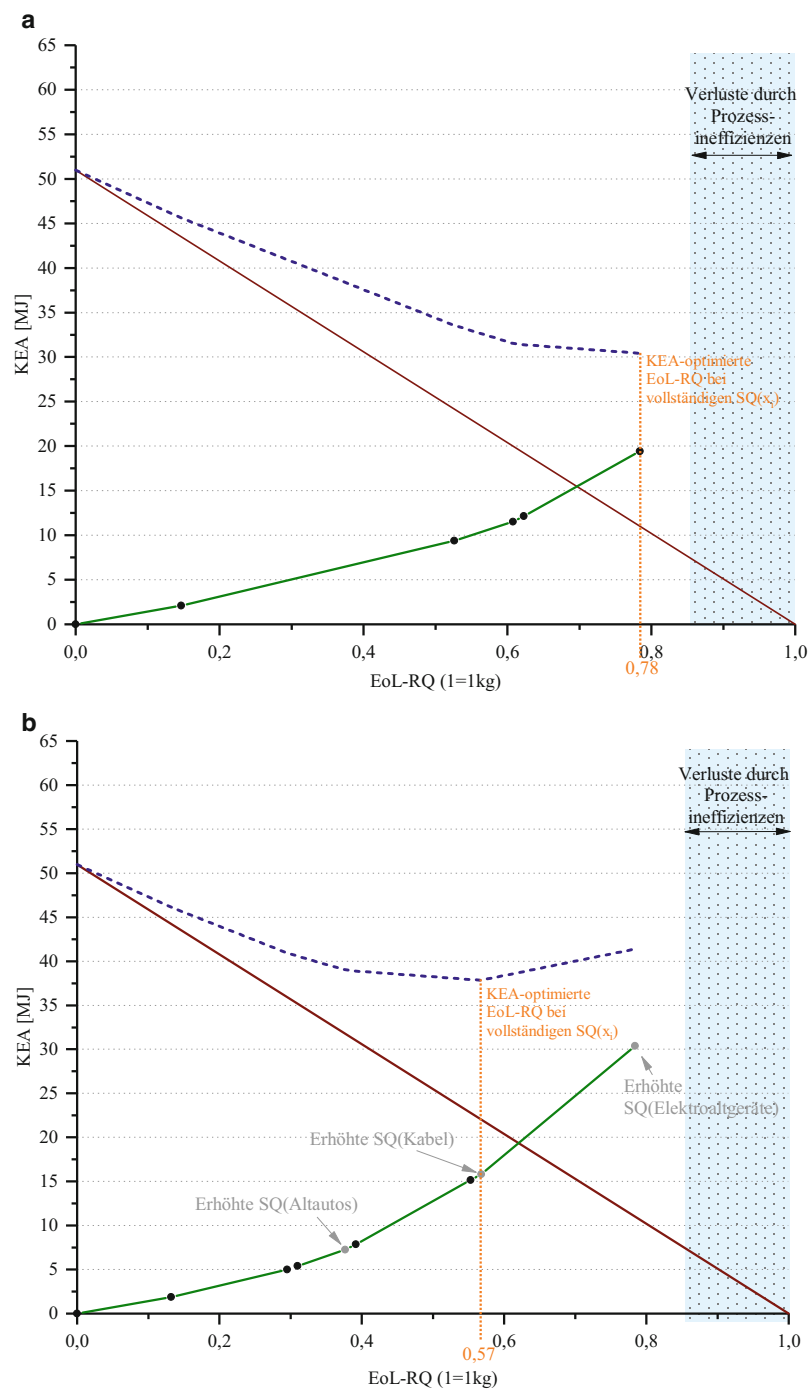

- - - Total in MJ/kg — KEA des Primäranteils $\longrightarrow$ KEA des Recyclinganteils bei vollständigen $\mathrm{SQ}\left(\mathrm{x}_{\mathrm{i}}\right)$

Abbildung 5.8 KEA-optimierte EoL-RQ des Kupferrecyclings bei vollständigen Sammelquoten und a konstanten Sammelaufwänden pro $\mathrm{kg} \mathrm{Cu}$ bzw. b variablen Sammelaufwänden pro kg Cu (5 \% Auslastung der Transportkapazitäten, ökonomische Allokation) 
EoL-RQ nach oben. Bei diesem Szenario verschiebt sich die energetisch optimale EoL-RQ auf $78 \%$.

Bei einer Steigerung der Sammelquoten $S Q\left(x_{i}\right)$ würden also Transporte vorgenommen werden, die bei aktuellen $S Q\left(x_{i}\right)$ nicht stattfinden. Bei (annähernd) vollständigen Sammelquoten $S Q\left(x_{i}\right) \rightarrow 1$ müssen auch geringste Mengen (z. B. das typische Beispiel vom Handy in der Schublade) in eine Sammlung gegeben werden. Das muss nicht zwangsläufig als Begleiteffekt eines ohnehin stattfindenden Transportes geschehen. Die Aufwände dieser Transporte können somit von den Aufwänden der für die aktuellen $S Q\left(x_{i}\right)$ stattfindenden Transporte abweichen. Um zu analysieren, wie stark die Transportaufwände variieren können und wie sich diese Änderungen auf die KEA-optimierte EoL-RQ auswirken, ist ein weiteres Szenario angenommen, bei dem die Auslastung der Kapazitäten der zusätzlich vorgenommenen Transporte auf $5 \%$ reduziert wird. ${ }^{3}$ Zudem sind für diese Transporte ökonomische Allokationen unterstellt. Sammlungen durch den Endkonsumenten, wie sie aktuell stattfinden, sind als Abfallentsorgungsmaßnahme eingestuft und daher nach den Massen alloziert (siehe Abschnitt 4.3.4). Den zusätzlich stattfindenden Transporten ist in diesem Szenario allerdings keine Entsorgungsnotwendigkeit zugeschrieben, da sie auch nach dem aktuellen Status Quo keiner notwendigen Entsorgung bedürfen bzw. über andere Wege, wie z. B. (illegale) Exporte eine Entsorgung fahren. Die Analyse ist in Abbildung 5.8b veranschaulicht. Da sich die $K E A_{S}\left(x_{i}\right)$ der sekundären Quellen, deren Sammelquoten $S Q\left(x_{i}\right)$ Verbesserungen unterstellt werden, ${ }^{4}$ bei aktuellen $S Q\left(x_{i}\right)$ und vollständigen $S Q\left(x_{i}\right)$ im angenommenen Szenario unterscheiden, sind sie als gesonderte diskrete Datenpunkte dargestellt. Der $K E A_{S}\left(x_{i}\right)$ der zusätzlich gesammelten Elektroaltgeräte übersteigt in diesem Szenario den KEA der primären Kupferproduktion. Die energetisch optimale EoL-RQ liegt bei $57 \%$.

Die zwei erstellten Szenarien zeigen, dass durch verbesserte Sammelquoten noch deutliche Potenziale für weitere Reduktionen der KEA und THGE existieren. Insbesondere die Sammelaufwände der Elektroaltgeräte können jedoch je nach Auslastung der Transportkapazitäten und Allokationsmethode stark schwanken und gegebenenfalls zu negativen Trade-offs führen. Da es sich hierbei um

\footnotetext{
${ }^{3}$ Die Änderungen der Transportkapazitäten gelten nicht für Altautos, da diese selbst Transportmittel und-gegenstand darstellen.

${ }^{4}$ Betrifft Kabelschrott, Elektroaltgeräte und Altautos. Bau- und Abbruchabfälle sind aufgrund der Sammlung durch selektiven Rückbau ausgenommen. Für Rostasche aus Siedlungsabfällen ist aufgrund der gesetzlichen Verpflichtung zur Aufbereitung bereits eine $S Q\left(x_{i}\right)$ von $100 \%$ angenommen (siehe Abschnitt B.1.3.1 in Appendix II des elektronischen Zusatzmaterials).
} 
Sammlungen durch Endkonsumenten handelt, sind weiterführende sozialwissenschaftliche Forschungen empfehlenswert, um genauere Berechnungen zu den Sammelaufwänden vornehmen zu können.

\subsubsection{Fazit zum Kupferrecycling}

Die Auswertungen der Ergebnisse des Recyclingmodells zeigen, dass die Kupferkonzentrationen der sekundären Quellen die $K E A_{S}\left(x_{i}\right)$ des Kupferrecyclings wesentlich beeinflussen. Dennoch benötigt das Kupferrecycling aus sekundären Quellen, die eine geringere Kupferkonzentration aufweisen als die primären Quellen (Rostasche, Bau- und Abbruchabfälle), einen geringeren KEA als die Primärgewinnung. Das ist u. a. auf Allokationseffekte zurückzuführen. Des Weiteren zeigen die Auswertungen des erstellten Recyclingmodells, dass das Kupferrecycling aus sekundären Quellen mit geringeren Konzentrationen tendenziell einen geringeren KEA pro Masseneinheit durchgesetztes Material $\left(K E A_{D}\left(x_{i}\right)\right)$ aufweist. Das ist zum einen auf die geringere Komplexität dieser sekundären Quellen und zum anderen auf die geringeren Recyclingeffizienzen ihrer mechanischen Aufbereitungen zurückzuführen.

Die Optimierung der EoL-RQ des Kupferrecyclings nach den energetischen Aufwänden zeigt, dass die aktuell erzielte EoL-RQ von $49 \%$ unter den gegebenen Bedingungen der Sammelquoten und Recyclingeffizienzen bereits optimal ist. Alle relevanten verfügbaren sekundären Kupferquellen werden aktuell mit energetischen Ersparnissen recycelt. Deutliche Optimierungspotenziale existieren insbesondere noch durch Verbesserungen der Sammelquoten. Auch zukünftig wird das EoL-Recycling von Kupfer aller Voraussicht nach energetisch vorteilhaft sein, sofern der angenommene Umschwung auf einen vermehrten Einsatz der aufwandsintensiveren hydrometallurgischen Route der primären Gewinnung zu verzeichnen sein wird. Die Auswertungen zeigen allerdings auch, dass die energetischen Aufwände des Kupferrecyclings durchaus in den Wertebereich der primären Kupfergewinnung kommen können. So sind die $K E A_{S}\left(x_{i}\right)$, die für das Recycling aus Rostasche und Bau- und Abbruchabfällen benötigt werden, nur marginal geringer als die der primären Gewinnung. Steigerungen der EoL-RQ dieser beiden sekundären Quellen durch erhöhte Energieeinsätze oder steigende Energiebedarfe durch Änderungen in deren Kupferkonzentrationen können also durchaus zu negativen Effekten führen.

Die Ergebnisse des Kupferrecyclingmodells und die damit verbundenen Auswertungen sind mit gewissen Unsicherheiten behaftet und daher nicht als definitive Ergebnisse zu verstehen, sondern vielmehr als erste richtungsweisende 
Abschätzungen. Das Recycling von Kupfer aus sekundären Quellen ist stark mit dem Recycling weiterer Metalle aus diesen sekundären Quellen verwoben. Das ist insbesondere auf die Funktion des Kupfers als Trägermetall zurückzuführen (siehe Abschnitt 5.2.3.1). Sollte Kupfer also nicht aus einer sekundären Quelle recycelt werden, so kann das negative Auswirkungen auf das Recycling zahlreicher weiterer Metalle haben, die ebenfalls in dieser sekundären Quelle enthalten sind. Auf diese Unsicherheiten ist ausführlich in Abschnitt 7.3 eingegangen.

\subsection{Tantal}

\subsubsection{Die Bedeutung von Tantal}

Tantal ist der Menschheit erst seit einer relativ kurzen Zeit bekannt. Die erste Entdeckung dieses Metalls ist auf das Jahr 1802 datiert (Wollaston 1809). Zum ersten Mal in verhältnismäßig reiner Form wurde es jedoch erst über 100 Jahre später, zu Beginn des 20. Jahrhunderts hergestellt (TIC 2018). Tantal ist der Gruppe der Refraktärmetalle zugeordnet, deren Besonderheit ihre sehr widerstandsfähigen und robusten Eigenschaften sind. Neben der hohen Duktilität, Härte und Dichte ist Tantal auch aufgrund des sehr hohen Schmelzpunktes von $2.996{ }^{\circ} \mathrm{C}$ ein begehrtes und vielseitig einsetzbares Technologiemetall. Das heutige Hauptein-

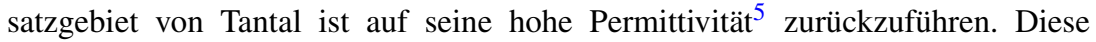
Eigenschaft prädestiniert Tantal für einen Einsatz als Anodenmaterial in Kondensatoren. Aufgrund dieses Einsatzgebietes ist Tantal heute in nahezu allen elektronischen Geräten verbaut (Schulenburg et al. 2017; Both 2016).

Im Jahr 2016 wurden weltweit 1.730 t Tantal gefördert. Davon sind über $60 \%$ auf den Kleinbergbau zurückzuführen (Damm 2018). Nach einer aktuellen Prognose wird im Jahr 2035 das 1,6-fache dieser Menge benötigt, um lediglich die zwei am stärksten wachsenden Anwendungsfelder - Superlegierungen und Kondensatoren - abdecken zu können (Marscheider-Weidemann et al. 2016). Eben diese zunehmende wirtschaftliche Bedeutung ist der wesentliche Grund, warum Tantal im Jahr 2017 wieder in die Liste der als kritisch eingestuften Rohstoffe der Europäischen Union aufgenommen wurde (EC 2017b). Das Versorgungsrisiko ist in dieser Kritikalitätsstudie als moderat eingestuft, trotz des hohen Anteils an artisanalem Bergbau, keinen innereuropäischen primären Tantalvorkommen und einer EoL-RQ von unter einem Prozent (Graedel et al. 2011). Diese niedrige EoL-RQ

\footnotetext{
${ }^{5}$ Die Permettivität, auch dielektrische Leitfähigkeit genannt, gibt die Durchlässigkeit eines Materials für elektrische Felder an.
} 
kann zu weiten Teilen durch die Hauptanwendung von Tantal in Kondensatoren erklärt werden, die in sehr geringen Mengen in einer Vielzahl an Produkten vorkommen, was die Umsetzung eines Recyclings erheblich erschwert. Außerdem ist Tantal zu lediglich 0,9 ppm in der Erdkruste (Rudnick und Gao 2004) und zu durchschnittlich 0,02\% in Minen (aktuelle primäre Quellen) vorhanden (Mackey und Simandl 2015) und ist somit auch primärseitig sehr niedrig konzentriert. Die Gewinnung von Tantal unterliegt damit primär- und sekundärseitig einem signifikanten Konzentrationsproblem. Eine genaue Analyse der KEA des Recyclings und ein Vergleich mit dem KEA der primären Gewinnung ist vor diesem Hintergrund von besonderer Bedeutung und kann wertvollen Aufschluss für Verbesserungspotenziale in der Tantalbereitstellung liefern.

\subsubsection{Sekundäre Tantalquellen}

Tantal kommt in sechs Hauptanwendungen - Kondensatoren, Legierungsadditiven, Tantaloxiden, Sputtertargets, Tantalpulver und Tantalkarbiden - zum Einsatz, die wiederum in zahlreichen verschiedenen Produkten benötigt werden (Schulenburg et al. 2017; Nassar 2017). Der mengenmäßig größte Anteil der Weltjahresproduktion fließt als hochreines Tantalpulver in die Herstellung von Kondensatoren. Diese werden auf Leiterplatten zur Speicherung elektrischer Ladung benötigt (Freeman 2018; Fitzpatrick et al. 2015) und finden daher hauptsächlich im Bereich der Informations- und Kommunikationstechnologie (IKT) und mittlerweile auch im Automobilbereich Anwendung (Deetman et al. 2017; Nassar 2017). Als Legierungsadditiv wird Tantal hauptsächlich in der Luftfahrt eingesetzt. Aufgrund dessen hoher Temperaturbeständigkeit findet der Einsatz insbesondere in Turbinen statt. Tantaloxide werden primär für optische Gläser, wie z. B. Linsen benötigt. Sputtertargets aus Tantal werden hauptsächlich in der Halbleiterindustrie eingesetzt, um Leiterplatten mit einem dünnen Tantalfilm zu beschichten, der als Barrierematerial dient. So werden Interdiffusionen in den stark kupferhaltigen Leiterplatten verhindert (Gilman 2010; Fitzpatrick et al. 2015). Ein weiteres Anwendungsfeld der Sputtertargets ist das Beschichten von Flachbildschirmen (Nassar 2017). Tantalkarbide kommen aufgrund ihrer Härteeigenschaften in Schneid- und Bohrwerkzeugen zum Einsatz. Die Einsatzgebiete von Tantalpulver sind überaus vielzählig und reichen von korrosionsbeständigen Beschichtungen von Schrauben über militärische Anwendungen in Projektilen bis zu Beschichtungen von Containern für nukleare Abfälle (Schulenburg et al. 2017; Nassar 2017). Abbildung 5.9 zeigt eine Abschätzung der prozentualen Aufteilung der globalen Tantalproduktion nach den sechs Hauptanwendungsgebieten. 


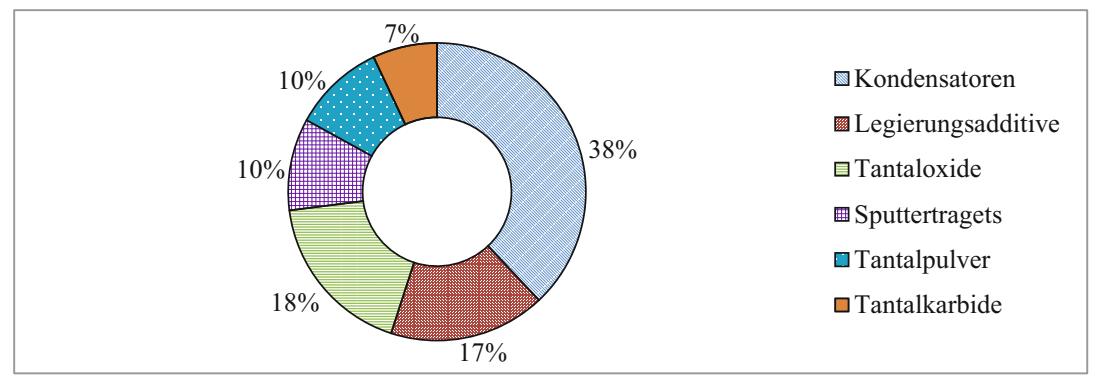

Abbildung 5.9 Hauptanwendungsgebiete Tantal (2015) (Schulenburg et al. 2017)

Die sechs Hauptanwendungen von Tantal fallen nach der Nutzungsphase als unterschiedlichste sekundäre Tantalquellen an. Bei der Identifikation der sekundären Quellen sind einige Besonderheiten zu beachten, die nachfolgend erläutert sind. Sputtertargets geben durch den Beschichtungsprozess Material an das zu beschichtende Objekt ab. Das Tantal, das nun auf den Leiterplatten bzw. den Bildschirmen verteilt ist, kann bis dato nicht rückgewonnen werden (Ciacci et al. 2015). Es sind derzeit auch keine Forschungen dazu aktiv. In diesem Fall kann von Dissipation gesprochen werden. Ein Recycling ist hier nur für die Reste der Sputtertargets nach deren Nutzung möglich, die zwischen 10 und $70 \%$ des Ausgangsgewichtes betragen (Marwede und Reller 2014; Nassar 2017). Da in Deutschland in keinem nennenswerten Umfang Leiterplatten oder Bildschirme produziert werden, fallen auch keine Sputtertargetreste an, die einem Recycling zugeführt werden könnten. Diese potenzielle sekundäre Quelle ist damit von den weiterführenden Untersuchungen ausgenommen. Auch die Anwendungsfelder des Tantalpulvers sind nicht weiterführend betrachtet. Die daraus resultierenden sekundären Quellen sind zum einen sehr unterschiedlich und zum anderen teils dissipativer Natur (siehe Beispiele oben). Zudem machen die zahlreichen Anwendungsfelder des Tantalpulvers jeweils nur sehr geringe Anteile an der verfügbaren Gesamtmasse des Tantals in sekundären Quellen aus. Denn bereits die Summe aller eingesetzten Tantalpulver betragen lediglich $10 \%$ am gesamten Tantalbedarf. Nach Angaben von Ciacci et al. (2015) existieren derzeit ohnehin keine Recyclingmöglichkeiten bzw. Bemühungen, diese potenziellen sekundären Tantalquellen zu erschließen.

Die für die nachfolgenden Untersuchungen identifizierten sekundären Quellen sind Kondensatoren, Legierungen, Tantaloxide in optischen Gläsern und Tantalkarbide in Bohr- und Schneidwerkzeugen. Tantalkondensatoren sind in teils stark 
unterschiedlichen Produkten verbaut, die unterschiedliche Tantalkonzentrationen aufweisen und in unterschiedlichsten Massen anfallen (Deetman et al. 2017). Es ist also eine differenzierte Betrachtung dieser Produkte notwendig. Existierende Untersuchungen zu den Tantalgehalten in Elektrogeräten (bzw. Elektroaltgeräten) verweisen auf folgende Produkte in denen Tantalkondensatoren verbaut sind: Mobiltelefone, Tablets, Laptops, Desktop PCs, Server, Festplatten bzw. Hard Disk Drivers (HDD) und Flachbildschirme bzw. Flat Screen Monitore (FSM) (Ueberschaar et al. 2017a; Fitzpatrick et al. 2015; Chancerel et al. 2015). Eine weitere sekundäre Quelle, die Tantalkondensatoren enthält, sind Altautos bzw. deren elektronische Komponenten (Widmer et al. 2015). Alle sekundären Tantalquellen sowie deren Tantalkonzentrationen sind in Tabelle 5.4 zusammengefasst.

Tabelle 5.4 Sekundäre Tantalquellen und deren Tantalkonzentrationen (Datengrundlage in Kapitel B.1 des Appendix II des elektronischen Zusatzmaterials)

\begin{tabular}{l|l|l}
\hline \multicolumn{2}{l|}{ sekundäre Quelle } & $\omega_{T a}\left(x_{i}\right)[\mathrm{g} / \mathrm{g}]$ \\
\hline \multirow{3}{*}{ Kondensatoren } & Mobiltelefone & $1,19 \mathrm{E}-03$ \\
\cline { 2 - 3 } & Smartphones & $8,26 \mathrm{E}-05$ \\
\cline { 2 - 3 } & Tablets & $1,50 \mathrm{E}-04$ \\
\cline { 2 - 3 } & Notebooks & $8,13 \mathrm{E}-04$ \\
\cline { 2 - 3 } & Desktops & $3,90 \mathrm{E}-04$ \\
\cline { 2 - 3 } & Server & $7,08 \mathrm{E}-05$ \\
\cline { 2 - 3 } & HDD & $3,39 \mathrm{E}-04$ \\
\cline { 2 - 3 } & FSM & $4,38 \mathrm{E}-05$ \\
\cline { 2 - 3 } & Altautos ${ }^{\mathrm{a}}$ & $5,48 \mathrm{E}-06$ \\
\hline \multicolumn{2}{c}{ Karbide } & $4,00 \mathrm{E}-02$ \\
\hline \multicolumn{2}{c}{ Legierungen } & $4,80 \mathrm{E}-02$ \\
\hline
\end{tabular}

${ }^{a}$ Für Altautos ist die Konzentration in den elektronischen Komponenten angenommen, da diese Komponenten nach aktuellem Stand ohnehin aus Altautos demontiert werden (Kühn 2018). 


\subsubsection{Recyclingmodell}

\subsubsection{Recyclingverfahren und Prozessdaten}

Die sekundären Tantalquellen sind in ihren chemischen und physikalischen Zusammensetzungen so unterschiedlich, dass auch deren jeweilige mechanische und metallurgische Recyclingverfahren fundamental voneinander abweichen. Im Folgenden sind die Recyclingverfahren kurz zusammengefasst, um das erstellte Recyclingmodell und die darauf aufbauenden Auswertungen nachvollziehbar zu machen. Detailliertere Beschreibungen sowie die Erläuterungen und Referenzen zu den Prozessdatenerhebungen der Recyclingverfahren sind in Kapitel B.2 des Appendix II des elektronischen Zusatzmaterials gegeben.

Das Recycling von Tantal aus EoL-Kondensatoren befindet sich bis dato noch in der Forschung. Das angenommene Recyclingverfahren - siehe Abbildung 5.10 - setzt sich daher aus Prozessen im Pilot- bzw. Labormaßstab zusammen. Es sind die Prozesse mit den besten Prozessdaten ausgewählt. Für die metallurgische Aufbereitung konnte auf Prozessdaten des großindustriellen Maßstabes zurückgegriffen werden, da Kondensatoren, die als Produktionsabfälle anfallen, bereits recycelt werden (Schulenburg et al. 2017). Das Recycling von Tantal aus EoL-Kondensatoren benötigt eine umfangreiche und mehrstufige mechanische Aufbereitung, um die Kondensatoren von den Elektroaltgeräten bzw. deren Leiterplatten zu separieren. Die separierten Kondensatoren haben danach einen Tantalgehalt von ca. $50 \%$ (Ueberschaar et al. 2017a). Durch die mechanische Aufbereitung findet also bereits eine wesentliche Konzentrationserhöhung des Tantals statt. Die Kondensatoren werden in einem nachfolgenden Pyrolyseprozess von allen organischen Bestandteilen befreit. Weitere unerwünschte Komponenten werden über zusätzliche mechanische Aufbereitungsschritte entfernt. Über eine hydrometallurgische Aufbereitung wird, unter dem Zusatz von Chlor, aus der aufbereiteten Tantalfraktion reines Tantaloxid erzeugt. Eine finale Reduktion mit Magnesiumdampf reduziert das Oxid zu hochreinem Tantal.

Tantal, das in oxidischer Form in optischen Gläsern gebunden ist, kann über das sogenannte pyrometallurgische Syncon-Verfahren der H.C. Starck GmbH am Standort in Laufenburg und einer finalen Raffination am Standort in Goslar rückgewonnen werden (Schulenburg et al. 2017). Detaillierte Informationen zu diesem Verfahren sind Abschnitt B.2.1 in Appendix II des elektronischen Zusatzmaterials zu entnehmen. Das mengenmäßig größte Anwendungsgebiet der Tantaloxide sind mit fast $100 \%$ die optischen Linsen. Der Großteil ist davon auf Kameralinsen zurückzuführen (Deetman et al. 2017). In der Praxis werden derzeit lediglich Produktionsschrotte von optischen Gläsern einem Recycling zugeführt (Beckmann 2018). 


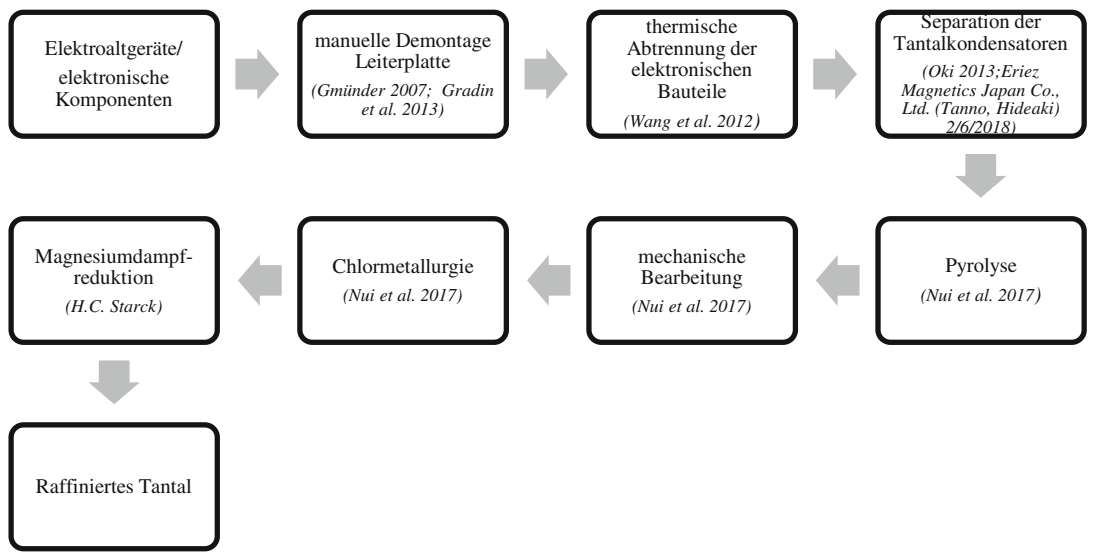

Abbildung 5.10 Verfahren des Tantalrecyclings aus EoL-Kondensatoren (Referenzen der Prozesse in Klammern)

Im Gegensatz zu Kondensatoren und optischen Gläsern findet das EoLRecycling von Tantal aus Legierungen und Karbiden in der Praxis bereits statt (Nassar 2017; Schulenburg et al. 2017). Allerdings wird das Tantalrecycling dieser beiden sekundären Quellen als Materialverbund vorgenommen, d. h. die einzelnen Materialien dieser sekundären Quellen werden nicht voneinander separiert, sondern im Verbund recycelt. Im Falle der Legierungen handelt es sich beim Recycling lediglich um einen Umschmelzprozess. Aufgrund der hohen Wertigkeit solcher tantalhaltigen Superlegierungen findet meist eine genaue Vorsortierung statt, sodass die Beimengung unerwünschter Legierungsbestandteile weitestgehend ausgeschlossen ist (Eckelman et al. 2014). Um Karbide zu recyceln wird in der Regel der Zink-Prozess angewandt (Kurylak et al. 2016b). Dabei wird unter Argon- oder Stickstoffschutzatmosphäre bei ca. $950{ }^{\circ} \mathrm{C}$ dem karbidischen Material Zink beigemengt, was zu einem chemischen Aufschluss des Bindemittels führt. Die stoffliche Zusammensetzung des Karbides ändert sich dabei nicht. Über eine Vakuumdestillation wird im Anschluss das Zink separiert und das Karbid kann erneut eingesetzt werden. Alle sekundärquellenspezifischen Prozessdaten der Modellbildung sind in der nachfolgenden Tabelle 5.5 enthalten.

Die Sammel- und Transportdistanzen des Tantalrecyclings sind, sofern möglich, basierend auf realen Standorten errechnet. Da Kondensatoren und optische Gläser bisher keinem EoL-Recycling zugeführt werden, sind bei deren Simulation der Sammel- und Transportlogistik Annahmen getroffen und Szenarien gerechnet. 
进

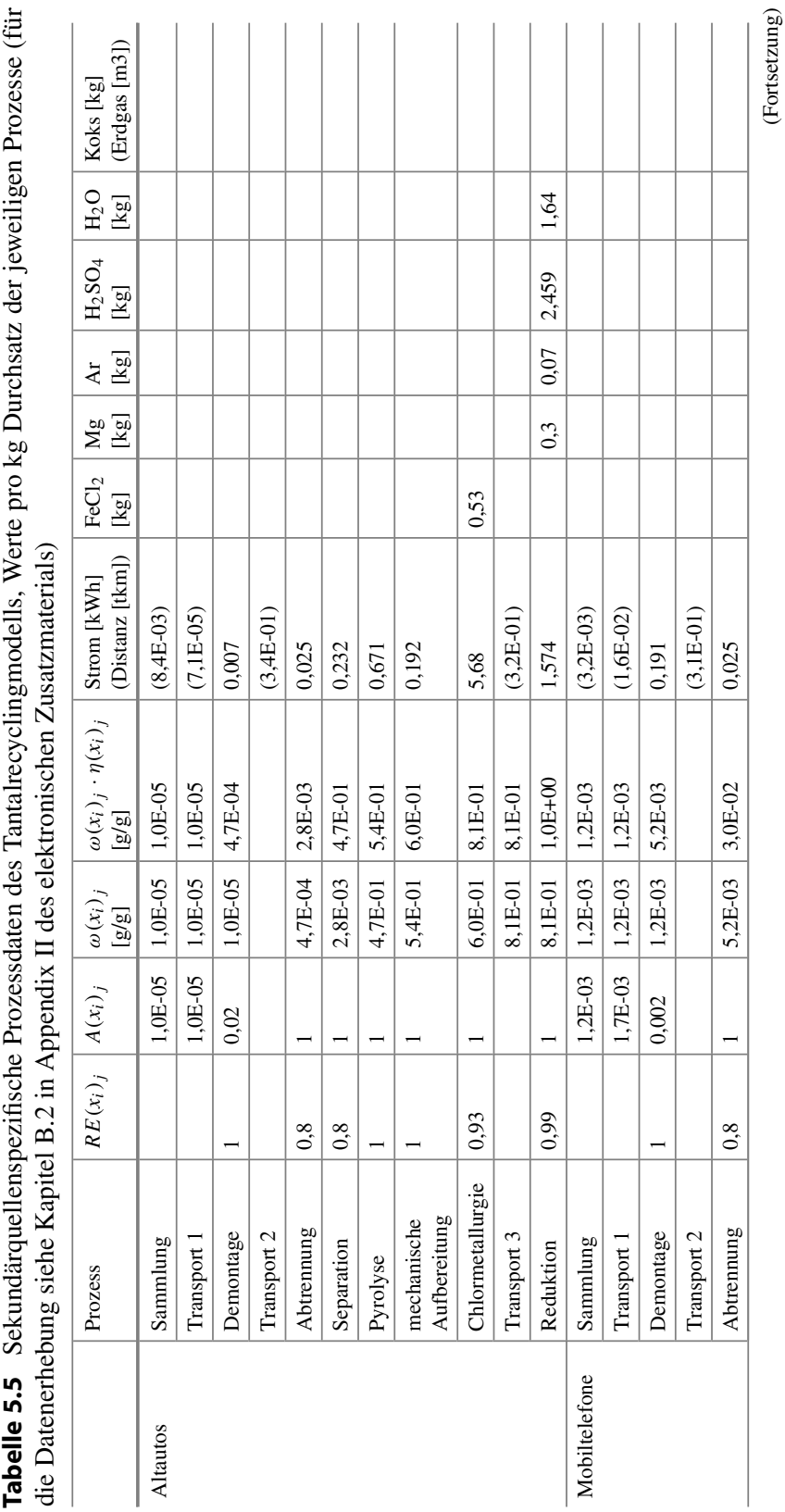




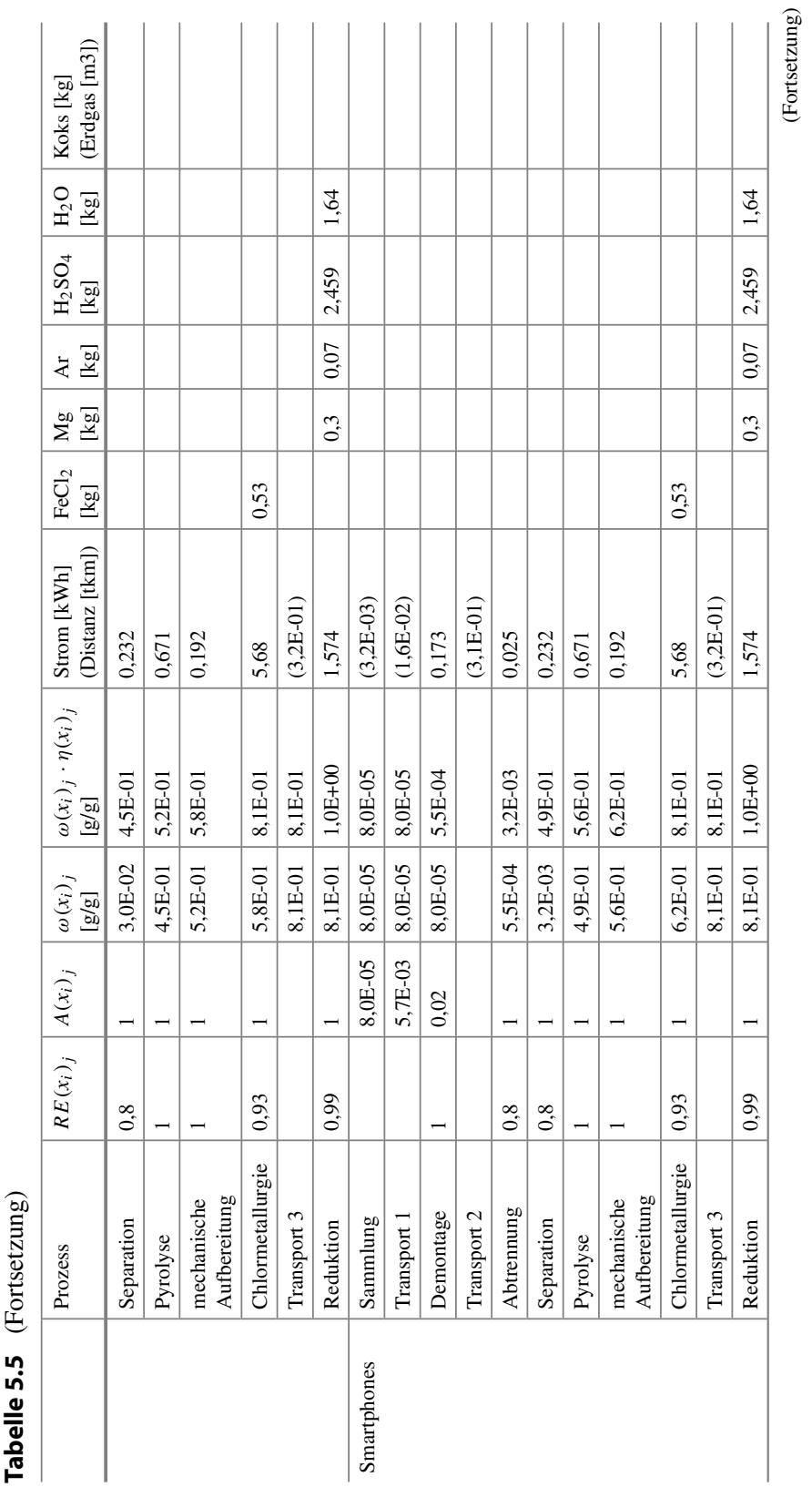




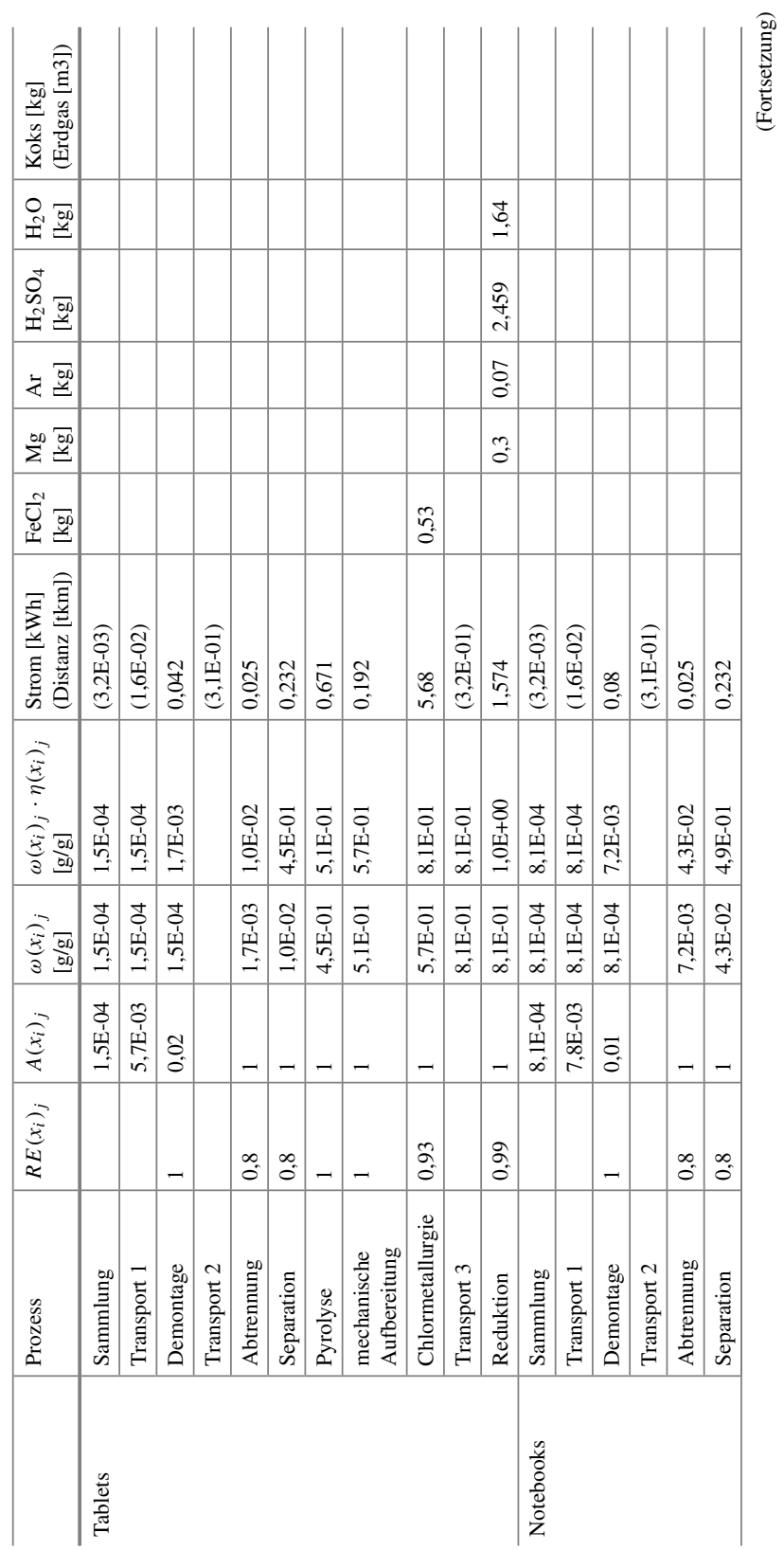




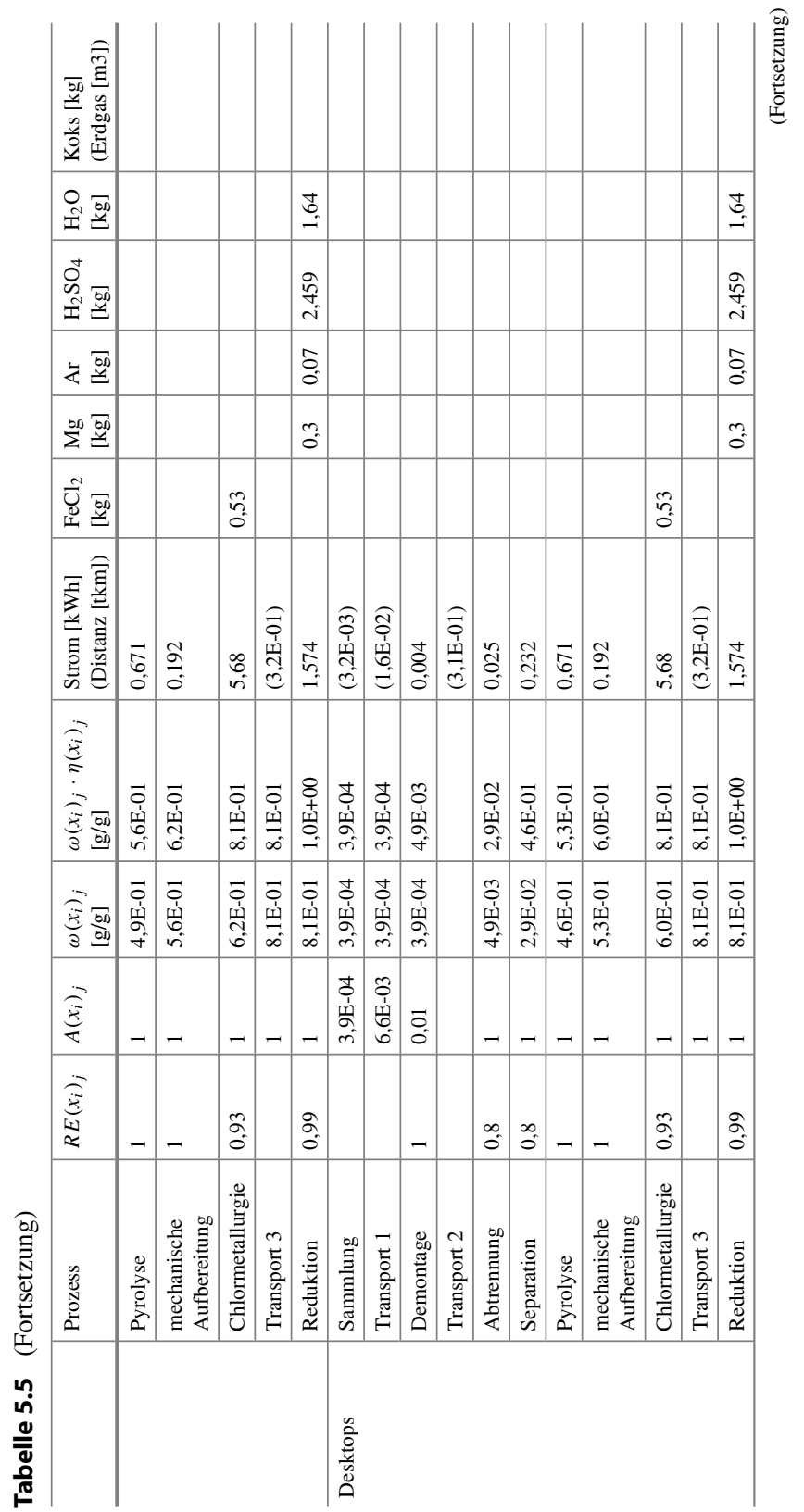




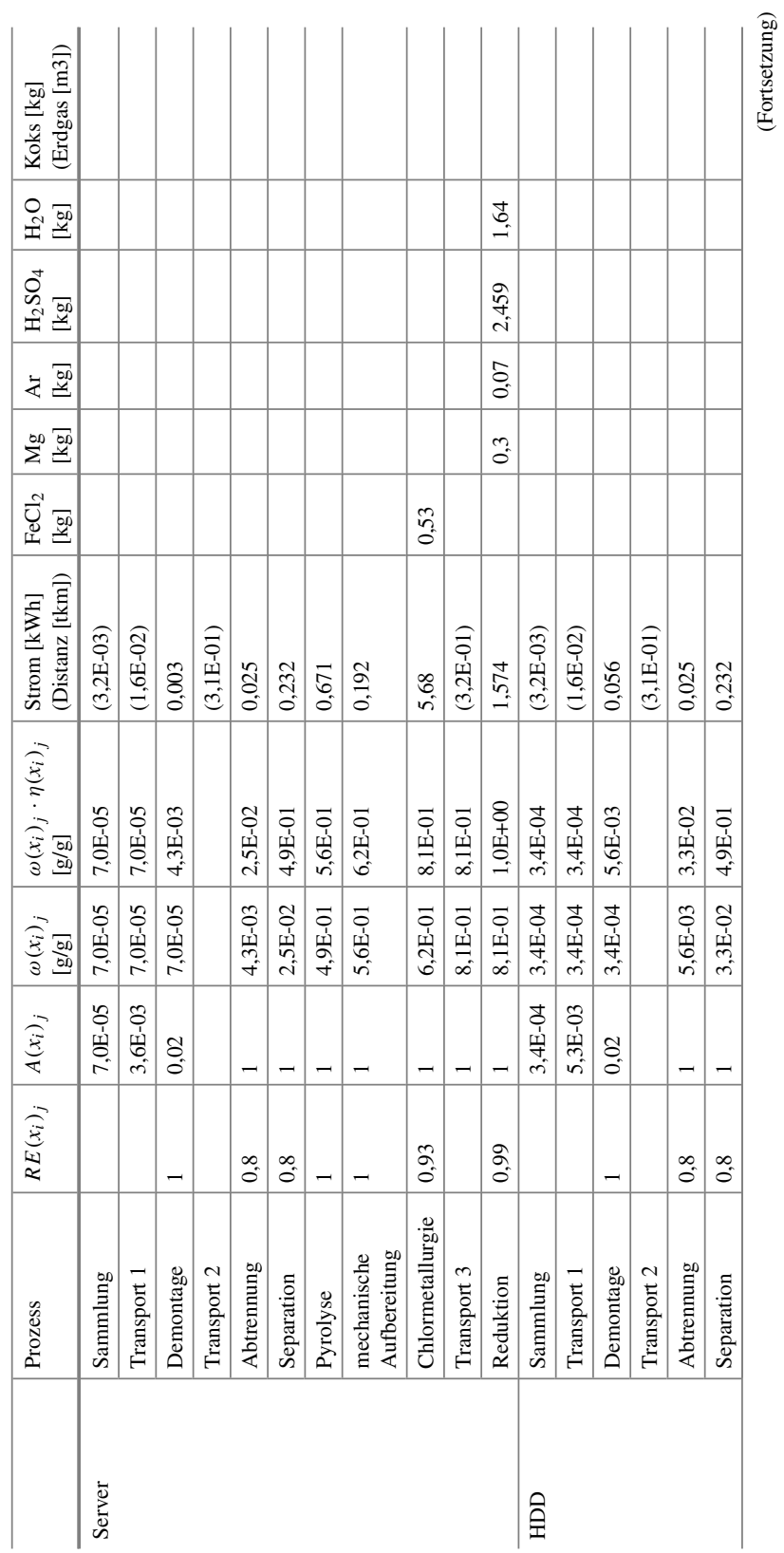




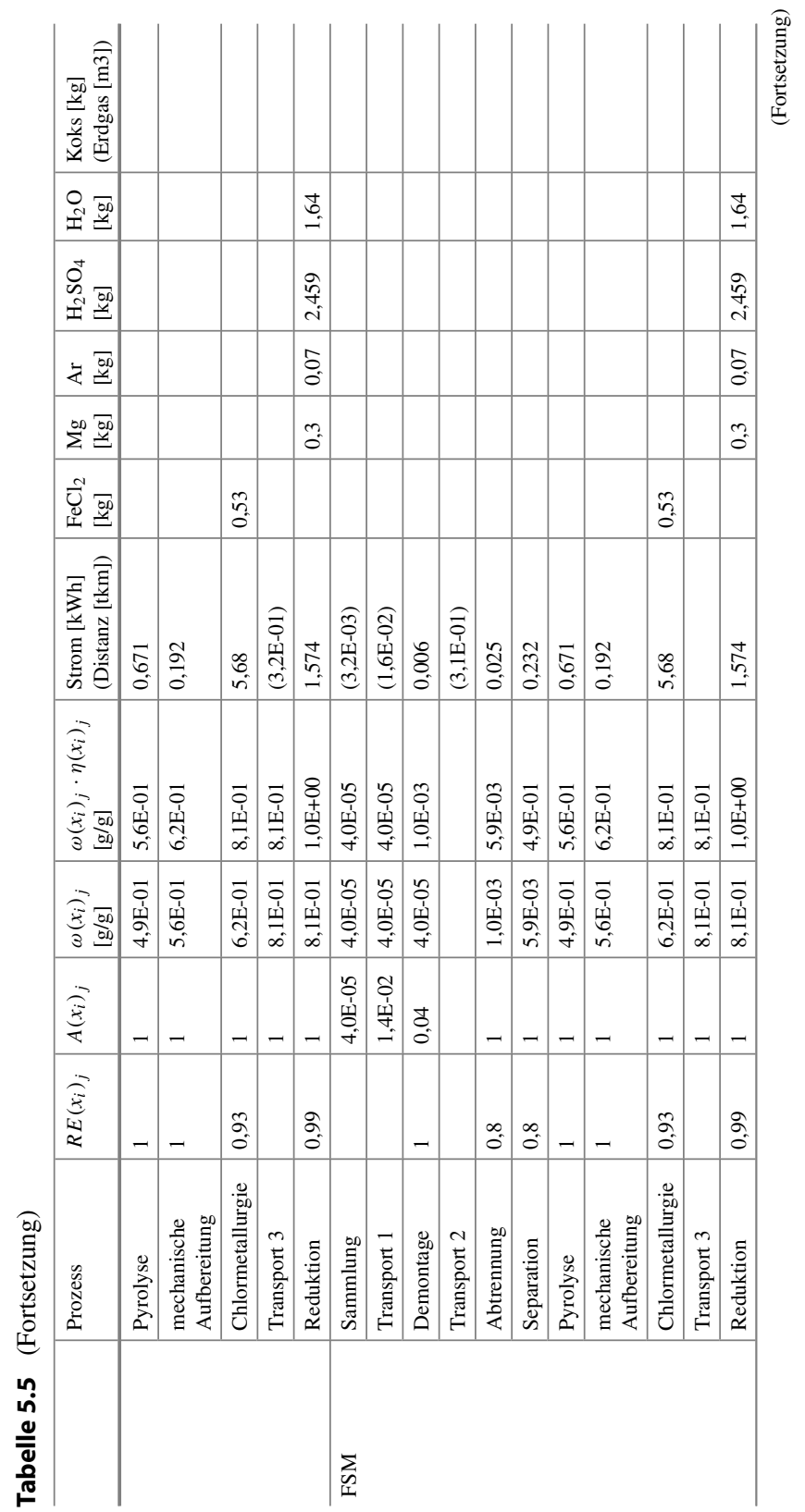




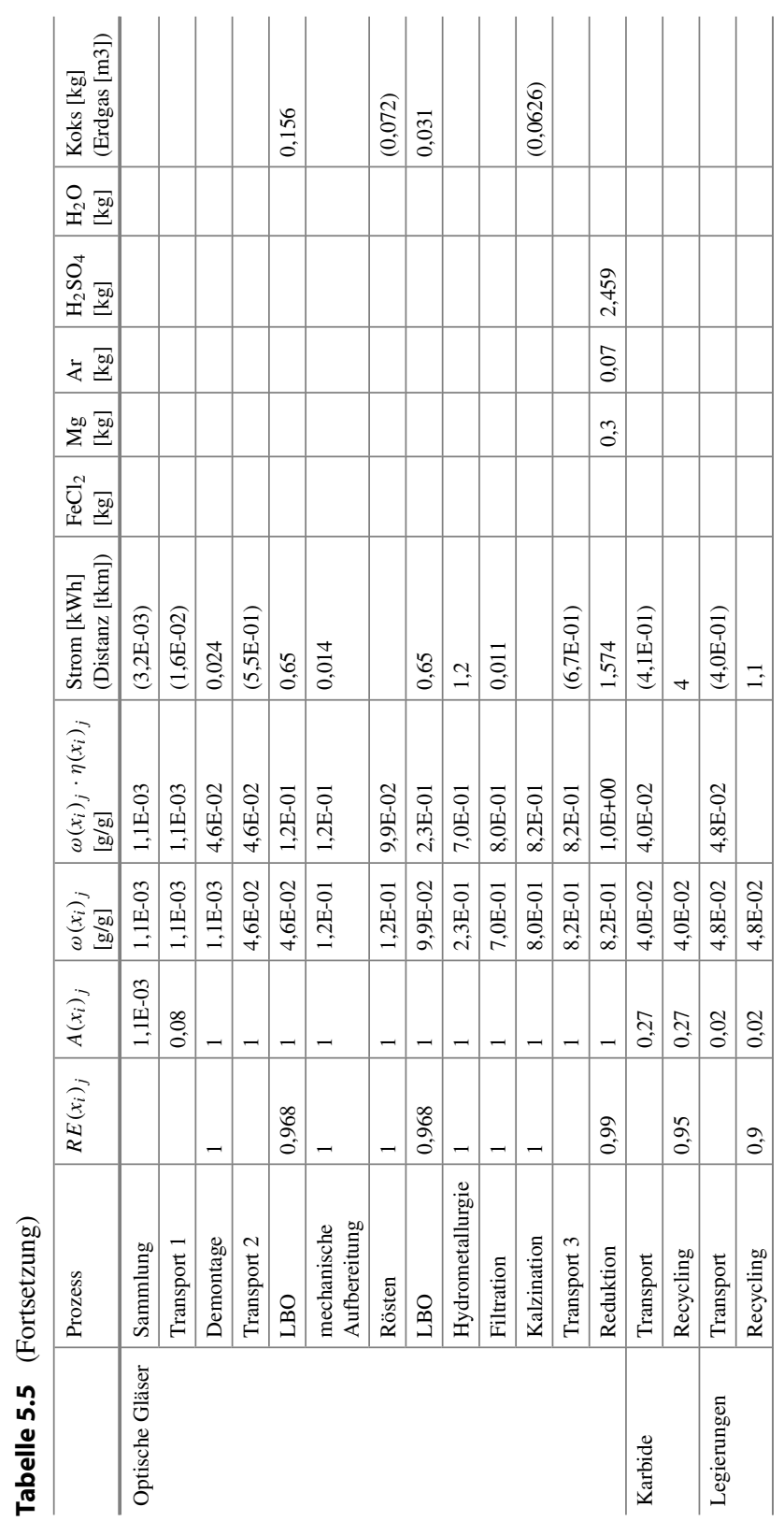


Kondensatoren und optische Gläser sind in Elektroaltgeräten und Altautos verbaut. Deren Sammeldistanzen entsprechen also denen der Elektroaltgeräte bzw. der Altautos. Auch die Distanzen des Transportes 1 zur weiterführenden Aufbereitungsanlage entsprechen den Distanzen dieser beiden sekundären Quellen. Am Ort der weiterführenden Aufbereitungsanlage ist die Demontage der Leiterplatten angenommen - was in der Praxis auch tatsächlich meist der Fall ist (Fröhlich 2018c). Nach der Demontage kann die weitere Aufbereitung nach zwei Szenarien erfolgen: (1) die Abtrennung der Kondensatoren von den demontierten Leiterplatten findet dezentral bei den weiterführenden Aufbereitungsanlagen statt, oder (2) die Abtrennung findet zentralisiert statt. Bei Szenario (2) muss weiter differenziert werden: (a) die zentrale Abtrennung wird an einem Ort vorgenommen, an dem die restliche Leiterplatte nicht aufbereitet werden kann, oder (b) an einem Ort, an dem die Aufbereitung der Leiterplatte ebenfalls realisiert werden kann. Beim weiterführenden Transport 2 des Szenarios (1) werden demnach nur die Kondensatoren transportiert. Im Szenario (2) werden die gesamten Leiterplatten transportiert. Im Szenario (2a) müssen die dabei anfallenden Aufwände des Transportes 2 vollständig Tantal zugerechnet werden, da keine Kuppelproduktion am Ort der zentralen Aufbereitung stattfindet. Im Szenario (2b) ist hingegen eine Allokation mit dem Faktor 0,01 für Tantal vorgenommen, was auf die hohe Wertigkeit der restlichen Leiterplatte zurückzuführen ist (die Ermittlung des Allokationsfaktors kann Abschnitt B.2.2.1.1 in Appendix II des elektronischen Zusatzmaterials entnommen werden). Diese Allokation ist notwendig, da der Transport 2 in Szenario (2b) neben dem Tantalrecycling auch dem Recycling der restlichen Leiterplatte dient.

Für alle drei Szenarien gelten die gleichen Transportdistanzen, sie unterscheiden sich damit lediglich in den zu transportierenden Massen und Allokationsfaktoren. Die Tantalkonzentrationen und Allokationsfaktoren des Transportes 2 sind somit auch nicht in Tabelle 5.4 mitaufgeführt. Sie können je nach Szenario den entsprechenden vor- und nachgelagerten Prozessen entnommen werden. Der Ort der zentralisierten Abtrennung der Kondensatoren (Szenario (2)) ist gleichzeitig als der Ort der weiteren Aufbereitung (Pyrolyse, mechanische Aufbereitung und Chlormetallurgie; auch als Prä-Metallurgie bezeichnet) angenommen. Da das EoL-Recycling von Kondensatoren nicht stattfindet, existiert auch kein realer Ort der weiterführenden Aufbereitung. Es ist daher ist ein fiktiver Standort festgelegt, welcher der durchschnittlichen Entfernung aller Punkte in Deutschland zum Standort der finalen Reduktion in Goslar entspricht.

Die Sammel- und Transportlogistik der optischen Gläser ist bis zur Demontage der Linsen aus den Kameras durch die Sammel- und Transportlogistik der Elektroaltgeräte abgedeckt. Der Transport 2 findet von den Aufbereitungsanlagen nach 
Laufenburg, der Transport 3 von Laufenburg nach Goslar statt. Die Transportlogistik der Legierungen und Karbide sind mit jeweils einer durchschnittlichen Transportdistanz vom Ort des Anfallens zur finalen Aufbereitung angenommen. Auch hierfür können weitere Informationen dem Abschnitt B.2.2.2 in Appendix II des elektronischen Zusatzmaterials entnommen werden.

\subsubsection{Modellergebnisse}

Das Umberto-Modell des EoL-Tantalrecyclings ist in Abbildung 5.11 dargestellt. Für diese Auswertung ist ein Mittelwert der in Abschnitt 5.3.3.1 beschriebenen Transportszenarien angenommen. Die Sankey-Darstellung quantifiziert die KEA der Prozesse. Je breiter der Pfeil, desto höher der KEA des Prozesses. Die Outputs sind auf jeweils $1 \mathrm{~kg}$ Sekundärtantal skaliert. Der genaue Aufbau eines solchen Umberto-Modells ist in Abschnitt 5.2.3 am Beispiel Kupfer erläutert. Vergleicht man das Modell des Tantalrecyclings mit dem des Kupferrecyclings, so fällt auf, dass sich das Recycling von Tantal aus den sekundären Quellen der Kondensatoren und optischen Gläsern weitaus komplexer gestaltet als das Recycling von Kupfer. Die Recyclingverfahren von Karbiden und Legierungen weisen moderate Komplexitäten auf.

Das Recycling von Tantal aus Kondensatoren ist zwischen den spezifischen sekundären Quellen (Mobiltelefone, Smartphones, Tablets, Notebooks, Desktops, Server, HDD, FSM und Altautos) differenziert. Alle diese sekundären Quellen durchlaufen zwar dieselben Recyclingprozesse, die Sankey-Darstellung gibt jedoch zu erkennen, dass diese Prozesse je nach sekundärer Quelle unterschiedlich hohe KEA benötigen. Diese Unterschiede sind unmittelbar auf die unterschiedlichen Tantalkonzentrationen und die unterschiedlichen Materialzusammensetzungen der sekundären Quellen zurückzuführen (siehe Tabelle 5.5). Letztere resultieren in unterschiedlichen Allokationsfaktoren. $\mathrm{Zu}$ Beginn der Recyclingkette sind die Tantalkonzentrationen in den sekundären Quellen noch relativ gering (siehe Tabelle 5.5). Durch Konzentrationseffekte fallen daher die KEA der Prozesse, die sich am Anfang der Recyclingkette befinden, verhältnismäßig hoch aus. Insbesondere die Demontage der Leiterplatten benötigt pro kg Tantal enorme KEA. Da dieser Prozess jedoch die Rückgewinnung zahlreicher weiterer Metalle unterstützt, ist auf Tantal nur ein Bruchteil des KEA alloziert. Gleiches gilt für die Sammlung, den Transport 1 und den Transport 2 des Szenarios (2b). Alle weiteren Recyclingschritte verfolgen ausschließlich das Ziel des Tantalrecyclings. Deren KEA sind demnach vollständig auf Tantal zugerechnet. ${ }^{6}$

\footnotetext{
${ }^{6}$ Sind dennoch Abflüsse der KEA in Outputstellen in Abbildung $5.11 \mathrm{zu}$ erkennen, ist das darauf zurückzuführen, dass die KEA zwar an früheren Prozessen alloziert sind, hier jedoch
} 


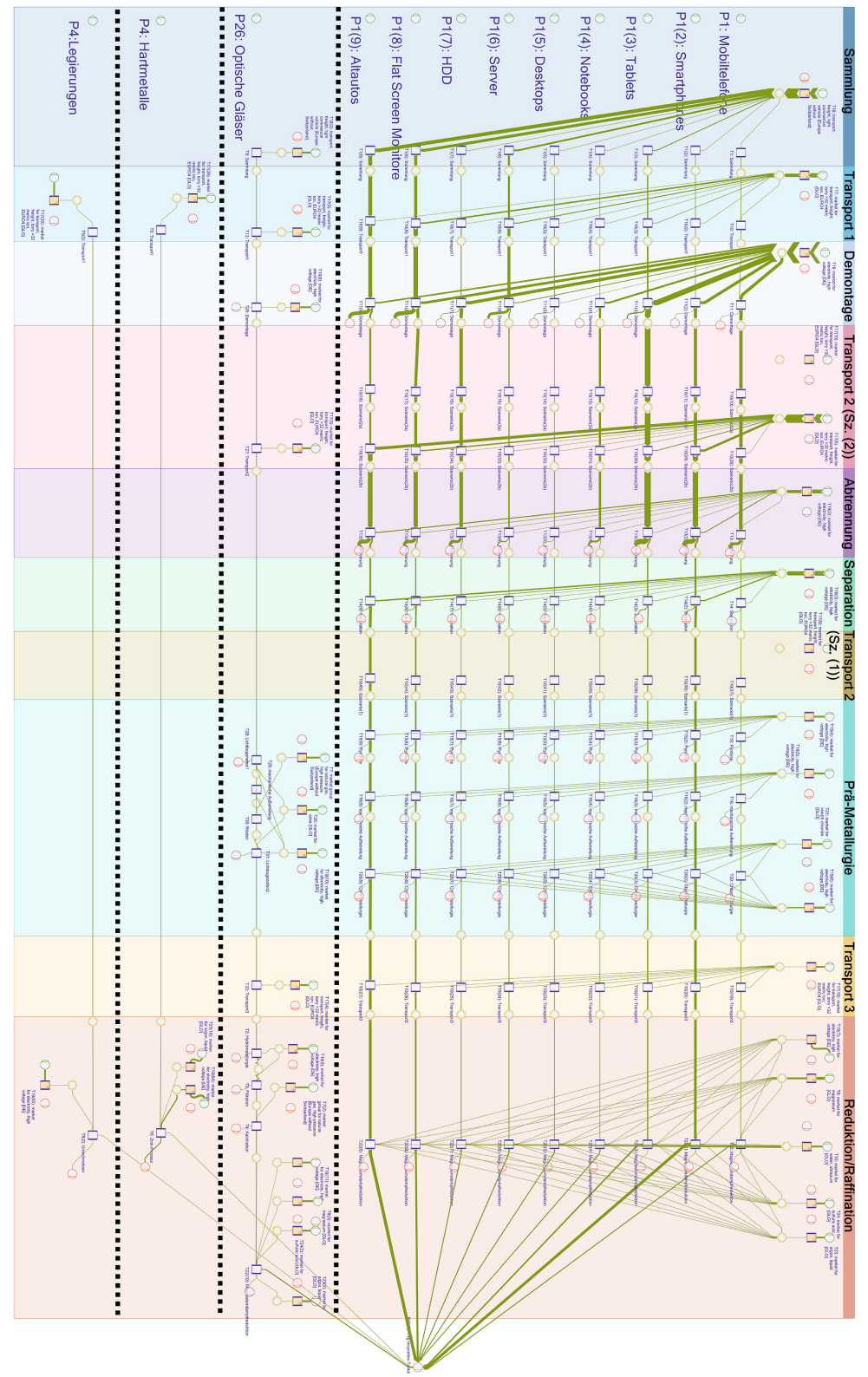

Abbildung 5.11 Umberto-Modell des Tantalrecyclingsystems (Sankey-Darstellung der KEA, alloziert) 
Die Auswertungen des Tantalrecyclingmodells nach den $K E A_{S}\left(x_{i}\right)$ sind in Abbildung 5.12 dargestellt. Es ist dabei zwischen den Szenarien (1) und (2a) - die als die beiden Extremszenarien des Transportes 2 verstanden werden können - sowie dem Mittelwert aus allen drei Szenarien unterschieden. Die Ergebnisse zeigen, dass der Transport wesentlichen Einfluss auf die KEA des Tantalrecyclings aus Kondensatoren haben kann. So weichen die $K E A_{S}\left(x_{i}\right)$ der beiden Extremszenarien um bis zu $65 \%$ ab, was ausschließlich auf die für den Transport 2 getroffenen Annahmen zurückzuführen ist. Um das Tantalrecycling aus Kondensatoren möglichst energieeffizient zu gestalten, sollte also das dafür notwendige Recyclingsystem so abgestimmt sein, dass Transporte, die dem Szenario (2a) gleichkommen, weitestgehend ausgeschlossen werden können. Um die nachfolgenden Analysen möglichst objektiv vornehmen zu können, sind für die Transportaufwände Mittelwerte, also eine Gleichgewichtung aller drei Transportszenarien, angenommen. Eine differenzierte tabellarische Auflistung der Modellergebnisse ist in Kapitel C.2 des Appendix III des elektronischen Zusatzmaterials enthalten.

Kondensatoren aus Altautos sind mit 3,7 GJ/kg Ta die sekundären Quellen, deren Tantalrecycling am meisten Energie benötigt, dicht gefolgt von Smartphones mit 3,4 GJ/kg Ta. Den geringsten $K E A_{S}\left(x_{i}\right)$ für das Tantalrecycling aus Kondensatoren benötigt die sekundäre Quelle der Notebooks. Über alle sekundären Quellen hinweg benötigt das Recycling von Karbiden und Legierungen am wenigsten Energie pro Masseneinheit Tantal.

Die KEA des Tantalrecyclings gehen, wie in Abbildung 5.11 und 5.12 gezeigt, je nach sekundärer Quelle stark auseinander. Diese hohe Bandbreite der $K E A_{S}\left(x_{i}\right)$ ist im Wesentlichen auf die unterschiedlichen Konzentrationen des Tantals in den sekundären Quellen zurückzuführen. Das zeigt die Korrelationsanalyse in Abbildung 5.13, die den Zusammenhang zwischen den $K E A_{S}\left(x_{i}\right)$ und den Tantalkonzentration über alle untersuchten sekundären Quellen quantifiziert. Mit einem Bestimmtheitsmaß von $\mathrm{R}^{2}=0,77$ weist der KEA des Recyclings von Tantal eine hohe Abhängigkeit von den Tantalkonzentrationen auf. Da die $K E A_{S}\left(x_{i}\right)$, wie auch die Konzentrationen der jeweiligen sekundären Quellen, um teils mehrere Größenordnungen auseinanderliegen, ist für Abbildung 5.13 eine doppellogarithmische Darstellung gewählt. Mit sinkender Konzentration folgen die KEA des Recyclings einem Potenzwachstum.

erst der physische Abfluss des Kuppelproduktes stattfindet. Ein Beispiel ist die Allokation der Demontageaufwände der Leiterplatten: ein Großteil der Allokation ist erst beim Prozess der Abtrennung der Kondensatoren im Sankey-Diagramm visualisiert, da auch hier erst eine materielle Trennung des Tantals (Kondensatoren) von den Edelmetallen (restliche Leiterplatte) vorgenommen wird. 


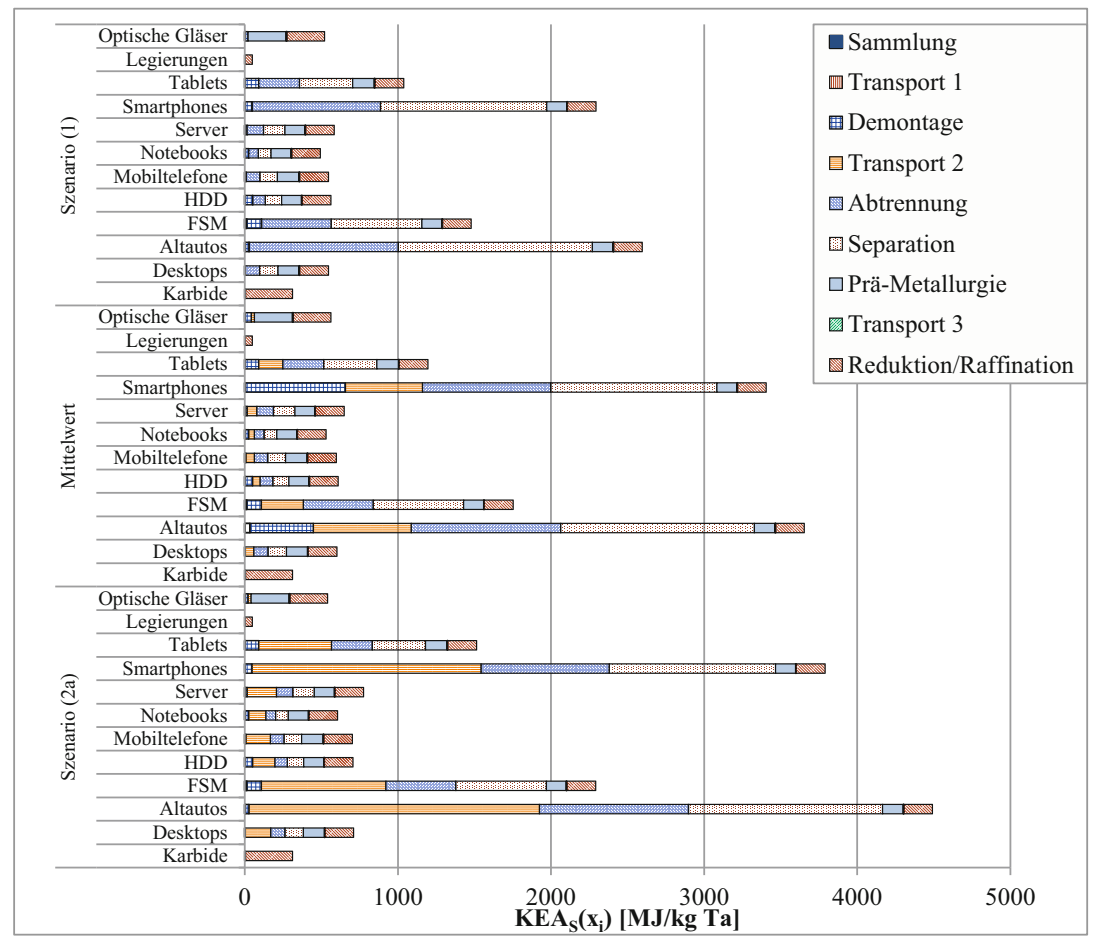

Abbildung 5.12 Allozierte prozessscharfe KEA des Tantalrecyclings aus sekundären Quellen (Mittelwert der Transportszenarien). Hinweis: Das Tantanrecycling von Karbiden und Legierungen findet im Werkstoffverbund statt

Tantalhaltige Kondensatoren sind, wie oben bereits erwähnt, auf Leiterplatten verbaut. Diese kommen wiederum in Produkten zum Einsatz. Die Tantalkonzentration dieser Produkte bzw. der zukünftigen sekundären Quellen errechnet sich demnach durch die Multiplikation der Tantalkonzentration auf den Leiterplatten mit der Konzentration der Leiterplatte am Gesamtprodukt. Sowohl bei den Tantalkonzentrationen der Leiterplatten (Both 2016) als auch bei den Konzentrationen der Leiterplatten an den Produkten, wie z. B. Elektrogeräten (Bookhagen et al. 2018a), waren über die vergangenen Jahre und Jahrzehnte die Trends abnehmender Konzentrationen zu beobachten. Insbesondere die Tantalkonzentrationen an den Leiterplatten haben in den vergangenen Jahrzehnten signifikant abgenommen. Technische Fortschritte ermöglichten die Produktion von immer feinerem Tantalpulver und damit immer höheren Oberflächen des Pulvers bzw. der Pulverkristalle. 
Da sich die Kapazität eines Kondensators proportional zur Oberfläche der Tantalanode bzw. dem daraus bestehenden Tantalpulver verhält, konnte die Kapazität der Kondensatoren seit 1970 um den Faktor 15 gesteigert werden. Gleichzeitig konnte die Größe der Kondensatoren und damit die pro Kondensator benötigte Menge an Tantal um den Faktor 200 verringert werden - die Kondensatoren wurden miniaturisiert. D. h. ein Kondensator aus dem Jahr 2015 ist 200-mal kleiner als einer, der 1970 gebaut wurde, hat jedoch die 15-fache Kapazität. Diese Entwicklung war notwendig, um modernste Produkte wie z. B. Smartphones zu realisieren (Both 2016). Zusätzlich zu dieser Miniaturisierung ist auch die Anzahl der verbauten Kondensatoren auf den Leiterplatten rückläufig, was zu Teilen auf die Kapazitätssteigerung pro Kondensator und auf Substitutionen zurückzuführen ist (Schulenburg 2018; Bookhagen et al. 2018b; Chancerel et al. 2016).

Wie sich die $K E A_{S}\left(x_{i}\right)$ des Tantalrecyclings bei Änderungen in diesen Konzentrationen verhalten, ist in Abbildung 5.13 für die jeweiligen sekundären Quellen untersucht. Auf der Abszisse sind die Tantalkonzentrationen in der gesamten sekundären Quelle angegeben. Dabei ist angenommen, dass die Konzentrationen der Leiterplatte an der sekundären Quelle und die Konzentrationen des Tantals an der Leiterplatte im selben Verhältnis variieren. Die Allokationsfaktoren und Recyclingeffizienzen sind dabei konstant gesetzt. Die Erklärung hierzu ist Abschnitt 5.2.3.2 zu entnehmen. Für die Legierungen und Karbide ist keine Konzentrationsparametrisierung vorgenommen, da diese sekundären Quellen im Materialverbund recycelt werden und die Konzentrationen daher eine nur untergeordnete Rolle spielen.

Die $K E A_{S}\left(x_{i}\right)$ aller sekundären Quellen folgen bei Änderungen der Tantalkonzentrationen einem potenziellen Wachstum. Ein weiteres Absinken der Tantalkonzentrationen würde also unter den getroffenen Annahmen zu einem überproportionalen Wachstum der KEA des Recyclings führen. Die unterschiedlichen Wachstumsverläufe der kondensatorhaltigen sekundären Quellen sind dabei auf die unterschiedlichen Werte der beiden Konzentrationen, die die Gesamtkonzentration bestimmen, zurückzuführen. Fällt der Wachstumsverlauf verhältnismäßig flach aus - Beispiele hierfür sind Server oder Mobiltelefone - so ist die Tantalkonzentration an der Leiterplatte des Produktes aktuell verhältnismäBig hoch. ${ }^{7}$ Denn diese Konzentration hat einen wesentlich größeren Einfluss auf den $K E A_{S}\left(x_{i}\right)$, als die Konzentration der Leiterplatte am Gesamtprodukt. Das ist darauf zurückzuführen, dass die Tantalkonzentration der Leiterplatte für die

\footnotetext{
${ }^{7}$ Wär diese Konzentration bereits sehr niedrig, so würden Änderungen dieser Konzentration einen stärkeren Einfluss auf den KEA haben. Das ist auf die 1/x Beziehung dieser beiden Parameter zurückzuführen.
} 
gleichen Prozesse wie auch die Konzentration der Leiterplatte am Gesamtprodukt ausschlaggebend ist, zudem jedoch auch noch für den Prozess der Abtrennung der Kondensatoren. Dieser Prozess der Kondensatorabtrennung macht aufgrund seiner vollständigen Allokation auf Tantal einen relativ hohen Anteil am $K E A_{S}\left(x_{i}\right)$ aus. Sind die Tantalkonzentrationen in diesem Prozess gering, so werden die $K E A_{S}\left(x_{i}\right)$ dieses Prozesses entsprechend hochskaliert. Konzentrationsänderungen des Tantals in Leiterplatten haben somit einen stärkeren Einfluss auf den $K E A_{S}\left(x_{i}\right)$ des Tantalrecyclings als Konzentrationsänderungen der Leiterplatte am Produkt.

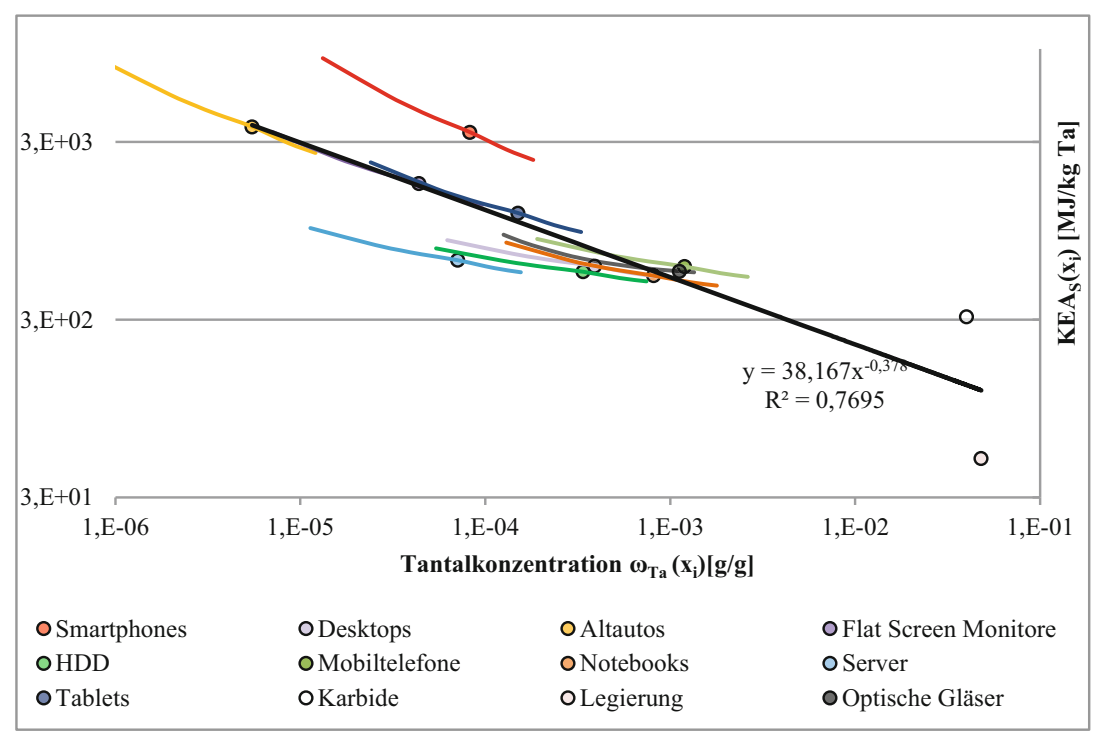

Abbildung 5.13 Auswertung der KEA des Tantalrecyclings nach den Konzentrationen

\subsubsection{Massenbilanzen}

Die ermittelten Massenbilanzen des Tantalrecyclings aus sekundären Quellen beziehen sich auf das Jahr 2013. Dieses Jahr bietet die beste Datengrundlage insbesondere für Kondensatoren die, wie in den vorherigen Abschnitten gezeigt, in 
unterschiedlichsten Produkten verbaut werden und daher eine differenzierte und umfangreiche Datengrundlage benötigen. Diese Datengrundlage ist aus Ueberschaar et al. (2017a) entnommen und durch persönliche Kommunikation mit dem Hauptautor ergänzt (Ueberschaar 2018).

Die Sankey-Darstellung in Abbildung 5.14 visualisiert die Tantalmassenflüsse der sekundären Quellen entlang der Recyclingketten. Aus Gründen der Übersichtlichkeit sind die Kondensatoren als aggregierte sekundäre Quelle abgebildet. Massenaustausch zwischen den sekundären Quellen, wie es u. a. beim Kupferrecycling der Fall ist, existiert hier nicht. Die theoretisch verfügbare Masse an Tantal in den sekundären Quellen betrug für das Jahr 2013 in Deutschland ca. 15,3 t. $75 \%$ davon sind auf die Kondensatoren zurückzuführen, die auch tatsächlich auf den Leiterplatten identifiziert und rückgewonnen werden können. Weitere $7 \%$ sind auf Tantalkondensatoren, die nicht identifizierbar und damit auch nicht rückgewinnbar sind, und auf Tantalbeschichtungen zurückzuführen. Tantalkarbide sind mit $14 \%$ die zweitgrößte sekundäre Quelle. Einer innerdeutschen Sammlung wurden von diesen theoretisch verfügbaren bzw. angefallenen Massen jedoch nur 5,5 t zugeführt. Das ist im Wesentlichen auf die geringen Sammelquoten der Elektroaltgeräte zurüickzuführen. Diese beträgt für Altgeräte aus dem IKTBereich durchschnittlich ca. $54 \%$. Für Smartphones und Mobiltelefone liegt die Sammelquote bei lediglich $18 \%$ (Chancerel 2010). Weitere Informationen zu der Massenbilanz von Tantal sind Abschnitt B.2.2.1 in Appendix II des elektronischen Zusatzmaterials zu entnehmen.

Ebenso wie beim Kupferrecycling sind auch beim Recycling von Tantal die größten Massenverluste ${ }^{8}$ durch die unzureichenden Sammlungen zu verzeichnen. In Abbildung 5.14 sind zudem größere Massenverluste in der mechanischen Aufbereitung des Recyclings aus Kondensatoren zu erkennen. Diese Phase fasst die Demontage der Leiterplatten, die thermische Abtrennung der Kondensatoren und deren anschließende Separation zusammen. Diese Prozessschritte haben zwar jeweils hohe Recyclingeffizienzen $R E\left(x_{i}\right)_{j}$ (siehe Tabelle 5.5), die Recyclingeffizienz der gesamten mechanischen Aufbereitung liegt dennoch bei ca. $64 \%$, da sich die einzelnen Effizienzen über die Recyclingkette hinweg multiplizieren. Die Recyclingeffizienzen $R E\left(x_{i}\right)_{j}$ der metallurgischen Prozesse liegen für alle sekundären Quellen bei weit über $90 \%$ und führen daher zu nur geringen Massenverlusten.

Die Masse an sekundärem Tantal, das durch ein Recycling aus den sekundären Quellen nach aktuellen Sammelquoten und Recyclingeffizienzen in Deutschland erzielt werden könnte, liegt bei 3,8 t/Jahr. Diese Angabe ist rein theoretischer

\footnotetext{
${ }^{8}$ Verluste durch Exporte bzw. ,nicht-Sammeln“ - siehe Abschnitt 5.2.4.
} 


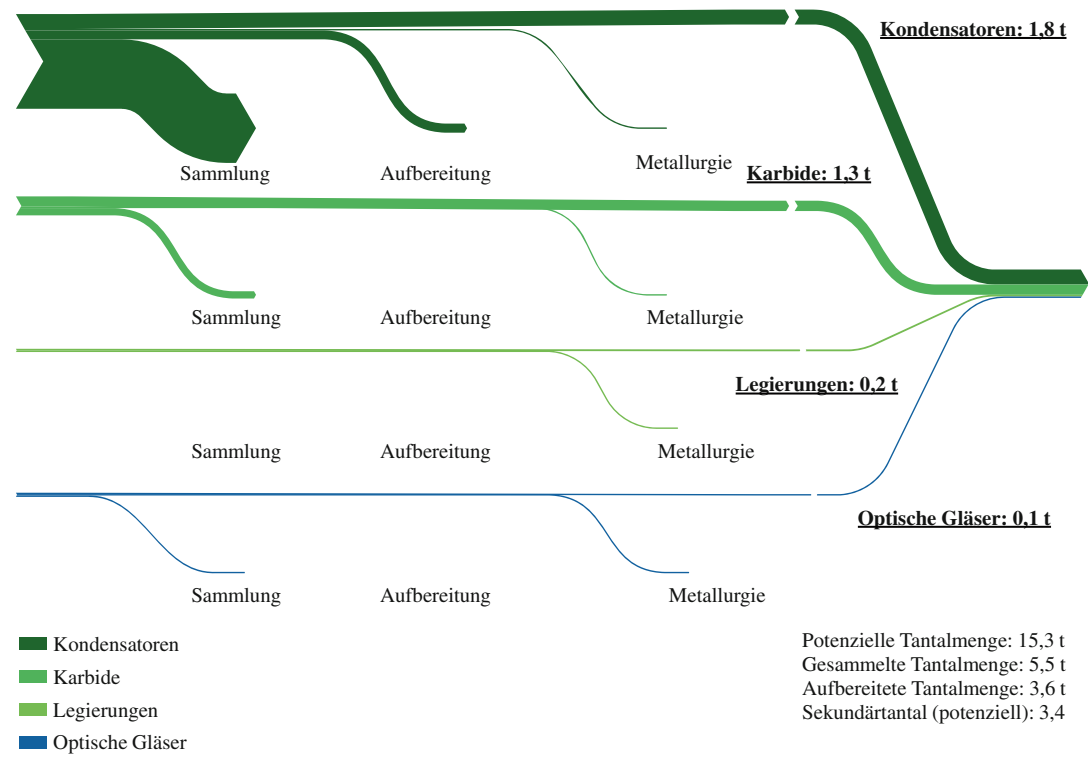

Abbildung 5.14 Massenbilanz des (theoretischen) Tantalrecyclings aus sekundären Quellen für das Jahr 2013 in Deutschland

Natur, da bis dato kein EoL-Recycling von Tantal aus Kondensatoren oder optischen Gläsern stattfindet. Die Massen, die tatsächlich recycelt werden - Tantal aus Legierungen und Karbiden - belaufen sich auf 1,5 t pro Jahr. Die aktuelle EoL-RQ für Tantal beträgt nach diesen Werten ca. $11 \%$.

Die ermittelten Massenbilanzen basieren zu großen Teilen auf statistischen Daten offizieller Einrichtungen und hochqualitativen Fachzeitschriften und sind daher als valide angenommen. Eine umfassende Validitätsprüfung, wie sie bei Kupfer vorgenommen ist, kann jedoch aufgrund des Mangels an Vergleichsdaten nicht vorgenommen werden. Für Tantal existieren in der Literatur bis dato lediglich zwei umfangreichere und differenzierte Massenbilanzen - auf europäischer (Deetman et al. 2017) und globaler Ebene (Nassar 2017). Diese Studien können aufgrund der abweichenden Untersuchungsrahmen jedoch nur annäherungsweise verwendet werden, um die Größenordnungen der ermittelten Massen zu bestätigen. 


\subsubsection{Vergleich der primären und sekundären Gewinnung}

\subsubsection{Aufwände der Primärgewinnung}

Die primäre Gewinnung von Tantal stammt zu ca. $60 \%$ aus artisanalen und kleinbergbaulichen Quellen. Tantal aus industriellem Bergbau beträgt $31 \%$ der jährlichen Fördermenge. Die verbleibenden $9 \%$ sind auf die Gewinnung aus Zinnschlacken zurückzuführen (Damm 2018). Der artisanale Bergbau findet überwiegend in Afrika statt (Damm 2018). Daten zu den Aufwänden, die dabei betrieben werden, sind keine vorhanden. Auch die Datenlage der Aufwände der industriellen Primärgewinnung von Tantal ist eher spärlich und auf zwei Datensätze der führenden Ökobilanzdatenbanken ecoinvent und GaBi beschränkt. Beide Datensätze erfassen die industrielle Tantalgewinnung aus australischen Minen und die anschließende Aufbereitung und Raffination in Europa. Sie kommen jedoch zu unterschiedlichen Ergebnissen in den KEA und $\mathrm{CO}_{2}$ eq-Emissionen. Die Werte sind in Tabelle 5.6 aufgelistet.

Tabelle 5.6 KEA und $\mathrm{CO}_{2}$ eq-Emissionen der primären Tantalgewinnung

\begin{tabular}{l|l|l}
\hline & KEA $[\mathrm{MJ} / \mathrm{kg} \mathrm{Ta}]$ & $\begin{array}{l}\mathrm{CO}_{2} \text { eq-Emissionen } \\
{[\mathrm{kg} / \mathrm{kg} \mathrm{Ta}]}\end{array}$ \\
\hline $\begin{array}{l}\text { ecoinvent } \\
(2011)\end{array}$ & 4.592 & 302 \\
\hline $\begin{array}{l}\text { thinkstep AG } \\
(2018)(\mathrm{GaBi})\end{array}$ & 2.170 & 140 \\
\hline
\end{tabular}

Beide Datensätze basieren nach Angaben der Datenbanken auf Daten aus der industriellen Praxis. Der Datensatz von ecoinvent verweist in einer ausführlichen Dokumentation auf Daten der Firma H.C. Starck GmbH (Classen et al. 2009), dem weltweit größten Tantalproduzenten. Jährlich werden hier ca. 1.000 t Tantal produziert, was über die Hälfte der globalen Jahresproduktion ausmacht. Die Kapazitäten der Firma könnten eine doppelt so hohe Ausbringung ermöglichen (Schulenburg 2018). Eine Besonderheit der metallurgischen Produktionsroute von H.C. Starck ist die Reduktion des Tantaloxids zu hochreinem Tantal mit Magnesiumdampf. Nur so kann eine maximal große Oberfläche des Tantalpulvers erzielt werden, wie sie für den Einsatz in modernsten Kondensatoren benötigt wird (Schulenburg et al. 2017; Schulenburg 2018). Die Dokumentation des GaBiDatensatzes ist deutlich weniger umfangreich und gibt keine Auskunft darüber, aus welchen produzierenden Unternehmen die Daten stammen (thinkstep AG 2018, 2017c). Die Informationen, die über das angenommene Verfahrensschema bereitgestellt sind, lassen jedoch darauf schließen, dass es sich hierbei ebenfalls 
um die Firma H.C. Starck handelt, allerdings um eine leicht abweichende Prozessroute. So wird u. a. keine Magnesiumdampfreduktion eingesetzt, sondern eine Reduktion mit Natrium. Die Qualität des Tantalpulvers, die dabei erzeugt wird, ist geringer als bei der Magnesiumdampfreduktion und ist daher für den Einsatz in Hochleistungskondensatoren nicht ausreichend (H.C. Starck GmbH 2019). Da die meisten Produkte jedoch mittlerweile Hochleistungskondensatoren benötigen (Pitcher 2006), ist der ecoinvent-Datensatz nach dem Anteil der Kondensatoren an den theoretisch verfügbaren Mengen von $75 \%$ (siehe Abschnitt 5.3.4) gewichtet. Der GaBi-Datensatz ist mit den verbleibenden $25 \%$ gewichtet. Der KEA, der für die primäre Tantalgewinnung angesetzt ist, beträgt damit $3.987 \mathrm{MJ} / \mathrm{kg}$ Ta. Die daraus resultierenden $\mathrm{CO}_{2}$ eq-Emissionen belaufen sich auf $262 \mathrm{~kg} / \mathrm{kg}$ Ta.

Für die zukünftige primäre Gewinnung von Tantal ist eine verstärkte Kuppelproduktion mit Lithium prognostiziert (Damm 2018). Durch die damit verbundenen Allokationseffekte würde der KEA ersten Abschätzungen zufolge auf ca. 900 MJ/kg Ta sinken (Rötzer 2019).

\subsubsection{Ermittlung der energetisch optimalen End-of-Life Recyclingquoten}

\subsection{Status Quo und Zukunftsszenario}

Aktuell findet Tantalrecycling nur für die sekundären Quellen Legierungen und Karbide statt. Das gewichtete arithmetische Mittel des KEA $\left(\overline{K E A}_{S}\right)$ beträgt $273 \mathrm{MJ} / \mathrm{kg}$ Ta, bei einer EoL-RQ von ca. $11 \%$. Die damit verbundenen $\mathrm{CO}_{2}$ eqEmissionen belaufen sich auf $16 \mathrm{~kg} / \mathrm{kg}$ Ta. Würden alle gesammelten sekundären Quellen zu den angegebenen Recyclingeffizienzen recycelt werden, so läge der gewichtete Mittelwert für das Recycling von $1 \mathrm{~kg}$ Ta bei ca. $563 \mathrm{MJ}$ und $34 \mathrm{~kg} \mathrm{CO}$ eq-Emissionen. Die fiktive EoL-RQ beträgt in diesem Fall $25 \%$. Die Datengrundlage dieser Berechnungen ist in Tabelle 5.7 enthalten.

Das durchschnittliche EoL-Tantalrecycling, wie es derzeit praktiziert wird, benötig nach den Modellergebnissen pro kg Tantal einen $93 \%$ geringeren KEA und verursacht $94 \%$ geringere $\mathrm{CO}_{2}$ eq-Emissionen als die primäre Gewinnung. In Summe werden aktuell somit ca. 5,5 TJ und 361 t $\mathrm{CO}_{2}$ eq-Emissionen durch das EoL-Tantalrecycling pro Jahr eingespart. Die Optimierung der EoL-RQ nach den aktuellen Sammelquoten und Recyclingeffizienzen der Primärgewinnung ist in Abbildung 5.15a veranschaulicht. Da die $K E A_{S}\left(x_{i}\right)$ aller sekundären Quellen unter dem durchschnittlichen KEA der Primärgewinnung von $3.987 \mathrm{MJ} / \mathrm{kg} \mathrm{Ta}$ liegen, ist das Tantalrecycling aus allen diesen sekundären Quellen energetisch vorteilhaft. Demnach liegt das energetische Optimum der EoL-RQ nach den aktuellen Sammelquoten $S Q\left(x_{i}\right)$ und den technisch möglichen Recyclingeffizienzen $R E\left(x_{i}\right)_{j}$ bei $25 \%$. 


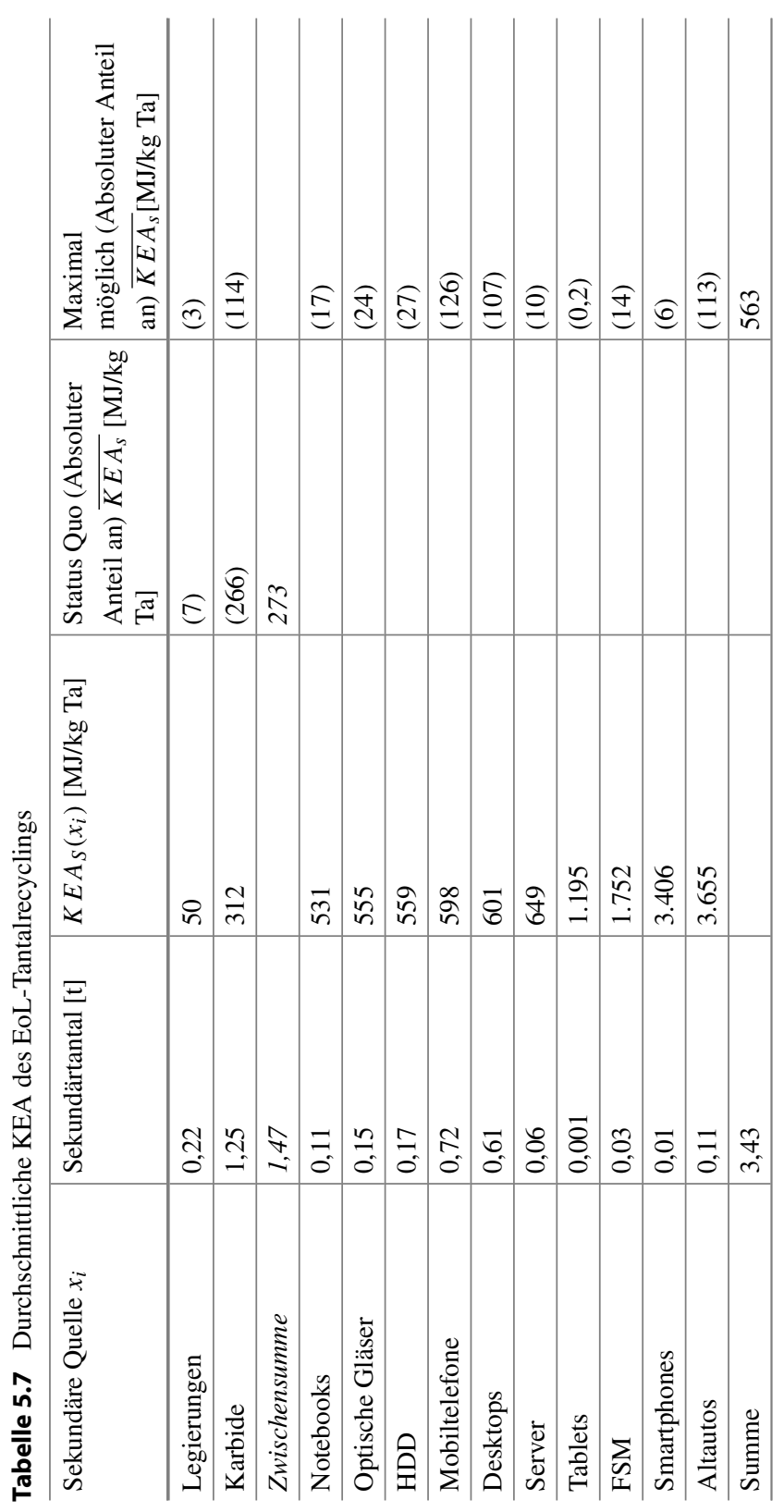



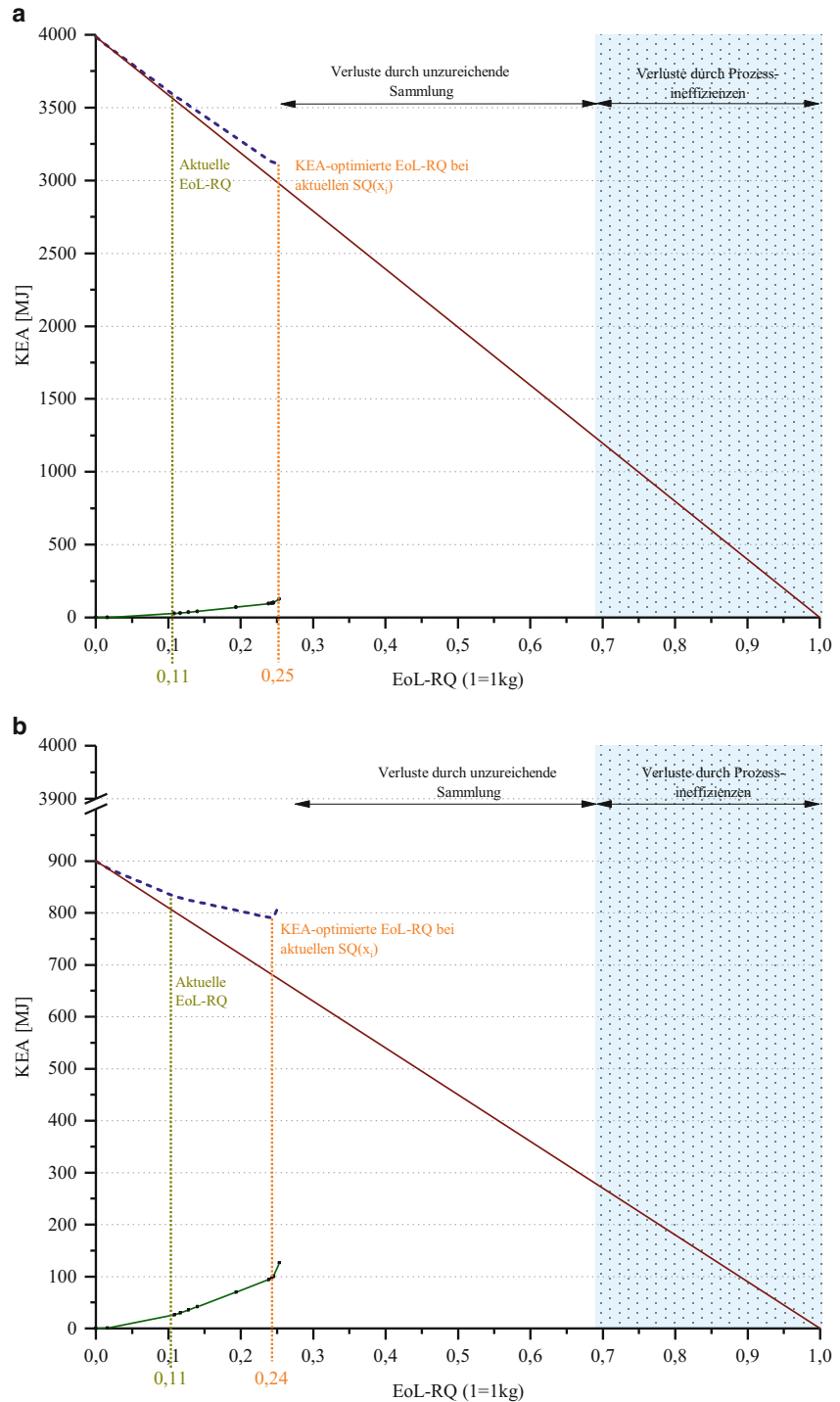

- - - - Total in MJ/kg — KEA des Primäranteils —— KEA des Recyclinganteils bei aktuellen SQ $\left(\mathrm{x}_{\mathrm{i}}\right)$

Abbildung 5.15 KEA-optimierte EoL-RQ des Tantalrecyclings a der aktuellen Verhältnisse und $\mathbf{b}$ der abgeschätzten zukünftigen Verhältnisse der primären Tantalgewinnung 
Die derzeitigen Recyclingaktivitäten bis zu dieser KEA-optimierten EoL-RQ zu erweitern, würde die Gesamtersparnisse des Tantalrecyclings in Deutschland auf 11,7 TJ und $780 \mathrm{t} \mathrm{CO}_{2}$ eq pro Jahr erhöhen. Die $K E A_{S}\left(x_{i}\right)$ der beiden sekundären Quellen mit dem energieintensivsten Recycling (Smartphones und Altautos) liegen allerdings nur geringfügig unterhalb des KEA der primären Gewinnung (siehe Tabelle 5.7). Marginale Änderungen in den Prozessdaten bzw. Tantalkonzentrationen - siehe hierzu die Auswertungen in Abschnitt 5.3.3.2 - können dazu führen, dass diese $K E A_{S}\left(x_{i}\right)$ die Schwelle des KEA der Primärgewinnung überschreiten und deren Recycling so zu negativen Trade-offs führen würde.

Für die zukünftigen Entwicklungen ist ein KEA von $900 \mathrm{MJ}$ pro kg primär gewonnenes Tantal angenommen. Durch diese Absenkung der KEA der primären Gewinnung liegen die vier aufwandsintensivsten sekundären Quellen (Altautos, Smartphones, FSM und Tablets) nicht mehr innerhalb der KEA-optimierten EoL-RQ. Die tatsächlichen Auswirkungen auf die KEA-optimierte EoL-RQ sind jedoch aufgrund der geringen Massen dieser sekundären Quellen nur geringfügig. Sie verschiebt sich von $25 \%$ auf $24 \%$. Die Ergebnisse sind in Abbildung 5.15b veranschaulicht. Da diese Produktgruppen derzeitig hohen Wachstumsraten unterliegen, sind hier jedoch in Zukunft Änderungen in den Massenbilanzen der sekundären Quellen und damit auch der KEA-optimierten EoL-RQ zu erwarten.

Die vorgenommenen Auswertungen berücksichtigen nur Änderungen des KEA in der primären Gewinnung. Mögliche Änderungen der KEA des Recyclings können - wie in Abschnitt 5.3.3.2 beschrieben - z. B. durch Änderungen der Tantalkonzentrationen der sekundären Quellen entstehen. Nach den Ergebnissen des Abschnitt 5.3.3.2 können bei diesem Zukunftsszenario die Tantalkonzentrationen der sekundären Quellen, die innerhalb der KEA-optimierten EoL-RQ liegen, um bis zu $40 \%$ sinken, ohne dass sie aus der KEA-optimierten EoL-RQ der Abbildung 5.15b fallen. Die $K E A_{S}\left(x_{i}\right)$ des Tantalrecyclings aus den sekundären Quellen, die nach dem angenommenen Zukunftsszenario höher ausfallen als die primäre Gewinnung, müssten um 25-75\% reduziert werden, um energieeffizienter zu sein als die Primärgewinnung. Diese Effizienzgewinne müssten durch technische Effizienzsteigerungen realisiert werden. Dass signifikante Erhöhungen der Tantalkonzentrationen stattfinden, ist aufgrund des beobachteten Trends der sinkenden Konzentrationen als unwahrscheinlich einzustufen.

\subsection{Szenarien-Analyse}

Im vorangestellten Abschnitt ist gezeigt, dass eine Erweiterung der EoL-RQ auf bis zu $25 \%$ zu energetischen Ersparnissen führen kann. Weitere Erhöhungen der EoL-RQ sind zudem durch Verbesserungen der Sammelquoten $S Q\left(x_{i}\right)$ möglich. 
Geht man von der in Abschnitt 4.4.2 begründeten Annahme aus, dass eine Erhöhung der Sammelquote $S Q\left(x_{i}\right)$ nicht in einer Erhöhung der $K E A_{S}\left(x_{i}\right)$ resultiert, würde sich die KEA-optimierte EoL-RQ auf $69 \%$ erhöhen. Die Ersparnisse an KEA und $\mathrm{CO}_{2}$ eq-Emissionen könnten damit um jeweils den Faktor 5,6 gesteigert werden. Die grafische Darstellung der Optimierung dieses Szenarios ist in Abbildung 5.16a veranschaulicht.

Um zu untersuchen, wie sich Erhöhung der Sammelquoten $S Q\left(x_{i}\right)$ auf die KEA-optimierte EoL-RQ auswirken, ist ein weiteres Szenario gerechnet, bei dem für die zusätzlich gesammelten Massen die Auslastung der Transportkapazitäten auf ein Minimum von $5 \%$ reduziert und die Allokationsmethode auf eine ökonomische Allokation abgeändert ist. Die Begründung dieses Szenarios kann Abschnitt 5.2.5.2.2 entnommen werden. Die Auswertung ist in Abbildung 5.16b veranschaulicht. Die Massen der sekundären Quellen, die durch die erhöhten Sammelquoten $S Q\left(x_{i}\right)$ unter energetischem Mehraufwand gesammelt werden, resultieren in höheren $K E A_{S}\left(x_{i}\right)$. Diese sind in Abbildung 5.16b als gesonderte diskrete Datenpunkte (graue Datenpunkte) aufgeführt. Trotz wesentlicher Erhöhungen der Sammelaufwände, die in Erhöhungen der $K E A_{S}\left(x_{i}\right)$ um bis zu über $300 \%$ resultieren, verbleibt die KEA-optimierte EoL-RQ bei $69 \%$. Es kann somit angenommen werden, dass Verbesserungen der Sammelquoten $S Q\left(x_{i}\right)$ auch bei signifikant steigenden Sammelaufwänden zu weiteren energetischen Ersparnissen führen.

Die verfügbaren Recyclingverfahren der größten sekundären Quelle - den EoL-Kondensatoren - befinden sich aktuell noch im Pilotmaßstab bzw. kleinindustriellen Maßstab. Es kann deshalb davon ausgegangen werden, dass hier noch weitere Verbesserungen der Recyclingeffizienzen $R E\left(x_{i}\right)$ realistisch sind, insbesondere durch die Umsetzung in den großindustriellen Maßstab. Wie sich Änderungen der Recyclingeffizienzen $R E\left(x_{i}\right)_{j}$ auf die $K E A_{S}\left(x_{i}\right)$ und damit auf die KEA-optimierte EoL-RQ auswirken, kann, wie in Abschnitt 4.4.2 erläutert, in der vorliegenden Arbeit nicht quantitativ ermittelt werden.

\subsubsection{Herausforderungen des Tantalrecyclings}

Die Auswertungen des vorherigen Abschnitt 5.3.5.2 zeigen, dass das Tantalrecycling aus Kondensatoren, das bis dato noch nicht stattfindet, zu signifikanten Einsparungen an Energie und THGE führen kann. Neben der in den vergangenen Jahren und Jahrzehnten beobachteten Miniaturisierung, die bereits in 

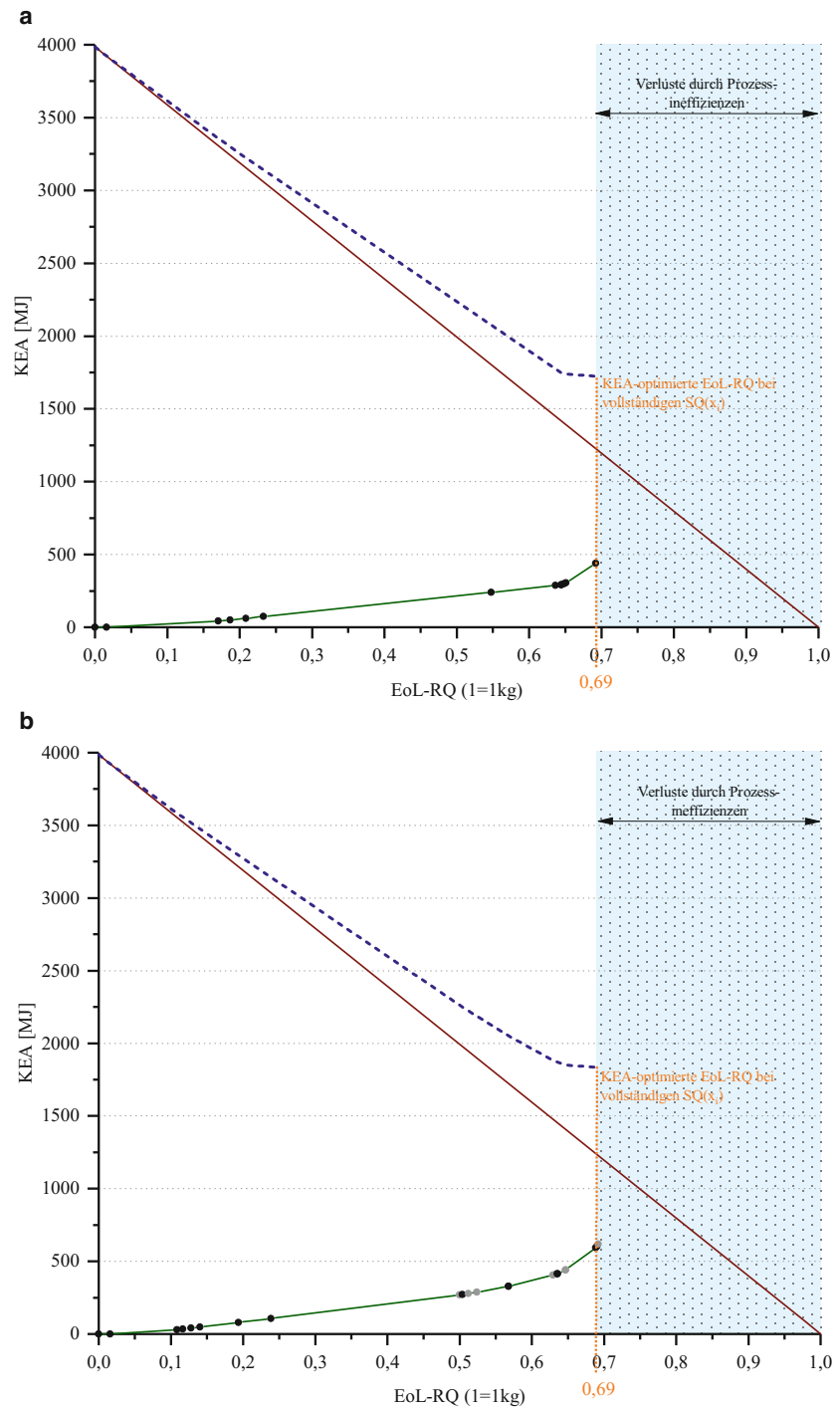

$-\cdots$ Total in MJ/kg $\longrightarrow$ KEA des Primäranteils $\multimap$ KEA des Recyclinganteils bei vollständigen SQ( $\left.\mathrm{x}_{\mathrm{i}}\right)$

Abbildung 5.16 KEA-optimierte EoL-RQ des Tantalrecyclings bei vollständigen Sammelquoten und a konstanten Sammelaufwänden pro kg Ta bzw. b variablen Sammelaufwänden pro kg Ta (5 \% Auslastung der Transportkapazitäten, ökonomische Allokation) 
Abschnitt 5.3.3.2 untersucht ist, existieren beim Tantalrecycling aus Kondensatoren noch zwei weitere Herausforderungen - die Wirtschaftlichkeit und mögliche Zielkonflikte durch Silberverluste - die in diesem Abschnitt untersucht sind.

\subsubsection{Verfügbare Mengen und Wirtschaftlichkeit}

Die im Jahr 2013 theoretisch verfügbaren Massen an Tantal in EoLKondensatoren aus in Deutschland angefallenen Elektroaltgeräten betrugen $21 \mathrm{t}$. Davon wurden nur knapp $11 \mathrm{t}$ einer Sammlung zugeführt. Mit dem aktuellen Marktpreis für Tantal von ca. $215 € / \mathrm{kg}$ (USGS 2019) und den Verlusten durch die Recyclingeffizienzen über die Recyclingkette von $43 \%$ bedeutet das einen möglichen Umsatz von 1,3 Mio. $€$. Eine dezentrale Demontage der Kondensatoren bei den deutschlandweit ca. 400 Erstbehandlungsanlagen ist für die einzelnen Anlagen aus wirtschaftlicher Sicht uninteressant. Nimmt man eine Gleichverteilung der Kondensatoren auf die Erstbehandlungsanlagen an, würden pro Jahr auf eine Anlage nur wenige Kilogramm Tantal bzw. Materialwerte im niedrigen vierstelligen Bereich kommen. Die verfügbaren Mengen an Tantalkondensatoren sind somit zu gering, um eine dezentrale Demontage wirtschaftlich zu verwirklichen. Dieses Problem könnte über eine zentralisierte Demontage der Kondensatoren und eine dezentrale Demontage der Leiterplatten gelöst werden. Letzteres ist $\mathrm{zu}$ weiten Teilen ohnehin gängige Praxis, da Leiterplatten hohe Anteile an Kupfer und Edelmetallen enthalten und aufgrund dieser hohen Werthaltigkeit demontiert und gezielt recycelt werden (Fröhlich 2018c). Mit einer zentralisierten Lösung und einem oder wenigen spezialisierten Unternehmen auf nationaler oder sogar internationaler Ebene, die die Separation der Kondensatoren vornehmen, kann das Recycling von Tantal aus Kondensatoren wirtschaftlich werden. Zudem werden die hohen prognostizierten Wachstumsraten des Tantalbedarfs für Kondensatoren von einem Faktor 8,4 bis 2035 weitere Skaleneffekte ermöglichen (Marscheider-Weidemann et al. 2016).

\subsubsection{Zielkonflikt durch Silberverluste}

Neben Tantal und den weniger werthaltigen Materialien wie Epoxidharz oder den Eisen-Nickel-Terminals sind in Tantalkondensatoren geringe Mengen an Silber (ca. $3 \%$ ) enthalten. Das oben angenommene Recyclingverfahren für Tantal aus Kondensatoren ermöglicht keine Rückgewinnung dieses Silberanteils. Wird kein gezieltes Tantalrecycling aus den Kondensatoren vorgenommen, verbleiben die Kondensatoren auf der Leiterplatte und werden mit weiteren kupferhaltigen Fraktionen in die Kupfermetallurgie eingebracht. In diesem Fall kann das Silber am Ende der metallurgischen Prozesskette rückgewonnen werden (siehe Abschnitt 5.2.3 und Abschnitt B.1.1.2 in Appendix II des elektronischen Zusatzmaterials). 
Es existiert somit ein Zielkonflikt zwischen dem Recycling von Tantal und dem Recycling von Silber aus Kondensatoren, die u. a. von Ueberschaar et al. (2017a) als wesentliches Hemmnis des Tantalrecyclings eingestuft sind.

Die Silberverluste können der Argumentation dieser Arbeit zufolge als Aufwände des Tantalrecyclings verstanden werden bzw. vice versa. Um diese Aufwände zu quantifizieren, sind die KEA, die durch die Substitution von primärem Silber eingesparten werden, abzüglich der KEA, die mit dem Silberrecycling über die Kupfermetallurgie verbunden sind, errechnet. ${ }^{9}$ Durch die Subtraktion dieser Aufwände der Silberverluste von den theoretischen Ersparnissen des Tantalrecyclings ${ }^{10}$ kann überprüft werden, welche dieser beiden Optionen energetisch vorteilhafter ist. Die Auswertung in Abbildung 5.17 zeigt, dass die Silberverluste bei sieben der neun kondensatorhaltigen sekundären Quellen kaum Auswirkungen auf die Gesamtersparnis des Tantalrecyclings haben. Lediglich die beiden sekundären Quellen Altautos und Smartphones werden in ihren KEA-Ersparnissen durch die Silberverluste um einen Großteil reduziert, was auf die relativ hohen Aufwände des Tantalrecyclings aus diesen sekundären Quellen zurückzuführen ist. Sie liegen dennoch im positiven Bereich der Nettoersparnisse. Das Tantalrecycling ist also für alle sekundären Quellen dem Silberrecycling aus energetischer Sicht vorzuziehen.

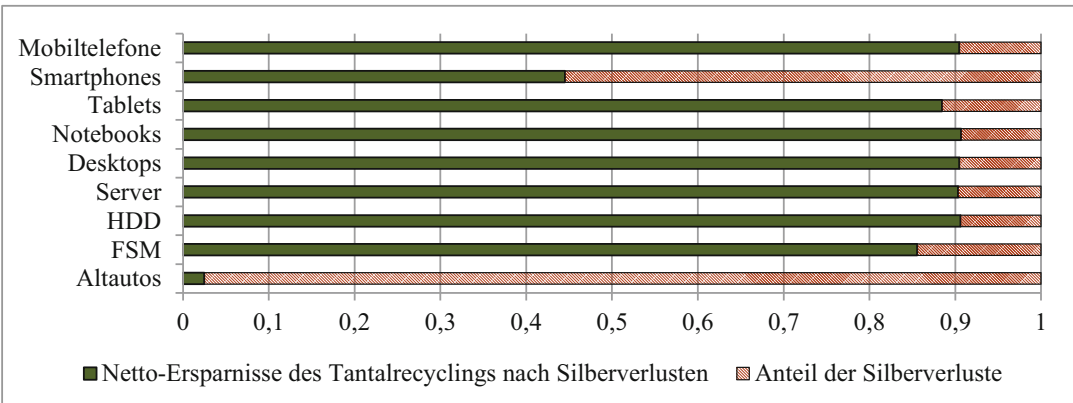

Abbildung 5.17 Nettoersparnisse des Tantalrecyclings aus Kondensatoren nach Abzug der Silberverluste in relativen Werten

\footnotetext{
${ }^{9}$ Die Werte der primären Silbergewinnung und des Aufwandes des Silberrecyclings über die Kupfermetallurgie sind der ecoinvent Datenbank entnommen.

${ }^{10}$ KEA des substituierten Primärmaterials abzüglich des KEA des Recyclings. Es sind die KEA der aktuellen primären Gewinnung von $3.987 \mathrm{MJ} / \mathrm{kg}$ angesetzt.
} 


\subsubsection{Fazit zum Tantalrecycling}

Die Tantalkonzentrationen in den untersuchten sekundären Quellen weisen eine enorme Spannweite von knapp $5 \%$ bis 5 ppm auf. Die sekundären Quellen mit den niedrigsten Konzentrationen sind Elektroaltgeräte und elektronische Komponenten in Altautos, deren Tantalgehalt auf die Kondensatoren auf Leiterplatten zurückzuführen ist. Diese sekundären Quellen machen den größten Anteil an verfügbarem Tantal für ein EoL-Recycling aus. Aktuell findet jedoch kein EoL-Recycling von Tantal aus Kondensatoren statt. Durch die geringsten Konzentrationen und die komplexe Verbauung von Kondensatoren in den Produkten, benötigt das Tantalrecycling entsprechend viele Prozessschritte und resultiert in hohen $K E A_{S}\left(x_{i}\right)$ von ca. 560 bis zu $3.700 \mathrm{MJ} / \mathrm{kg}$ Ta.

Aktuell finden lediglich die sekundären Quellen mit hohen Tantalkonzentrationen (Legierungen und Karbide) den Weg in ein Recycling. Die EoL-RQ beträgt in Deutschland derzeit ca. $11 \%$, die globale EoL-RQ ist auf $<1 \%$ geschätzt (Graedel et al. 2011). Der Vergleich der modellbasierten $K E A_{S}\left(x_{i}\right)$ mit den KEA der primären Tantalgewinnung zeigt jedoch, dass ein Recycling aus allen untersuchten sekundären Quellen aus energetischer und klimabilanzieller Sicht sinnvoll wäre. Bei den aktuellen Sammelquoten und Recyclingeffizienzen liegt die KEA-optimierte EoL-RQ bei ca. $25 \%$. Geht man von einer vollständigen Sammlung aus, könnte die EoL-RQ auf maximal $69 \%$ erhöht werden. Die $K E A_{S}\left(x_{i}\right)$ des Recyclings aus den aufwandsintensivsten Quellen liegt allerdings nur knapp unterhalb des KEA der primären Tantalgewinnung. Geringfügige Änderungen in den sekundär- oder primärseitigen Aufwänden können also zu einer Verschiebung der KEA-optimierten EoL-RQ führen. In welchem Umfang eine solche Verschiebung stattfinden könnte, zeigt das erstellte Zukunftsszenario. Hier ist von einer rapiden Abnahme der KEA der Primärgewinnung durch eine Verschiebung auf eine vermehrte Kuppelproduktion ausgegangen. Die Auswirkungen auf die KEA-optimierte EoL-RQ sind jedoch nur gering, da die aufwandsintensivsten sekundären Quellen derzeit noch in verhältnismäßig geringen Massen vorkommen.

Die Untersuchungen des Beispielmetalls Tantal zeigen, dass das Recycling von gering konzentrierten Technologiemetallen durchaus mit deren Primärgewinnung konkurrieren und zu Einsparungen an Energie und THGE führen kann. Ein wesentlicher Aspekt ist hierbei, dass die tantalhaltigen Kondensatoren auf der meist gut zu demontierenden Leiterplatte verbaut sind. Durch deren Demontage kann je nach sekundärer Quelle mit relativ geringem Aufwand eine Konzentrationserhöhung um den Faktor 4 bis 60 realisiert werden. Damit wird der 
Konzentrationseffekt wesentlich abgeschwächt, was dazu führt, dass das Recycling von Tantal, auch aus sekundären Quellen mit geringeren Konzentrationen als die der primären Quellen, einen geringen KEA benötigt.

Um die identifizierten Einsparpotenziale einer Erhöhung der EoL-RQ von Tantal trotz der geringen verfügbaren Massen auch in der Praxis zu realisieren, sind eine intelligente Transportlogistik, eine zentralisierte Abtrennung der Kondensatoren von den Leiterplatten und eine intensive Vernetzung aller beteiligten Akteure notwendig. Nur so können Skaleneffekte realisiert werden, die für eine wirtschaftliche Aufbereitung unumgänglich sind.

\section{$5.4 \quad$ Kobalt}

\subsubsection{Die Bedeutung von Kobalt}

Die erste Verwendung von Kobalt geht auf das Jahr 2.000 v. Chr. zurück. Ägyptische Handwerker haben Kobaltsalze als Färbemittel verwendet, allerdings vermutlich ohne etwas über die Existenz von Kobalt zu wissen. Der erste Nachweis, dass die blaufärbende Eigenschaft auf das nun entdeckte Element Kobalt zurückzuführen ist, wurde im Jahr 1735 durch den Schweden Georg Brandt vorgenommen (Wang 2006). Auch heute findet Kobalt noch Anwendung als Färbemittel, allerdings nur noch zu geringen Anteilen. Die Hauptanwendungen sind mittlerweile Karbide, Superlegierungen und vor allem Lithium-Ionen-Batterien (LIB) für Elektrogeräte und Elektroautos (Al Barazi et al. 2018). Insbesondere Superlegierungen und LIB sind als Zukunftstechnologien identifiziert, deren Bedarfswachstum bis zum Jahr 2035 auf den Faktor 3 für Legierungen und den Faktor 92 für LIB prognostiziert ist (Marscheider-Weidemann et al. 2016).

Im Jahr 2017 wurden weltweit etwa 118.500 t Kobalt aus primären Quellen gefördert. Das primärseitige Angebot wird aktuell zu $84 \%$ aus dem industriellen Bergbau, als Kuppelprodukt des Kupfer- oder Nickelbergbaus, gedeckt. Die verbleibenden $16 \%$ stammen aus dem artisanalen Bergbau, der schwerpunktmäßig in der demokratischen Republik Kongo (DR Kongo) stattfindet. Auch der industrielle Bergbau wird zu großen Teilen in der DR Kongo vorgenommen, die in Summe $64 \%$ des gesamten primärseitigen Kobaltangebots bereitstellt (Al Barazi et al. 2018). Insbesondere aufgrund dieses hohen Anteils der DR Kongo - einem relativ instabilen Land - und der hohen Bedeutung des Kobalts für die Wirtschaft, ist Kobalt für viele Wirtschaftsräume als kritisches Metall eingestuft (EC 2017b; Erdmann und Behrendt 2011; Graedel et al. 2015). Die durchschnittliche Konzentration in den derzeitig betriebenen Minen liegt bei 0,2 \% (BGS 2009a). Mit einer 
durchschnittlichen Konzentration in der Erdkruste von 25 ppm ist Kobalt halb so häufig vertreten wie Kupfer (Al Barazi et al. 2018).

Vor allem die Herstellung und das Recycling von LIB, die bereits heute $44 \%$ der globalen Nachfrage nach Kobalt ausmachen (Al Barazi et al. 2018), stehen derzeit häufig in den Schlagzeilen und werden dort meist aufgrund von ihnen zugeschriebenen Umweltbelastungen an den Pranger gestellt (Süddeutsche Zeitung 2018; Frankfurter Allgemeine 2019; Fokus 2019). Das Recycling von Kobalt spielt damit auch bei der ökobilanziellen Betrachtung der Verkehrswende eine entscheidende Rolle. Denn die Umweltwirkungen eines Elektroautos sind maßgeblich von den LIB beeinflusst (Nordelöf et al. 2014).

\subsubsection{Sekundäre Kobaltquellen}

Die Anwendungsgebiete von Kobalt sind vielfältig. Neben den LIB in Elektrogeräten und Elektroautos kommt Kobalt auch in Nickel-Metallhydrid-Batterien (NiMHB) zum Einsatz. Die Verwendung als Kathodenmaterial in Batterien ist mit $46 \%$ das größte Anwendungsgebiet (Stand 2017). Das zweitgrößte Anwendungsgebiet sind mit $19 \%$ Superlegierungen ${ }^{11}$ (nachfolgend als Legierungen bezeichnet). Diese finden hautsächlich in Flugzeugturbinen ihren Einsatz (Harper et al. 2012). Die Verwendung von Kobalt als Karbid in Schneid- und Bohrwerkzeugen macht weitere $9 \%$ aus. Mit diesen Anwendungen sind $74 \%$ der globalen Nachfrage abgedeckt. Abbildung 5.18 zeigt die prozentuale Aufteilung aller Anwendungsgebiete von Kobalt für das Jahr 2017.

Die übrigen der in Abbildung 5.18 aufgeführten Anwendungen für Kobalt sind größtenteils dissipativer Natur und somit von einem potenziellen Recycling ausgenommen. Hierunter fallen z. B. die Kategorien weitere Anwendungen und weitere chemische Anwendungen. Kobalt wird hier u. a. als Salz in Tierfutter oder auch als Chlorid in pharmazeutischen Produkten eingesetzt. Als Karbonat findet es Anwendung als Färbemittel in der Glas-, Email- und Keramikindustrie. In metallischer Form wird es in der Oberflächenveredelung eingesetzt (Al Barazi et al. 2018). Der Einsatz von Kobalt in Magneten muss bis dato ebenfalls als dissipative Anwendung eingeordnet werden. Bisherige Verfahren zu Magnetrecycling befassen sich ausschließlich mit der Rückgewinnung von Seltenen Erden (Hagelüken 2018b). Eine Anwendung, die ebenfalls unter die Kategorie weitere chemische Anwendungen fällt, sind industrielle Katalysatoren - hierzu existieren bereits großindustrielle Recyclingverfahren (Hassan 2003), es sind

\footnotetext{
${ }^{11}$ Hier sind auch Schnellarbeitersstähl mit ca. $2 \%$ der globalen Nachfrage integriert.
} 


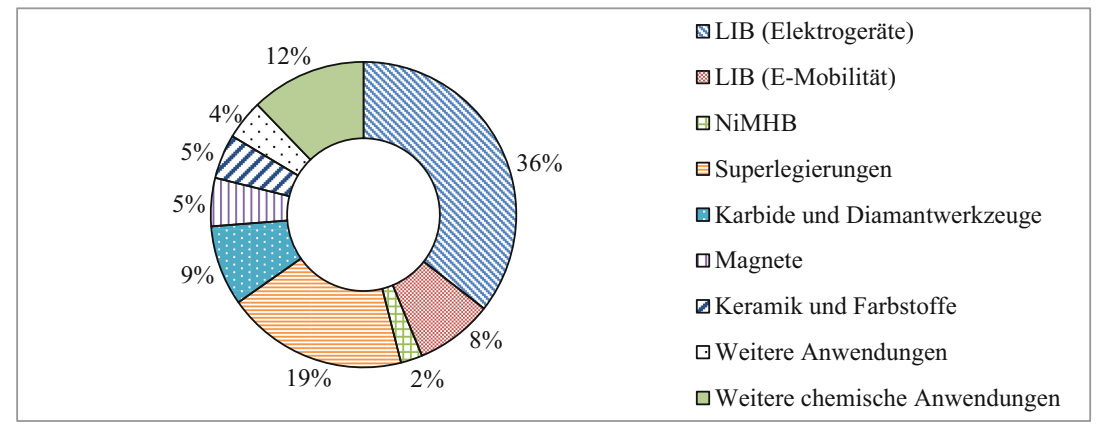

Abbildung 5.18 Hauptanwendungsgebiete Kobalt (2017) (Al Barazi et al. 2018)

jedoch weder massenbilanzielle noch prozessspezifische Daten deren Recycling verfügbar. Katalysatoren können daher ebenfalls nicht als sekundäre Quelle in die nachfolgenden Untersuchungen integriert werden. Durch ihren relativ geringen Massenanteil am Gesamtkobaltverbrauch und ihre langen Lebenszeiten sind die Einflüsse der Katalysatoren auf die Sekundärkobaltmassenbilanz ohnehin marginal. Zudem ist die Kobaltkonzentration in Katalysatoren mit ca. $2 \%$ verhältnismäßig hoch (Gaballah et al. 1994; Jong et al. 1989), es liegt hier deshalb kein nennenswertes Konzentrationsproblem vor.

Die untersuchten sekundären Quellen der nachfolgenden Modelle und Auswertungen sind LIB, NiMHB, Legierungen und Karbide. Da LIB und NiMHB zu unterschiedlichen Größen bzw. Anteilen in unterschiedlichsten Produkten verbaut werden - was sich wiederum entscheidend auf die Kobaltkonzentrationen der jeweiligen sekundären Quelle auswirkt - ist eine differenzierte Betrachtung der Produkte notwendig. Im Bereich der Elektromobilität kann eine Unterscheidung zwischen Elektroautos und Hybridautos vorgenommen werden. Eine Betrachtung der Elektrogeräte auf Produktebene, wie im Falle von Tantalkondensatoren in Abschnitt 5.3 vorgenommen, ist aufgrund der unzureichenden Datenlage nicht möglich. Es müsste für jedes Produkt, das eine kobalthaltige Batterie enthält, bekannt sein, welchen Massenanteil die Batterie am Gesamtprodukt ausmacht. Diese Daten sind nur für wenige Produkte vorhanden - u. a. für Mobiltelefone (bzw. Smartphones) und Notebooks (Hagelüken 2018a). Diese beiden Produkte sind die größten Anwendungsgebiete für LIB in Elektrogeräten (Leuthner 2013). Ein ähnlicher Schwerpunkt der Anwendung war in der Vergangenheit auch bei NiMHB gegeben (Müller und Friedrich 2006), also bei den Produkten, die heute noch als EoL-Produkte anfallen. Das ist auch durch Angaben 
der Urban Mining Plattform (2018) sowie der dazugehörigen Veröffentlichung bestätigt (Huisman et al. 2017). Die beiden Produkte sind in Punkto Batterieanteil und Produktgesamtgewicht repräsentativ für zahlreiche weitere Elektrogeräte (Kameras, Smartphones, Tablets etc.). Die Vielzahl an Elektrogeräten ist daher auf zwei Kategorien aggregiert - kleine Elektronikprodukte mit Batterieanwendung $^{12}$, repräsentiert durch Mobiltelefone und große Elektronikprodukte mit Batterieanwendung ${ }^{13}$, repräsentiert durch Notebooks.

Die kobalthaltigen LIB in Elektrogeräten lassen sich in die zwei Batterietechnologien mit unterschiedlichen Zusammensetzungen Lithium-Kobaltdioxid $\left(\mathrm{LiCoO}_{2}\right)$ und Lithium-Nickel-Mangan-Kobalt-Oxid (LiNMC) unterteilen (Urban Mining Plattform 2018). Alle in den nachfolgenden Modellen und Auswertungen betrachteten sekundären Quellen sind in Tabelle 5.8 mit ihren jeweiligen Konzentrationen aufgeführt.

Tabelle 5.8 Sekundäre Kobaltquellen und deren Kobaltkonzentrationen (Datengrundlage in Kapitel B.3 des Appendix II des elektronischen Zusatzmaterials)

\begin{tabular}{l|l}
\hline sekundäre Quelle & $\omega_{C o}\left(x_{i}\right)[\mathrm{g} / \mathrm{g}]$ \\
\hline Hybridaltautos LIB & 0,0006 \\
\hline Elektroaltautos LIB & 0,0042 \\
\hline NiMH-Batterie Elektroaltgeräte groß & 0,0119 \\
\hline NiMH-Batterie Elektroaltgeräte klein & 0,0146 \\
\hline LiNMC-Batterie Elektroaltgeräte groß & 0,0145 \\
\hline LiNMC-Batterie Elektroaltgeräte klein & 0,0178 \\
\hline LiCoO 2 -Batterie Elektroaltgeräte groß & 0,0329 \\
\hline LiCoO 2 -Batterie Elektroaltgeräte klein & 0,0403 \\
\hline Karbide & 0,1100 \\
\hline Legierung & 0,3250 \\
\hline
\end{tabular}

\footnotetext{
${ }^{12}$ Beinhaltet Mobiltelefone, Smartphones, Tablets und Kameras.

${ }^{13}$ Beinhaltet Notebooks, Werkzeuge und E-Bikes. Für E-Bikes ist lediglich der Batterieanteil und nicht das Produktgesamtgewicht entscheidend, da die Demontage durch die einfache Entnahme der Batterie erfolgt.
} 


\subsubsection{Recyclingmodell}

\subsubsection{Recyclingverfahren und Prozessdaten}

Die sekundären Kobaltquellen lassen sich in drei Gruppen zusammenfassen, die jeweils spezifische Recyclingverfahren benötigen: Batterien, Karbide und Legierungen. Die Recyclingverfahren von Karbiden und Legierungen sind bereits am Beispiel Tantal in Abschnitt 5.3.3.1 erläutert und an dieser Stelle nicht erneut erwähnt. Für kobalthaltige Batterien existieren zahlreiche großindustrielle Recyclingverfahren, die in verschiedenen Unternehmen zum Einsatz kommen (Rothermel et al. 2018; Treffer 2011; Zeng et al. 2014). Tabelle 5.9 gibt einen Überblick der Unternehmen und deren Recyclingverfahren, die derzeit die größten Kapazitäten aufweisen. Detaillierte Beschreibungen der verfügbaren Recyclingverfahren können Pinegar und Smith (2019) entnommen werden.

Für die nachfolgenden Analysen ist das Umicore-Verfahren ausgewählt. Diese Auswahl hat mehrere Gründe. Zum einen existiert für dieses Verfahren eine sehr gute und valide Datenlage, was eine detaillierte Modellierung ermöglicht. Zum anderen verfügt die Umicore NV/SA über die größten Kapazitäten und hat somit einen maßgeblichen Anteil am Gesamtsystem des Batterierecyclings. Zudem ist dieser Recyclingprozess für LIB und NiMHB jeglicher Größe geeignet (Treffer 2013; Hagelüken und Treffer 2016) und kann damit gleichermaßen für das Recycling von Batterien aus Elektroaltgeräten und aus Altautos herangezogen werden.

Tabelle 5.9 Übersicht der wichtigsten Unternehmen und Recyclingverfahren für kobalthaltige Batterien (Treffer 2011)

\begin{tabular}{l|l|l|l|l}
\hline Unternehmen & Accurec GmbH & Batrec AG & Umicore NV/SA & Toxco Inc. \\
\hline Standort & $\begin{array}{l}\text { Mühlheim } \\
\text { (Deutschland) }\end{array}$ & $\begin{array}{l}\text { Wimmis } \\
\text { (Schweiz) }\end{array}$ & Hoboken (Belgien) & Trail (Kanada) \\
\hline $\begin{array}{l}\text { Kapazität } \\
{[\mathrm{t} / \mathrm{a}]}\end{array}$ & 2.500 & 5.000 & 7.000 & 3.500 \\
\hline Zielmetalle & $\mathrm{Fe}, \mathrm{Cu}, \mathrm{Mn}, \mathrm{Co}$ & $\begin{array}{l}\mathrm{Fe}, \mathrm{Cu}, \mathrm{Mn}, \mathrm{Zn}, \\
\mathrm{Co}\end{array}$ & $\mathrm{Fe}, \mathrm{Cu}, \mathrm{Mn}, \mathrm{Ni}, \mathrm{Co}$ & $\mathrm{Co}$ \\
\hline Verfahren & Vakuumthermisch & Materialtrennung & $\begin{array}{l}\text { Pyro- und } \\
\text { Hydrometallurgisch }\end{array}$ & Pyrometallurgisch \\
\hline
\end{tabular}

Der erste Prozessschritt eines jeden Recyclingverfahrens von Batterien ist die Demontage bzw. Entnahme aus dem EoL-Produkt. Für Batterien aus kleinen Elektroaltgeräten ist eine nachgelagerte Sortierung angenommen (Fisher et al. 2006). 
Nach Angaben von Hagelüken (2018a) müssen die aus den Altautos demontierten Batterien einer weiteren Demontage unterzogen werden. Dabei werden die Batterien bis auf die Zellebene zerlegt. Das Batteriegehäuse und weitere Bestandteile werden ebenfalls weiterführenden Aufbereitungen zugeführt (Buchert et al. 2011). Alle Batterien aus Elektroaltgeräten können ohne weitere Behandlung direkt in die metallurgische Verfahrensroute eingebracht werden. Die metallurgische Route besteht aus einem pyrometallurgischen und einem darauffolgenden hydrometallurgischen Verfahren. Die Verfahrensroute beginnt mit dem Einschmelzen der Batterien und dem Reduzieren aller Oxide in die metallische Form. Grafit und Kohlenstoff, die Bestandteile der Batteriezelle sind, dienen dabei als Reduktionsmittel. Plastik, das ebenfalls Bestandteil der Batteriezelle ist, unterstützt zudem die Befeuerung des Prozesses (Dunn et al. 2012). Der beim Einschmelzen entstehende Flugstaub, der auch reich an Wertstoffen ist, wird in den Prozess rückgeführt. Aluminium und Lithium werden in diesem Prozess als Schlacke ausgetragen und dienen als Zuschlagstoff in der Zementindustrie. Alternativ existieren hier hydrometallurgische Aufbereitungsverfahren, um Lithium aus der Schlacke rückzugewinnen (Treffer 2013). Diese Verfahren benötigen nach den verfügbaren Daten jedoch noch sehr viel mehr Energie, als durch die Substitution von Primärmaterial eingespart werden kann (Buchert et al. 2011), und gelten zudem als nicht wirtschaftlich (Rombach und Friedrich 2019). Nach aktuellen Aussagen ist das Lithiumrecycling mittlerweile jedoch gewinnbringend in das Umicore-Verfahren integriert (Hagelüken 2019). Aktuelle Prozessdaten hierzu sind jedoch nicht verfügbar. Ob dieser Prozess der Lithiumrückgewinnung tatsächlich ökologisch und ökonomisch vorteilhaft ist, kann aufgrund der fehlenden Daten nicht ermittelt werden.

Alle weiteren Metalle, die nicht über die Schlacke ausgetragen werden, reichern sich in einer Legierung an (Treffer 2013). Diese wird ausgehärtet und in einem mechanischen Zwischenschritt auf $100 \mu \mathrm{m}$ granuliert. Das erzeugte Granulat wird in zwei aufeinanderfolgende Leachingprozesse eingebracht, die zuerst Kupfer, dann Eisen, Mangan und, sofern es in der Legierung enthalten ist, auch Zink als Salze ausbringen. Die Inputmenge der Schwefelsäure ist dabei von der Konzentration der auszubringenden Metalle abhängig. In einem anschließenden Solvent Extraction Prozess wird Nickel als Sulfat $\left(\mathrm{NiSO}_{4}\right)$ chemisch ausgelöst. In diesem Prozess entsteht zudem $\mathrm{Co}_{2} \mathrm{Cl}$ als Produkt, was in einem weiterführenden Oxidationsschritt $\mathrm{zu} \mathrm{Co}_{3} \mathrm{O}_{4}$ oxidiert wird. An dieser Stelle des Verfahrens wird in der Praxis das gewonnene Kobaltoxid mit $\mathrm{LiCO}_{3} \mathrm{zu} \mathrm{LiCoO} 2$ reagiert, was wiederum in neuen Batterien zum Einsatz kommt (Treffer 2013; Dunn et al. 2012). Eine weitere Alternative ist das Reduzieren des Kobaltoxides zu reinem Kobalt 
- was auch dem Untersuchungsrahmen dieser Studie entspricht. Dieser Reduktionsschritt zu reinem Kobalt ist in den Modellen angenommen, aufgrund der fehlenden praktischen Anwendung jedoch als optional zu betrachten. Die Reaktion $\mathrm{zu} \mathrm{LiCoO}_{2}$ ist nicht modelliert, da sie nicht in den Untersuchungsrahmen der Studie fällt. Abbildung 5.19 fasst die Verfahrensroute des Recyclings für kobalthaltige Batterien nach dem Umicore-Verfahren zusammen.

Die prozessscharfen Daten des Kobaltrecyclingmodells sind für alle untersuchten sekundären Quellen in Tabelle 5.10 enthalten. Die Dokumentation der Datenerhebung sowie eine ausführliche Beschreibung der Recyclingprozesse sind in Kapitel B.3 des Appendix II des elektronischen Zusatzmaterials enthalten.

Die Sammeltransporte der Batterien entsprechen denen der Elektroaltgeräte bzw. der Altautos. Von den Sammelstellen erfolgt ein weiterer Transport (Transport 1) zu den Aufbereitungsanlagen. Die Transportdistanzen der Sammlung und des Transportes 1 sind bereits in Abschnitt 5.2.3.1 ermittelt. Bei den Aufbereitungsanlagen findet die Demontage der Batterien aus den Elektroaltgeräten bzw. den Altautos statt. Für die demontierten Batterien der Elektroaltgeräte ist der direkte Transport (Transport 2) nach Hoboken in Belgien zu den pyro- und hydrometallurgischen Anlagen der Umicore NV/SA angenommen. Die Batterien aus Altautos werden vor der metallurgischen Aufbereitung zu der weiterführenden Demontagefabrik der Umicore NV/SA in Hanau transportiert (Transport 2). Das dort demontierte kobalthaltige Zellmaterial wird anschließend ebenfalls nach

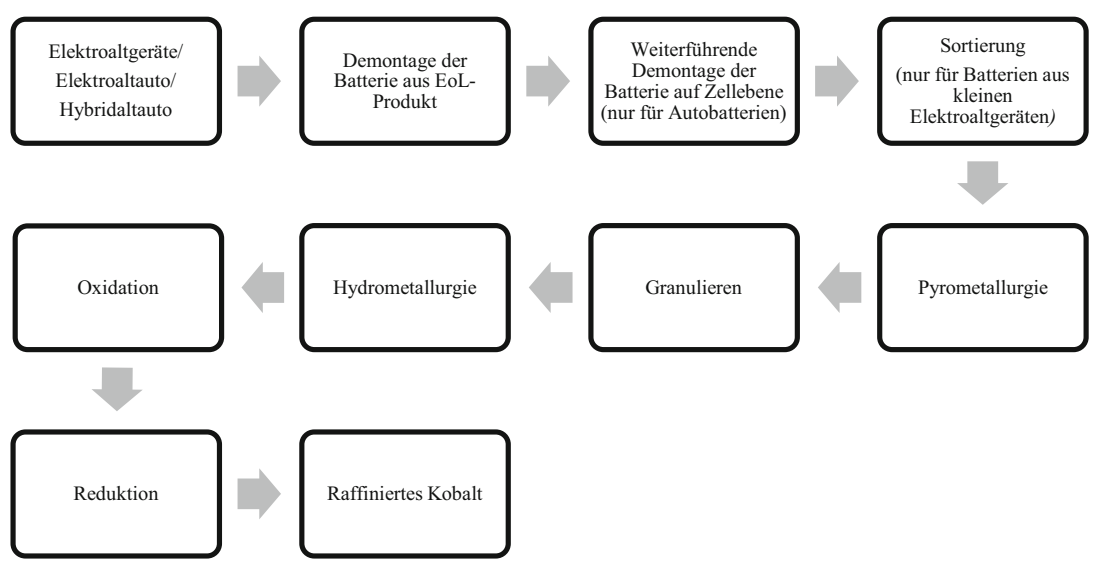

Abbildung 5.19 Verfahren des Recyclings von kobalthaltigen Batterien nach dem UmicoreVerfahren 
政

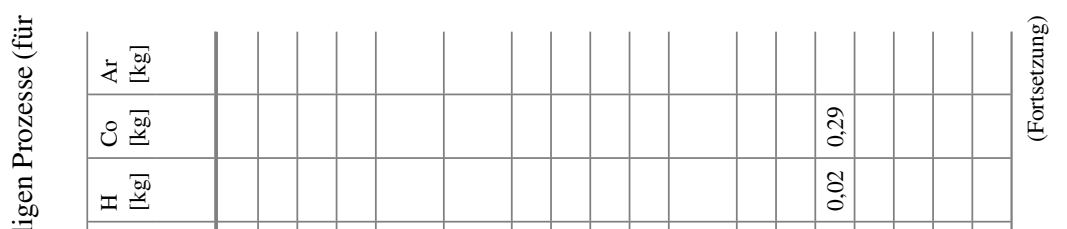

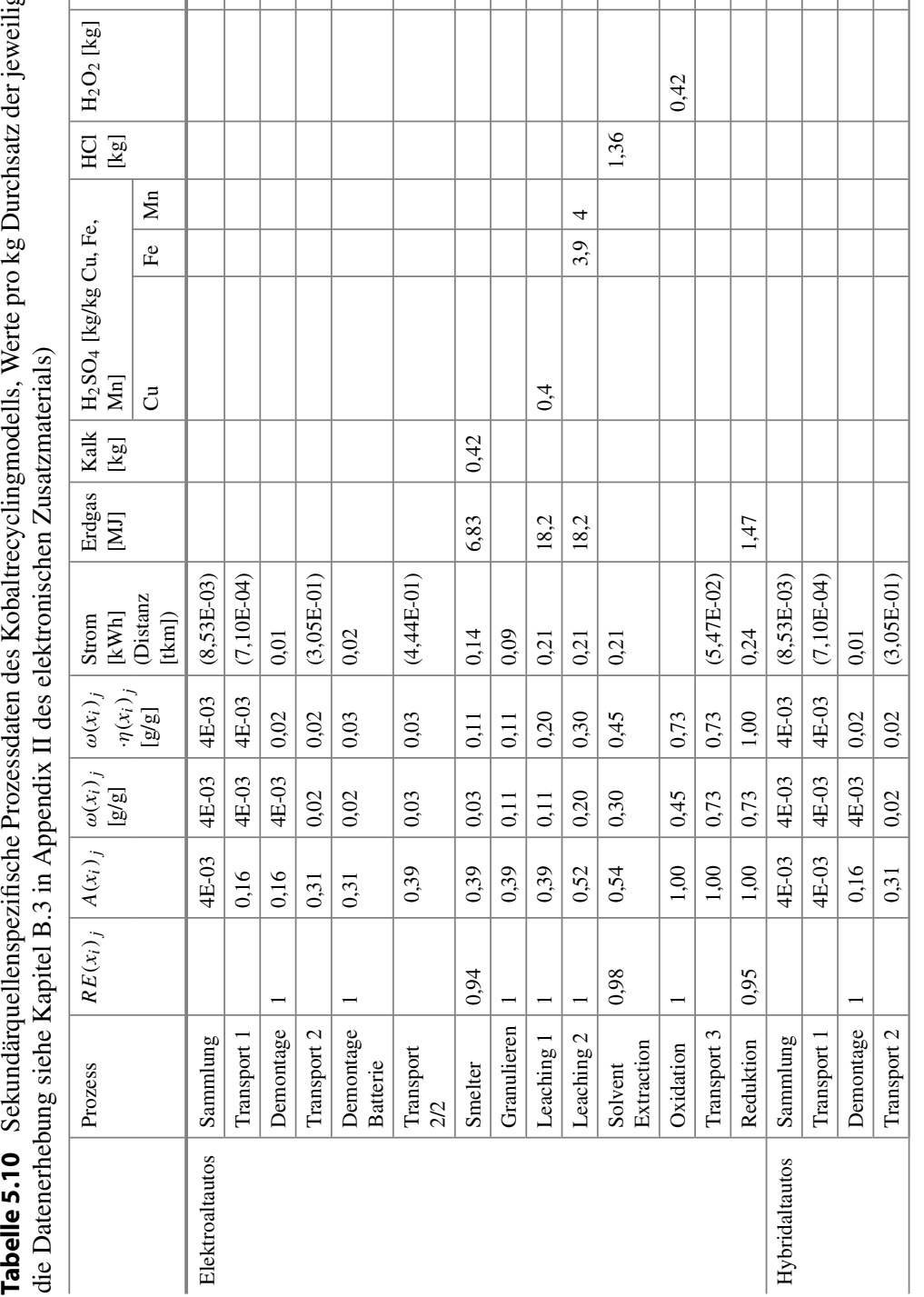




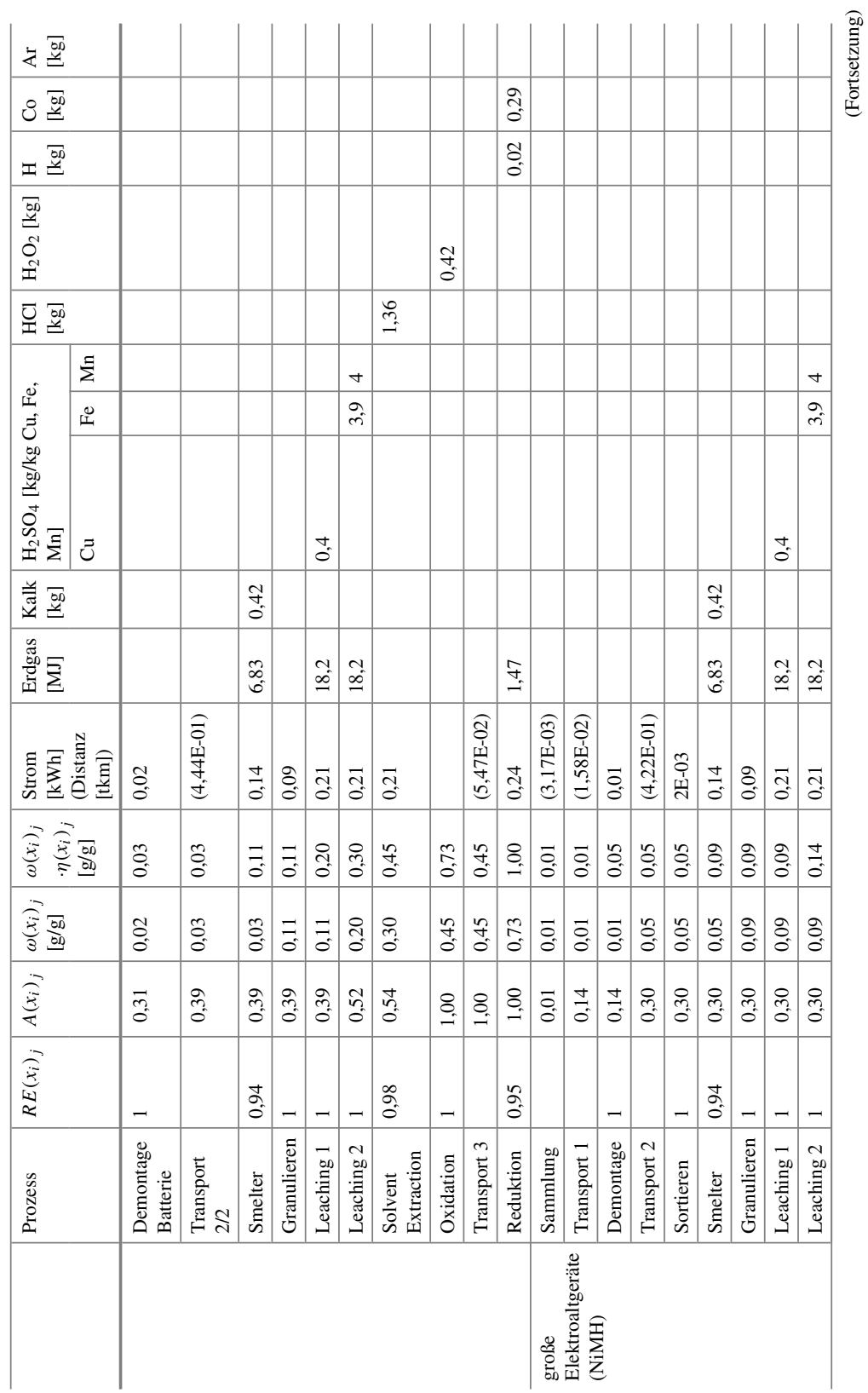




\begin{tabular}{|c|c|c|c|c|c|c|c|c|c|c|c|c|c|c|c|c|c|c|c|c|}
\hline \multicolumn{21}{|l|}{ 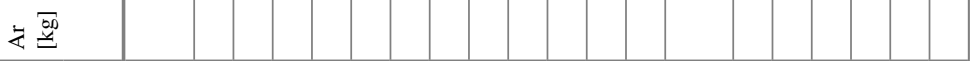 } \\
\hline ن & & & & సे & & & & & & & & & & & & & ते & & & \\
\hline$I \stackrel{50}{\check{D}}$ & & & & $\begin{array}{l}\Omega \\
\delta \\
\delta\end{array}$ & & & & & & & & & & & & & $\stackrel{\sigma}{0}$ & & & \\
\hline 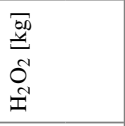 & & $\stackrel{1}{\stackrel{0}{0}}$ & & & & & & & & & & & & & $\stackrel{1}{\stackrel{0}{0}}$ & & & & & \\
\hline 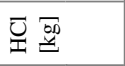 & $\stackrel{\sim}{\sim}$ & & & & & & & & & & & & & 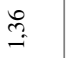 & & & & & & \\
\hline \multirow{3}{*}{ 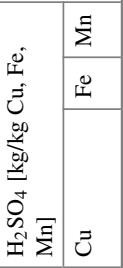 } & & & & & & & & & & & & & $\nabla$ & & & & & & & \\
\hline & & & & & & & & & & & & & ले & & & & & & & \\
\hline & & & & & & & & & & & & ث̈ & & & & & & & & \\
\hline 㮍 & & & & & & & & & & કั. & & & & & & & & & & \\
\hline 总 & & & & $\stackrel{f}{=}$ & & & & & & $\begin{array}{l}\infty \\
\infty \\
0 \\
0\end{array}$ & & 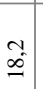 & $\begin{array}{l}\stackrel{1}{\infty} \\
\infty \\
\infty\end{array}$ & & & & $\stackrel{f}{*}$ & & & \\
\hline 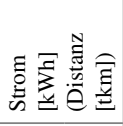 & $\overrightarrow{\tilde{o}}$ & & 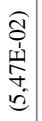 & 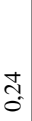 & 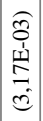 & 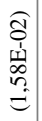 & $\frac{9}{0}$ & 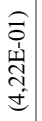 & 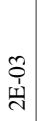 & $\frac{ \pm}{0}$ & $\begin{array}{l}8 \\
0 \\
0\end{array}$ & $\overrightarrow{\tilde{o}}$ & $\overrightarrow{\tilde{o}}$ & $\overrightarrow{\tilde{\sigma}}$ & & & $\underset{⿱}{\Delta}$ & 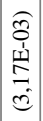 & 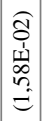 & : \\
\hline 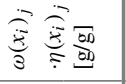 & in & $\frac{m}{0}$ & $\stackrel{n}{0}$ & 8 & $\begin{array}{l}0 \\
0 \\
0\end{array}$ & $\begin{array}{l}0 \\
0 \\
0\end{array}$ & $\begin{array}{l}n \\
0 \\
0\end{array}$ & $\begin{array}{l}n \\
0 \\
0\end{array}$ & $\begin{array}{l}n \\
0 \\
0\end{array}$ & $\begin{array}{l}8 \\
\dot{0}\end{array}$ & $\begin{array}{l}0 \\
\dot{0}\end{array}$ & $\begin{array}{l}0 \\
\dot{0}\end{array}$ & $\begin{array}{l} \pm \\
0\end{array}$ & 年 & $\stackrel{m}{\hat{0}}$ & $\stackrel{m}{\tilde{0}}$ & $\underset{-}{8}$ & $\begin{array}{l}0 \\
0 \\
0\end{array}$ & $\begin{array}{l}0 \\
0 \\
0\end{array}$ & $\stackrel{\circ}{\circ}$ \\
\hline (E) & $\frac{\Delta}{0}$ & $\begin{array}{l}\text { fo } \\
0\end{array}$ & $\stackrel{n}{0}$ & $\stackrel{2}{0}$ & $\begin{array}{l}\bar{\sigma} \\
0 \\
0\end{array}$ & $\tilde{O}$ & $\overrightarrow{0}$ & $\begin{array}{l}8 \\
0 \\
0\end{array}$ & $\ddot{0}$ & $\begin{array}{l}28 \\
0 \\
0\end{array}$ & $\begin{array}{l}8 \\
0 \\
0\end{array}$ & $\begin{array}{l}8 \\
0 \\
0\end{array}$ & $\begin{array}{l}8 \\
0 \\
0\end{array}$ & $\frac{ \pm}{0}$ & 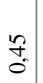 & $\stackrel{\overbrace{}}{0}$ & $\hat{0}$ & $\begin{array}{l}\bar{\sigma} \\
0 \\
0\end{array}$ & $\begin{array}{l}0 \\
0 \\
0\end{array}$ & $\overrightarrow{0}$ \\
\hline 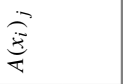 & $\bar{m}$ & $\underset{-}{8}$ & $\underset{-}{8}$ & 8 & $\begin{array}{l}\sigma \\
0 \\
0\end{array}$ & $\frac{n}{0}$ & $\frac{n}{0}$ & $\begin{array}{c}0 \\
\tilde{0} \\
0\end{array}$ & $\begin{array}{c}0 \\
\text { r. } \\
0 \\
0\end{array}$ & 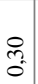 & $\begin{array}{c}\text { m. } \\
0 \\
0\end{array}$ & $\begin{array}{l}0 \\
\vdots \\
0 \\
0\end{array}$ & $\begin{array}{l}0 \\
\text { m. } \\
0\end{array}$ & $\bar{m}$ & $\stackrel{-}{-}$ & $\underset{-}{8}$ & 8 & $\begin{array}{l}0 \\
0 \\
0\end{array}$ & $\frac{7}{0}$ & $\stackrel{ \pm}{0}$ \\
\hline$\underset{\substack{\tilde{E} \\
\multirow{2}{\approx}{}}}{\stackrel{2}{2}}$ & $\stackrel{\infty}{\circ}$ & - & & $\stackrel{n}{2}$ & & & - & & - & gू & - & - & - & $\stackrel{\infty}{\circ}$ & - & & $\approx$ & & & - \\
\hline \multirow[t]{2}{*}{ 岕 } & 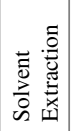 & 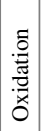 & 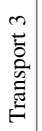 & 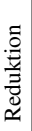 & 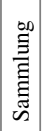 & 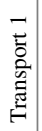 & 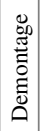 & 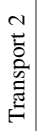 & 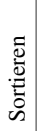 & 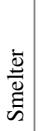 & 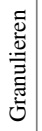 & 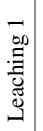 & 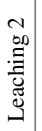 & 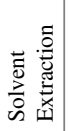 & 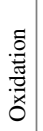 & 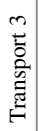 & 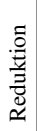 & 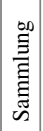 & 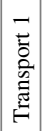 & 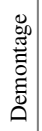 \\
\hline & & & & & & 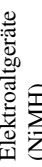 & & & & & & & & & & & & & 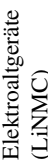 & \\
\hline
\end{tabular}




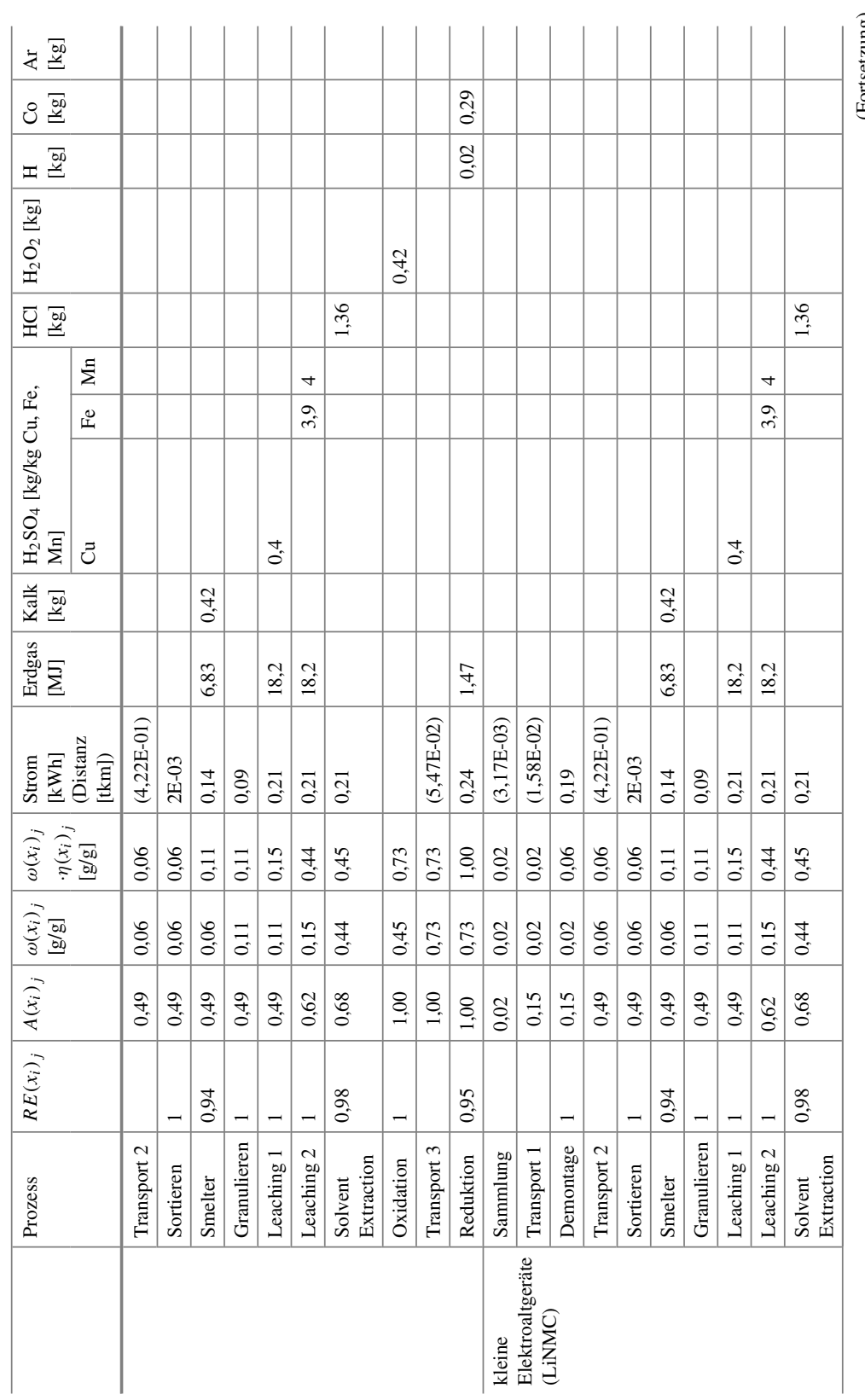




\begin{tabular}{|c|c|c|c|c|c|c|c|c|c|c|c|c|c|c|c|c|c|c|c|c|}
\hline \multicolumn{21}{|l|}{ を安害 } \\
\hline ن & & & సे. & & & & & & & & & & & & & సे & & & & \\
\hline$工 \stackrel{\overline{50}}{=}$ & & & $\begin{array}{l}\mathrm{O} \\
\mathrm{O} \\
0\end{array}$ & & & & & & & & & & & & & ô. & & & & \\
\hline 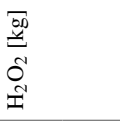 & $\stackrel{y}{0}$ & & & & & & & & & & & & & $\stackrel{\text { I }}{0}$ & & & & & & \\
\hline $\begin{array}{l}\bar{U} \\
\bar{y}\end{array}$ & & & & & & & & & & & & & $\stackrel{\overbrace{}}{-}$ & & & & & & & \\
\hline \multirow{3}{*}{ 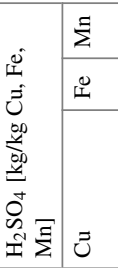 } & & & & & & & & & & & & $\nabla$ & & & & & & & & \\
\hline & & & & & & & & & & & & ले & & & & & & & & \\
\hline & & & & & & & & & & & $\dot{0}_{0}^{+}$ & & & & & & & & & \\
\hline 羔哭 & & & & & & & & & $\stackrel{f}{\mathcal{O}}$ & & & & & & & & & & & \\
\hline 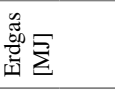 & & & f & & & & & & $\begin{array}{l}0 \\
0 \\
0 \\
0\end{array}$ & & $\underset{\infty}{\infty}$ & \begin{tabular}{l}
\multicolumn{1}{c}{} \\
$\infty$ \\
$\infty$
\end{tabular} & & & & $\stackrel{f}{=}$ & & & & \\
\hline 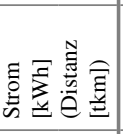 & & 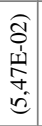 & 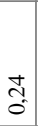 & 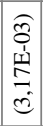 & 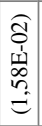 & o. & 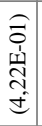 & 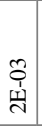 & $\begin{array}{l} \pm \\
0\end{array}$ & $\begin{array}{l}8 \\
0 \\
0\end{array}$ & $\overrightarrow{\tilde{J}}$ & $\overrightarrow{\widetilde{c}}$ & $\overrightarrow{\widetilde{\sigma}}$ & & 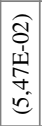 & ذِ & 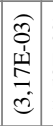 & 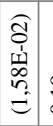 & $\frac{9}{0}$ & 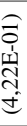 \\
\hline 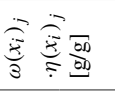 & $\stackrel{n}{0}$ & $\stackrel{n}{0}$ & $\underset{-}{8}$ & $\begin{array}{l}0 \\
0 \\
0 \\
0\end{array}$ & $\begin{array}{l}\tilde{O} \\
\tilde{0} \\
0\end{array}$ & $\frac{n}{0}$ & $\frac{n}{0}$ & $\frac{n}{0}$ & $\begin{array}{l}q \\
+ \\
0\end{array}$ & $\begin{array}{l}q \\
+ \\
0\end{array}$ & $\begin{array}{l}\dot{1} \\
\text { o. } \\
0\end{array}$ & $\underset{-}{8}$ & $\stackrel{\text { rof }}{0}$ & $\stackrel{m}{\tilde{0}}$ & $\frac{m}{0}$ & 8 & $\begin{array}{l}0 \\
0 \\
0 \\
0\end{array}$ & $\begin{array}{l}\dot{0} \\
\dot{0} \\
0\end{array}$ & $\frac{n}{0}$ & $\frac{n}{0}$ \\
\hline $\begin{array}{l}\bar{E} \\
\frac{3}{3}\end{array}$ & $\stackrel{\text { ñ }}{9}$ & $\stackrel{2}{0}$ & $\stackrel{2}{\stackrel{0}{0}}$ & $\begin{array}{l}8 \\
0 \\
0\end{array}$ & $\begin{array}{l}8 \\
0 \\
0\end{array}$ & 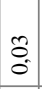 & $\frac{n}{0}$ & $\frac{2}{0}$ & $\frac{n}{0}$ & $\begin{array}{l}q \\
0 \\
0\end{array}$ & $\begin{array}{l}q \\
q \\
0\end{array}$ & $\begin{array}{l}\text { tut } \\
0 \\
0\end{array}$ & $\stackrel{8}{-}$ & $\stackrel{n}{\circ}$ & $\stackrel{m}{0}$ & $\stackrel{2}{0}$ & $\begin{array}{l}0 \\
0 \\
0 \\
0\end{array}$ & $\begin{array}{l}\dot{0} \\
0 \\
0\end{array}$ & $\begin{array}{l}t \\
0 \\
0\end{array}$ & $\frac{n}{0}$ \\
\hline $\bar{E}$ & $\underset{-}{8}$ & $\underset{-}{8}$ & $\underset{-}{8}$ & $\begin{array}{l}0 \\
0 \\
0\end{array}$ & $\begin{array}{l}\infty \\
\stackrel{1}{0} \\
0\end{array}$ & \begin{tabular}{l}
$\infty$ \\
\multirow{1}{1}{} \\
0
\end{tabular} & $\begin{array}{c}\infty \\
\infty \\
0 \\
0\end{array}$ & $\begin{array}{l}\stackrel{0}{\infty} \\
\dot{0}\end{array}$ & $\begin{array}{l}2 \\
\dot{0} \\
0\end{array}$ & $\begin{array}{c}\dot{0} \\
\dot{0} \\
\dot{0}\end{array}$ & $\begin{array}{l}2 \\
\dot{0} \\
0\end{array}$ & ळ. & $\stackrel{8}{-}$ & $\underset{-}{-}$ & $\stackrel{8}{-}$ & $\stackrel{8}{-}$ & $\mid \begin{array}{l}0 \\
0 \\
0 \\
0\end{array}$ & $\stackrel{2}{\tilde{0}}$ & ले & $\begin{array}{l}2 \\
0 \\
0\end{array}$ \\
\hline 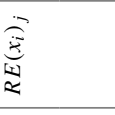 & - & & 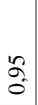 & & & - & & - & $\begin{array}{l}\Delta \\
\text { gे }\end{array}$ & - & - & - & $\stackrel{\infty}{\circ}$ & - & & $\stackrel{n}{\sigma}$ & & & - & \\
\hline \multirow[t]{2}{*}{ 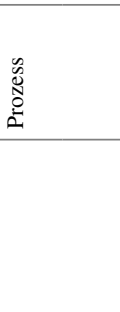 } & 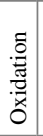 & 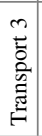 & 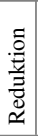 & 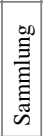 & 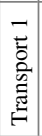 & 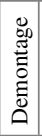 & 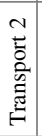 & 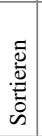 & 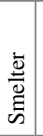 & 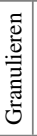 & 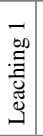 & 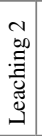 & 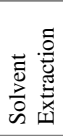 & 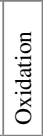 & 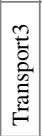 & 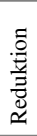 & 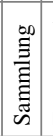 & 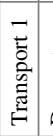 & 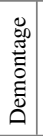 & 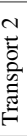 \\
\hline & & & & & 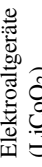 & & & & & & & & & & & & $\frac{9}{2}$ & 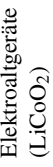 & & \\
\hline
\end{tabular}




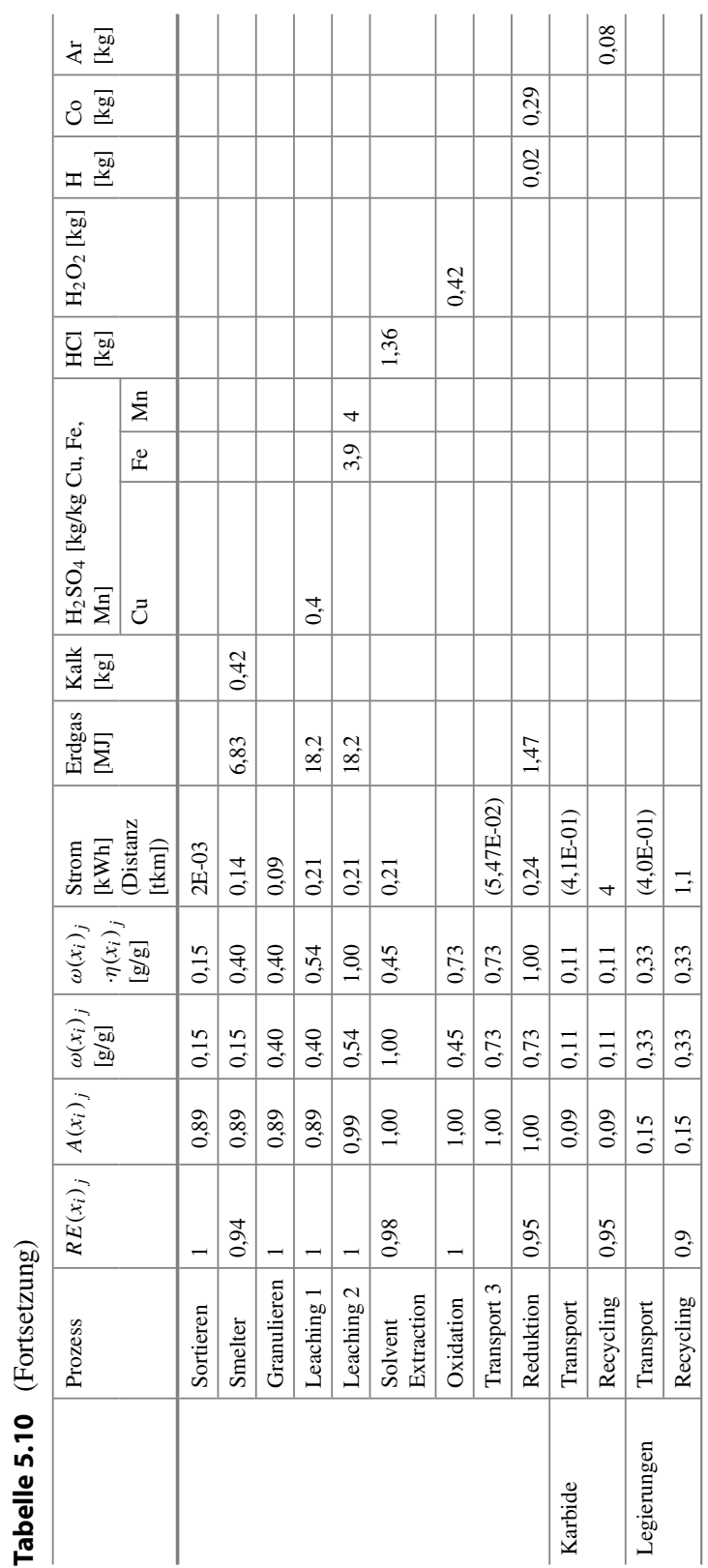


Hoboken transportiert (Transport 2/2). Die Aufbereitung in Hoboken endet mit der Oxidation zu Kobaltoxid. Die Anlage der Umicore NV/SA zur Reduktion des Oxids zu hochreinem metallischem Kobalt steht im nur wenige Kilometer entfernten Olen (Belgien). Da dieser letzte Aufbereitungsschritt, wie oben erläutert, in der Praxis nicht stattfindet, ist dieser Transport (Transport 3) fiktiv. Mit einer Distanz von ca. 5,5 km und einer sehr hohen Kobaltkonzentration von über $70 \%$ fällt dieser Transport jedoch auch nur sehr gering ins Gewicht (siehe Tabelle 5.10).

Die Transportdistanzen der Karbide und Legierungen entsprechen denen des Tantalrecyclings (Abschnitt 5.3.3.1). Für alle Transporte - mit Ausnahme der Sammlungen von Elektroaltgeräten und Altautos, die mit dem PKW durch den Endkonsumenten vorgenommen werden - sind LKW angenommen.

\subsubsection{Modellergebnisse}

Das Umberto-Modell des Kobaltrecyclings aus den untersuchten sekundären Quellen ist in Abbildung 5.20 dargestellt. Die Breite der Sankey-Pfeile gibt die Höhe der KEA an. Die Aufwände der sekundärquellenspezifischen Recyclingverfahren sind auf jeweils $1 \mathrm{~kg}$ Sekundärkobalt skaliert. Eine Beschreibung des Aufbaus eines solchen Umberto-Modells kann Abschnitt 5.2.3.2 entnommen werden.

Das Recyclingmodell in Abbildung 5.20 dient in erster Line der Veranschaulichung der Komplexität des Kobaltrecyclings aus Batterien. Von der sekundären Quelle bis zum raffinierten Sekundärkobalt werden 13, bei Altautos 14 Prozessschritte benötigt. Nur einer bzw. zwei der Prozessschritte - die Demontageprozesse - erhöhen die Kobaltkonzentration über ein mechanisches Verfahren. Alle weiteren Prozesse, die gezielt die Kobaltkonzentration erhöhen, sind metallurgische Verfahren. Die Demontageprozesse benötigen dabei verhältnismäßig geringe KEA. Das ist im Wesentlichen darauf zurückzuführen, dass die Batterien durch ihren relativ großen Anteil am gesamten EoL-Produkt meist verhältnismäßig leicht zu demontieren sind. Zudem werden bei den Demontageprozessen zahlreiche weitere Metalle demontiert, die entsprechend geringe Allokationsfaktoren für das Kobaltrecycling bedingen. Die metallurgischen Prozessschritte benötigen hingegen relativ hohe KEA. Die größten Anteile dieser KEA sind bei der Pyrowie auch der Hydrometallurgie auf den Erdgasbedarf zurückzuführen, der für die Wärmebereitstellung benötigt wird. Auch über die metallurgischen Prozessschritte werden mehrere Metalle bzw. Metallfraktionen ausgebracht, sodass die hohen KEA Inputs $\left(K E A_{D}\left(x_{i}\right)_{j}\right)$ über entsprechende Allokationen auf die Outputs verteilt werden. Dass wesentliche Anteile der $K E A_{D}\left(x_{i}\right)_{j}$ den Kuppelprodukten zugerechnet werden, ist in Abbildung 5.20 insbesondere im vorletzten Prozessschritt der Hydrometallurgie - der Solvent Extraction - zu erkennen. Hier wird 


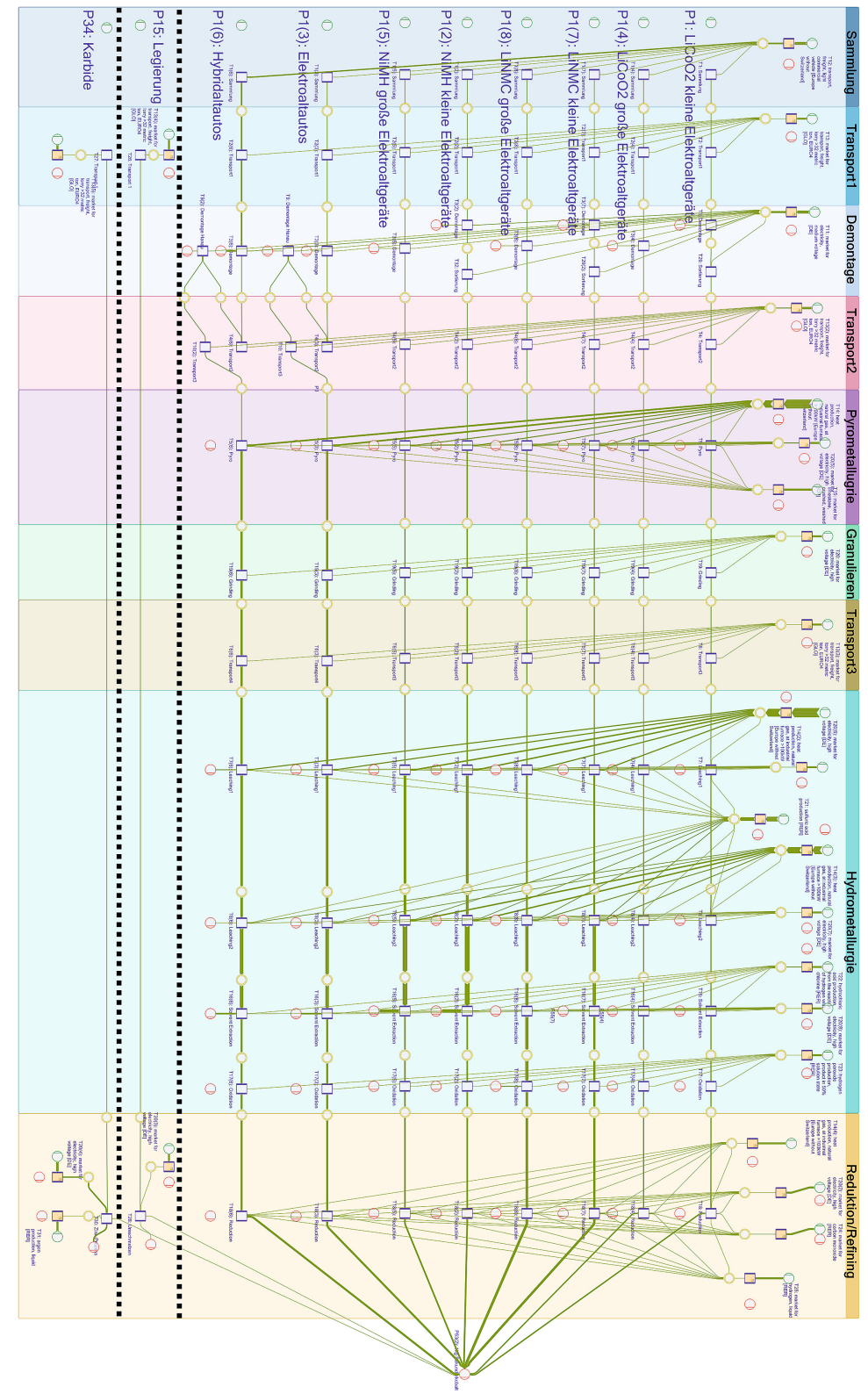

Abbildung 5.20 Umberto-Modell des Kobaltrecyclingsystems (Sankey-Darstellung der KEA, alloziert) 
Nickel, was meist mindestens doppelt so hoch in den Batterien konzentriert ist wie Kobalt, chemisch ausgelöst. Ein entsprechend hoher Anteil der $K E A_{D}\left(x_{i}\right)_{j}$ wird hier dem Recycling von Nickel zugerechnet, was durch die Pfeile in die entsprechenden Outputstellen gekennzeichnet ist.

Die Sankey-Darstellung in Abbildung 5.20 zeigt deutlich, dass das Kobaltrecycling aus Batterien die größten KEA pro kg Sekundärkobalt $K E A_{S}\left(x_{i}\right)$ aufweisen. Das Recycling von Legierungen und Karbiden benötigt verhältnismäßig geringe $K E A_{S}\left(x_{i}\right)$ von 7 und $40 \mathrm{MJ} / \mathrm{kg}$ Co. Die größten Aufwände fallen auf das Recycling der LiNMC Batterien mit $501 \mathrm{MJ} / \mathrm{kg}$ Co für kleine und $479 \mathrm{MJ} / \mathrm{kg}$ Co für große Elektroaltgeräte. Die sekundären Quellen Elektroaltautos und Hybridaltautos benötigen ebenfalls einen $K E A_{S}\left(x_{i}\right)$ von über $400 \mathrm{MJ} / \mathrm{kg}$ Co. ${ }^{14}$ Der geringste $K E A_{S}\left(x_{i}\right)$ des Kobaltrecyclings aus Batterien entfällt auf die $\mathrm{LiCoO}_{2}$-Batterien in großen Elektroaltgeräten. Eine Darstellung der prozessspezifischen $K E A_{S}\left(x_{i}\right)$ des Kobaltrecyclings aus den untersuchten sekundären Quellen ist in Abbildung 5.21 gegeben. Eine tabellarische Auflistung der Modellergebnisse, ausgewertet nach KEA und $\mathrm{CO}_{2}$ eq-Emissionen, jeweils mit und ohne Allokationen sind in Kapitel C.3 des Appendix III des elektronischen Zusatzmaterials aufgeführt.

Die Sammlung, der Transport 1 und die Demontage haben verhältnismäßig geringe Einflüsse auf den gesamten KEA des Kobaltrecyclings aus Batterien. Da sich der zentrale Modellparameter (Konzentration der Batterie an den EoLProdukten) der kleinen und großen Elektroaltgeräte nur in diesen Prozessen unterscheidet, liegen auch deren $K E A_{S}\left(x_{i}\right)$ bei gleicher Batterietechnologie (LiNMC, NiMH etc.) sehr nahe beieinander. Eine produktscharfe Betrachtung, die nach der Argumentation in Abschnitt 5.4.3.1 aufgrund einer unzureichenden Datenlage nicht durchgeführt werden kann, würde somit zu keinen wesentlich unterschiedlichen Ergebnissen führen.

Die Ergebnisse in Abbildung 5.21 zeigen, dass das Recycling von Kobalt aus Batterien kleiner Elektroaltgeräte bei gleicher Batterietechnologie höhere Demontageaufwände aufweist als das Recycling aus großen Geräten. Da die weiteren Aufbereitungsprozesse für beide Größen der Elektroaltgeräte dieselben Parameterausprägungen aufweisen, benötigt das Recycling von Kobalt aus Batterien kleiner Elektroaltgeräte höhere $K E A_{S}\left(x_{i}\right)$, als das der großen Elektroaltgeräte. Die höheren Demontageaufwände der kleinen Elektroaltgeräte sind

\footnotetext{
${ }^{14}$ Diese Werte entsprechen der Größenordnung, die die ecoinvent-Datenbank für das Recycling von Kobalt aus Batterien angibt. Ecoinvent verweist in der Datendokumentation dabei ebenfalls auf das Umicore-Verfahren.
} 


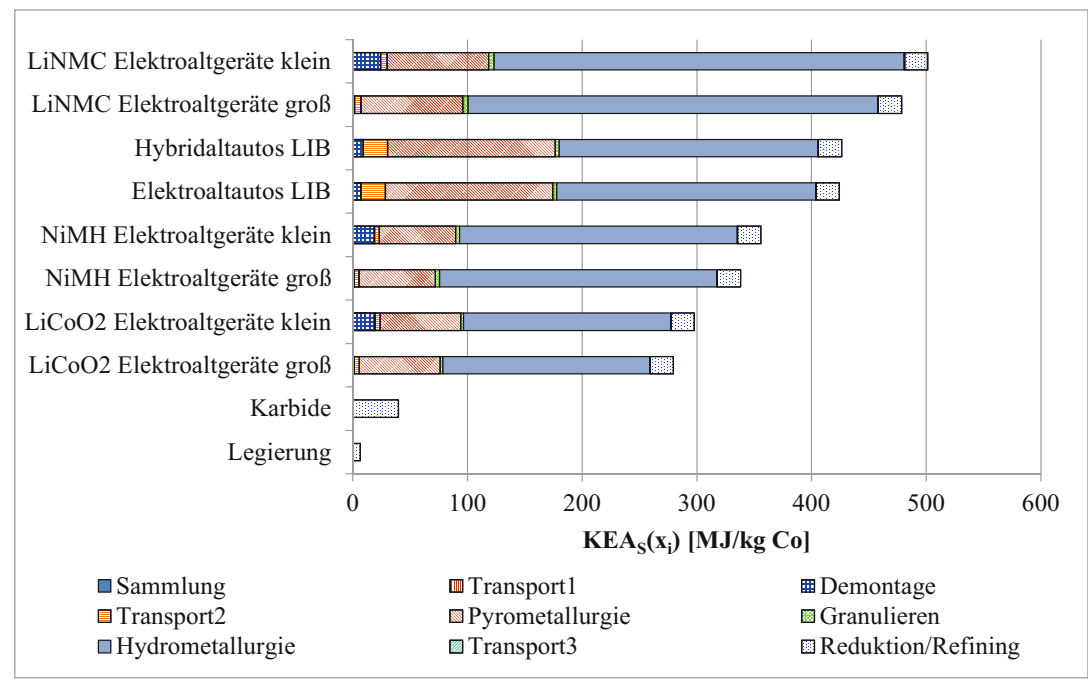

Abbildung 5.21 Allozierte prozessscharfe KEA des Kobaltrecyclings aus sekundären Quellen

dabei nicht auf Unterschiede in den Kobaltkonzentrationen zurückzuführen, sondern auf die Demontageaufwände pro Masseneinheit demontierte Elektroaltgeräte, die bei kleinen Geräten höher ausfallen als bei großen Geräten.

Der Anteil der Demontageaufwände an den $K E A_{S}\left(x_{i}\right)$ des Kobaltrecyclings ist über alle sekundären Quellen hinweg relativ gering. Dennoch findet in diesem Prozessschritt eine signifikante Erhöhung der Kobaltkonzentrationen um den Faktor 3-5 statt, bei Hybridaltautos sogar um den Faktor 31. Diese verhältnismäßig energieeffiziente Konzentrationserhöhung durch die Demontage und die Allokationseffekte der metallurgischen Prozesse schwächen den Einfluss der Kobaltkonzentrationen auf den $K E A_{S}\left(x_{i}\right)$ des Recyclings entscheidend ab. Der Zusammenhang zwischen KEA und Kobaltkonzentration weist zwar mit einem Bestimmtheitsmaß von $\mathrm{R}^{2}=0,57$ noch eine mittlere bis hohe Korrelation auf, liegt damit jedoch deutlich unter denen des Tantal- und Kupferrecyclings. Die Korrelationsanalyse ist in Abbildung 5.22 enthalten.

Über die zukünftigen Entwicklungen der Materialzusammensetzungen der kobalthaltigen Batterien gibt es bis dato zwar einige wissenschaftliche Studien, jedoch lässt sich kein eindeutiger Trend in der Entwicklung der Kobaltkonzentrationen erkennen (siehe u. a. Fergus 2010; Scrosati und Garche 2010). In 


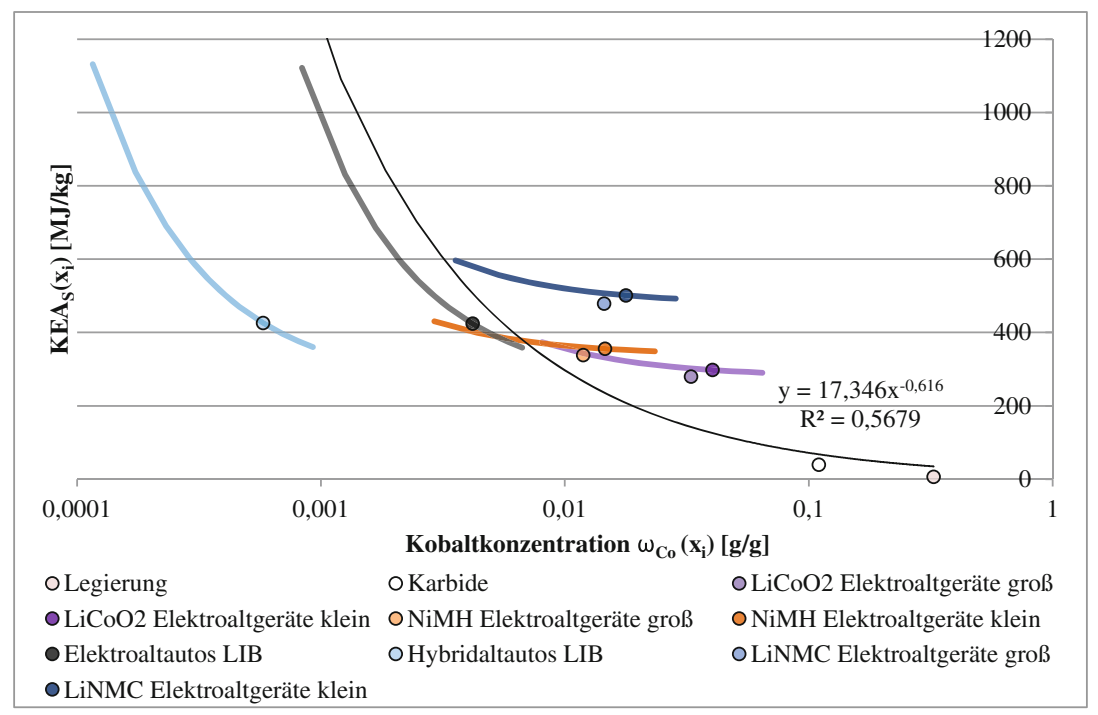

Abbildung 5.22 Auswertung der KEA des Kobaltrecyclings nach den Konzentrationen (KEA der Elektroaltgeräte nach Konzentration der Batterien und KEA der Altautos nach Kobaltkonzentration in LIB parametrisiert)

populäreren Medien finden sich jedoch fundierte Aussagen darüber, dass insbesondere bei Batterien für die automobile Anwendung Bemühungen getätigt werden, um den Kobaltgehalt zu senken. Dem Elektroautohersteller Tesla sei dies bereits in nennenswertem Umfang gelungen (Süddeutsche Zeitung 2010; WiWo 2018). Wie sich der $K E A_{S}\left(x_{i}\right)$ des Kobaltrecyclings aus LIB von Elektround Hybridautos bei Änderungen der Kobaltkonzentration der Batterie verhält, ist in Abbildung 5.22 analysiert. Diese Abbildung gibt auf der Abszisse zwar die Kobaltkonzentration der sekundären Quelle an, variiert ist jedoch nur die Kobaltkonzentration in der LIB. Der Anteil der LIB am Altauto ist als konstant angenommen. ${ }^{15}$

In den vergangenen Jahren war zudem zu beobachten, dass insbesondere LIB Batterien vermehrt Einsatz in kleinen Anwendungen finden (Al Barazi et al. 2018). Die Forschung und Entwicklung arbeitet parallel weiter mit Hochdruck

\footnotetext{
${ }^{15}$ Es gelten die gleichen Annahmen wie in Abschnitt 5.2.3.2 definiert. Konstante Allokationsfaktoren und Recyclingeffizienzen.
} 
an der Miniaturisierung von LIB, um das Einsatzgebiet auf noch kleinere Anwendungen zu erweitern (Lindinger 2018). Um die Auswirkungen dieser Entwicklung auf den KEA des Recyclings abzuschätzen, sind für kleine Elektroaltgeräte die Anteile der Batterien variiert. Diese Auswertung ist ebenfalls in Abbildung 5.22 integriert.

Die Anteile der Batterien an kleinen Elektroaltgeräten sind so variiert, dass die Gesamtkobaltkonzentrationen der sekundären Quellen eine Bandbreite von -80 \% bis $+60 \%$ abdecken. Diese Änderungen der Anteile der Batterien an den Elektroaltgeräten beeinflussen ausschließlich die Prozesse der Sammlung, des Transports 1 und der Demontage. Die vorgenommenen Änderungen haben daher nur geringe Einflüsse auf die $K E A_{S}\left(x_{i}\right)$ des Kobaltrecyclings. Diese variieren um lediglich $+25 \%$ bis $-3 \%$. Auch die Kobaltkonzentrationen in den LIB der Altautos sind um $-80 \%$ bis $+60 \%$ variiert. Die Auswirkungen dieser Konzentrationsänderungen auf die $K E A_{S}\left(x_{i}\right)$ sind mit $+165 \%$ bis $-16 \%$ signifikant. Das ist darauf zurückzuführen, dass die Kobaltkonzentrationen der LIB nicht nur die Sammlung, den Transport 1 und die Demontage beeinflussen, sondern zudem auch den Transport 2 und die Pyrometallurgie. Änderungen der Kobaltkonzentrationen innerhalb der Batterien beeinflussen die $K E A_{S}\left(x_{i}\right)$ des Kobaltrecyclings also wesentlich stärker als Änderungen der Anteile der Batterien an den EoL-Produkten.

\subsubsection{Massenbilanzen}

Die Massenbilanzen des EoL-Kobaltrecyclings basieren auf dem Jahr 2017 dem aktuellsten Jahr, für das eine ausführliche Datendokumentation existiert. Die jährlich in Deutschland gesammelten Mengen an Batterien aus Elektroaltgeräten werden regelmäßig vom Bundesministerium für Umwelt, Naturschutz und nukleare Sicherheit (BMU) ausgewiesen (BMU 2017), allerdings nur auf einer stark aggregierten Ebene. Differenziertere Daten sind für einige Sammelstellen verfügbar (z. B. GRS (2018)). Diese differenzierten Daten ermöglichen die Ermittlung der prozentualen Zusammensetzungen der Sammelmengen, die wiederum auf die deutschlandweit gesammelten Massen des BMU übertragen werden können (siehe auch Abschnitt B.3.1.1.1 in Appendix II des elektronischen Zusatzmaterials). Die anfallenden Massen an EoL-Batterien sind von der Urban Mining Plattform (2018) ausgewiesen.

Des Weiteren kann der Urban Mining Plattform entnommen werden, welche Massen an EoL-LIB aus Altautos anfallen. Welche Anteile dieser anfallenden Massen tatsächlich für ein Recycling gesammelt werden, muss abgeschätzt werden, da hierzu bis dato keine Daten zur Verfügung stehen. Es ist bekannt, dass 
Autos mit Verbrennungsmotoren nach ihrer Stilllegung zu hohen Anteilen exportiert werden - insbesondere in das nicht-europäische Ausland (siehe Abschnitt 5.2.4). Da jedoch bei Elektroautos eine entsprechende Infrastruktur für deren Nutzung benötigt wird und diese in den typischen Exportländern (insb. Afrika) nicht gegeben ist, kann davon ausgegangen werden, dass bei Elektro- bzw. Hybridaltautos solche Exporte nicht bzw. nur in sehr geringem Umfang stattfinden. Die Sammelquote der EoL-Autobatterien ist daher auf $90 \%$ geschätzt. Ein sogenannter Second Use, der eine weitere Nutzung der Batterie als stationären Stromspeicher vorsieht, ist häufig Inhalt der politischen und wissenschaftlichen Diskussionen um Batterien aus Elektroautos (siehe u. a. Reinhardt et al. 2019; Lih et al. 2012; Martinez-Laserna et al. 2018). Nach einer Experteneinschätzung kann dieser Aspekt, der im Grunde nur in einer Zeitverzögerung der anfallenden Massen resultieren würde, aktuell noch vernachlässigt werden (Weyhe 2018b).

Die Daten der gesammelten Elektroaltgeräte-Batterien beziehen die Recyclingeffizienzen der Demontage der Batterien bereits mit ein (BMU 2017; GRS 2018). Eine Batterie gilt damit nach den Angaben der referenzierten Organisationen erst als gesammelt, wenn sie aus dem EoL-Produkt entnommen bzw. demontiert ist. Welche Anteile der Differenz zwischen den angefallenen und den gesammelten Massen auf unzureichende Sammlung der sekundären Quellen und welche auf eine unzureichende bzw. nicht vorgenommene Demontage zurückzuführen sind, kann nur annähernd abgeschätzt werden. Dafür ist die Sammelquote für Elektroaltgeräte aus dem IKT-Bereich von $54 \%$ für alle Elektroaltgeräte angesetzt (Chancerel 2010).

Die ermittelten Massenbilanzen des Kobaltrecyclings sind in Abbildung 5.23 in einem Sankey-Diagramm veranschaulicht. Die Breite der Pfeile gibt die Höhe der Kobaltmassen an. Die Pfeile der sekundären Quellen Karbide und Legierungen sind aus Gründen der Sichtbarkeit hochskaliert. Die größten Verluste fallen nach der oben beschriebenen Abschätzung bei der Sammlung der Elektroaltgeräte an. Die Verluste durch eine fehlende Demontage der Batterie aus den gesammelten Elektroaltgeräten bewegen sich in der gleichen Größenordnung. ${ }^{16}$ In Summe gehen in diesen beiden Prozessschritten 73-84 \% der theoretisch verfügbaren Kobaltmassen aus Elektroaltgeräten verloren. Dennoch ist Deutschland damit im weltweiten Vergleich einer der Vorreiter. So finden in Nordamerika nur ca. $5 \%$ (Heelan et al. 2016) und in China sogar weniger als $5 \%$ der Altbatterien den Weg in ein angemessenes Recyclingverfahren ( $\mathrm{Gu}$ et al. 2017). Als wesentlicher

\footnotetext{
${ }^{16}$ Fehlende Demontagen sind in der Modellbildung nicht als Recyclingeffizienzen verstanden, sondern als nicht stattfindende Demontage. D. h. EoL-Produkte, die keiner Demontage unterzogen werden, sind auch nicht betrachtet.
} 


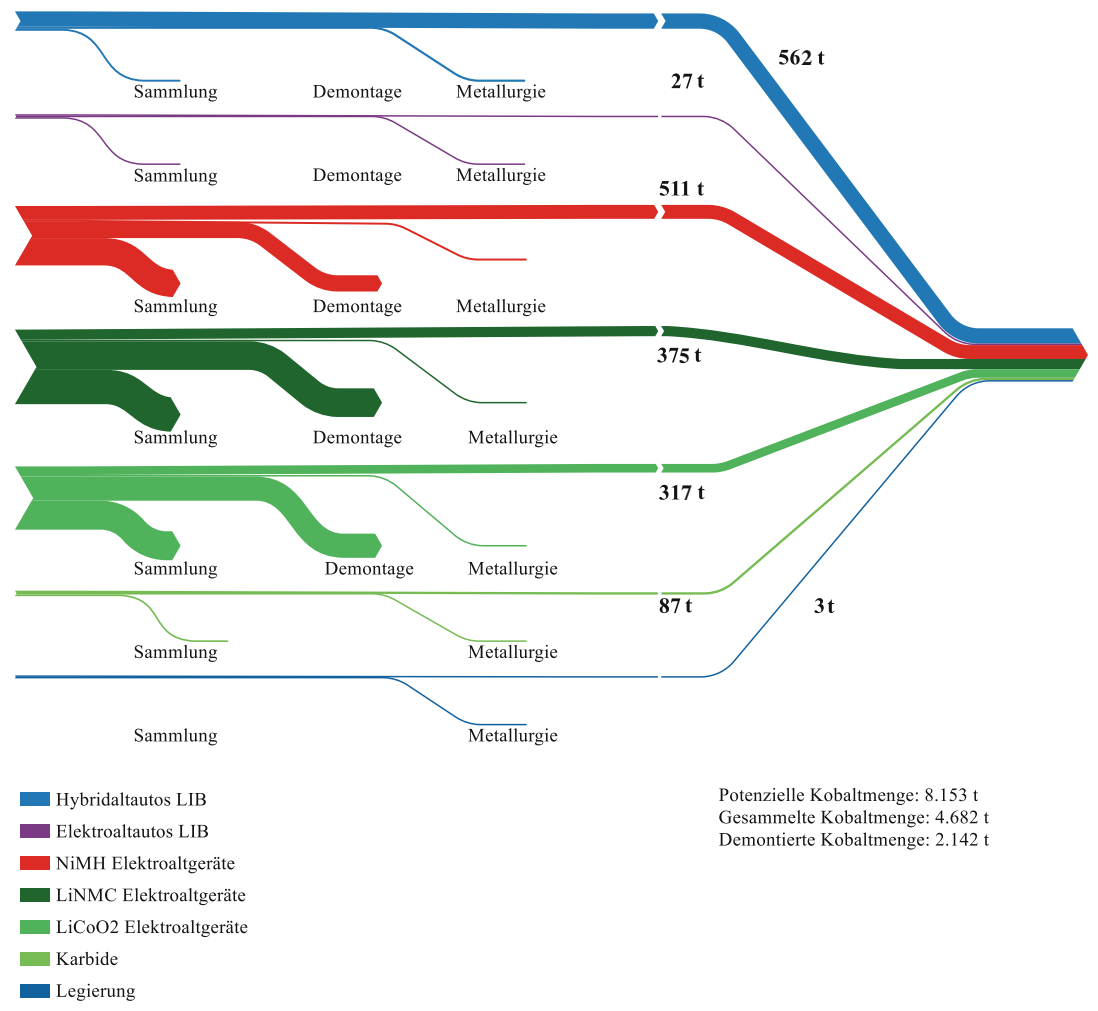

Abbildung 5.23 Massenbilanz des Kobaltrecyclings aus sekundären Quellen für das Jahr 2017 in Deutschland

Grund für die verhältnismäßig hohe innerdeutsche Sammel- und Demontagequote kann die europäische Richtlinie zur Sammlung und Verwertung von Altbatterien, die seit 2016 eine Sammelquote von mindestens $45 \%$ vorschreibt (Europäisches Parlament 2013), verstanden werden.

Durch die metallurgischen Prozessschritte gehen in Summe nochmals 12,5\% des Kobalts in Batterien verloren. Dennoch sind die einzelnen Prozessschritte der Metallurgie mit mindestens $95 \%$ Recyclingeffizienz $R E\left(x_{i}\right)_{j}$ kaum mit Materialverlusten verbunden (siehe Tabelle 5.10) und bieten daher wenig Spielraum für Verbesserungen. 
Der Großteil des theoretisch verfügbaren bzw. in sekundären Quellen anfallenden Kobalts und des Sekundärkobalts ist derzeit auf Batterien aus Hybridaltautos und Elektroaltgeräten zurückzuführen. Karbide, Legierungen und Elektroaltautos machen aktuell nur wenige Prozent der theoretisch verfügbaren Kobaltmenge und des gewonnenen Sekundärkobalts aus. In Zukunft wird der Einsatz von Kobalt in Batterien - insbesondere der Einsatz in Elektroautos - einen deutlichen Zuwachs um den Faktor 92 erfahren (Marscheider-Weidemann et al. 2016). Der massenbilanzielle Schwerpunkt der sekundären Kobaltquellen wird also zukünftig noch stärker als bisher auf den Batterien liegen.

\subsubsection{Vergleich der primären und sekundären Gewinnung}

\subsubsection{Aufwände der Primärgewinnung}

In Abschnitt 5.4.1 ist bereits darauf verwiesen, dass Kobalt zu $16 \%$ im Kleinbergbau und zu den verbleibenden $84 \%$ im industriellen Bergbau gewonnen wird. Der Schwerpunkt liegt dabei auf der DR Kongo. Die primäre Gewinnung von Kobalt erfolgt fast ausschließlich als Kuppelprodukt. Schätzungen zufolge stammen $61 \%$ aus dem Kupferbergbau und $37 \%$ aus dem Nickelbergbau. Lediglich zu $2 \%$ wird Kobalt derzeit als Hauptprodukt gefördert (Al Barazi et al. 2018).

Zum Kleinbergbau existieren aufgrund des meist händischen Abbaus keine Daten zu den Energiebedarfen. Für die industrielle Kobaltgewinnung stehen drei Datensätze, die die KEA und die THGE über den gesamten Gewinnungsprozess hinweg abdecken, zu Verfügung. Die Datenbanken ecoinvent und GaBi beinhalten jeweils einen Datensatz zur primären Kobaltgewinnung. Ein weiterer Datensatz ist vom Cobalt Development Institute (CDI) in Zusammenarbeit mit der thinkstep AG (GaBi-Datenbank Betreiber) erarbeitet. Die in den jeweiligen Datensätzen enthaltenen KEA und $\mathrm{CO}_{2}$ eq-Emissionen sind in Tabelle 5.11 aufgelistet. Je nach Datensatz fallen die Werte fundamental unterschiedlich aus. Der KEA des Datensatzes des CDI ist mit $1.430 \mathrm{MJ} / \mathrm{kg}$ Co um den Faktor 10 höher als der Wert des ecoinvent-Datensatzes. Auch die $\mathrm{CO}_{2}$ eq-Emissionen weichen um den Faktor 3,8 ab. Dass die Werte der KEA eine wesentlich größere Spannweite aufweisen als die der $\mathrm{CO}_{2}$ eq-Emissionen, ist auf den Anteil der Erneuerbaren Energien am KEA zurückzuführen. So nimmt der CDI-Datensatz einen Anteil an Erneuerbaren Energien von knapp $50 \%$ an (thinkstep AG 2012a).

Der ecoinvent-Datensatz basiert auf zahlreichen Annahmen und nur wenigen Primärdaten aus der Praxis. Zudem ist keine Auskunft darüber gegeben, welcher Anteil der globalen Kobaltproduktion von diesem Datensatz erfasst ist (ecoinvent 2013; Hischier 2007, 46ff). Der GaBi-Datensatz verweist auf eine Abdeckung 
Tabelle 5.11 KEA und $\mathrm{CO}_{2}$ eq-Emissionen der primären Kobaltgewinnung

\begin{tabular}{l|l|l}
\hline & KEA $[\mathrm{MJ} / \mathrm{kg} \mathrm{Co}]$ & $\mathrm{CO}_{2}$ eq-Emissionen $[\mathrm{kg} / \mathrm{kg} \mathrm{Co}]$ \\
\hline ecoinvent (2013) & 133 & 10 \\
\hline thinkstep AG (2017a) (GaBi) & 388 & 24 \\
\hline $\begin{array}{l}\text { thinkstep AG (2012a) (GaBi); CDI } \\
\text { (2016) }\end{array}$ & 1.430 & 38 \\
\hline
\end{tabular}

der globalen Produktion von $78 \%$, über die Datenherkunft und Qualität kann jedoch keine Aussage getroffen werden. In der begleitenden Dokumentation ist lediglich darauf verwiesen, dass der Datensatz in Kooperation mit dem CDI über eine bessere Datenqualität verfügt (thinkstep AG 2017b). Dieser Datensatz des CDI beinhaltet für das Jahr 2012 Primärdaten von acht Mitgliedern des CDI, welche die komplette Produktionsroute vom Bergbau bis zur Raffination abdecken. Dabei sind fünf Bergbauländer (inkl. DR Kongo) und sieben Länder, in denen die metallurgische Aufbereitung stattfindet, abgedeckt (inkl. Belgien (Umicore NV/SA)). Die Daten wurden entsprechend der Massenanteile der Unternehmen an der Gesamtkobaltproduktion gewichtet. Der Datensatz deckt damit $30 \%$ der Weltproduktion des Jahres 2012 ab (CDI 2016; thinkstep AG 2012b).

Basierend auf den verfügbaren Informationen ist anzunehmen, dass der CDIDatensatz die valideste Datenbasis bietet. Allerdings deckt er einen nur geringen Anteil der globalen Produktion ab. Diesen Datensatz als globalen Durchschnittswert anzusetzen, ist somit mit entsprechenden Unsicherheiten behaftet. Zudem ist es, basierend auf den verfügbaren Datensatz-Dokumentationen, nicht möglich, die beträchtlichen Abweichungen der KEA und $\mathrm{CO}_{2}$ eq-Emissionen der drei Datensätze zu erklären. Die Auswahl einer dieser Datensätze wäre also mit hohen Unsicherheiten verbunden. Für die nachfolgenden Auswertungen sind daher alle drei Datensätze verwendet.

Aktuellen Prognosen zufolge wird die globale Kobaltnachfrage zwischen 2018 und 2026 jährlich um $6 \%$ bis 8,2 \% steigen (Al Barazi et al. 2018). Auf der Angebotsseite sind entsprechende Kapazitätserweiterungen zu beobachten. Al Barazi et al. (2018) verweisen auf die Datenbank der S\&P Global Market Intelligence, die weltweit 1.091 Bergbauprojekte aufführen, die mit Kobalt assoziiert sind. 120 davon sind bereits in der Umsetzung oder in einer ernstzunehmenden Planungsphase. Der umfangreiche Überblick von Al Barazi et al. (2018) über die größten dieser Bergbauprojekte lässt jedoch keine wesentlichen Änderungen in den Gewinnungsmethoden oder Erzzusammensetzungen erkennen. Auch zukünftig wird der Großteil des primär gewonnenen Kobalts als Kuppelprodukt des 
Nickel- und Kupferbergbaus gefördert (Al Barazi et al. 2018). Es kann angenommen werden, dass der KEA der primären Kobaltgewinnung - abgesehen von grundsätzlichen technischen Effizienzsteigerungen - kurz- bis mittelfristig keinen wesentlichen Änderungen unterliegt.

\subsubsection{Ermittlung der energetisch optimalen End-of-Life Recyclingquote}

\subsection{Status Quo}

Das Recycling von Kobalt wird aktuell aus allen untersuchten sekundären Quellen praktiziert. Das gewichtete arithmetische Mittel des KEA des Kobaltrecyclings $\left(\overline{K E A}_{S}\right)$ beträgt für den Untersuchungsrahmen Deutschland und das Jahr 2017 $373 \mathrm{MJ} / \mathrm{kg} \mathrm{Co}$, bei einer EoL-RQ von $23 \%$. Auf $1 \mathrm{~kg}$ Sekundärkobalt entfallen dadurch $18 \mathrm{~kg} \mathrm{CO}_{2}$ eq-Emissionen. In Tabelle 5.12 ist aufgelistet, wie sich $\operatorname{der} \overline{K E A}_{S}$ aus den jeweiligen $K E A_{S}\left(x_{i}\right)$ der untersuchten sekundären Quellen zusammensetzt.

Tabelle 5.12 Durchschnittlicher KEA des EoL-Kobaltrecyclings

\begin{tabular}{l|l|l|l}
\hline Sekundäre Quelle $x_{i}$ & $\begin{array}{l}\text { Sekundärkobalt } \\
{[\mathrm{t}]}\end{array}$ & $K E A_{S}\left(x_{i}\right)[\mathrm{MJ} / \mathrm{kg} \mathrm{Co}]$ & $\begin{array}{l}(\text { Absoluter Anteil } \\
\frac{\mathrm{an})}{K E A_{s}}[\mathrm{MJ} / \mathrm{kg} \mathrm{Co}]\end{array}$ \\
\hline $\begin{array}{l}\text { LiNMC Elektroaltgeräte } \\
\text { klein }\end{array}$ & 167 & 501 & $(44)$ \\
\hline $\begin{array}{l}\text { LiNMC Elektroaltgeräte } \\
\text { groß }\end{array}$ & 208 & 478 & $(53)$ \\
\hline Hybridaltautos LIB & 562 & 426 & $(127)$ \\
\hline Elektroaltautos LIB & 27 & 424 & $(6)$ \\
\hline $\begin{array}{l}\text { NiMH Elektroaltgeräte } \\
\text { klein }\end{array}$ & 44 & 356 & $(8)$ \\
\hline $\begin{array}{l}\text { NiMH Elektroaltgeräte } \\
\text { groß }\end{array}$ & 467 & 338 & $(84)$ \\
\hline $\begin{array}{l}\text { LiCoO } 2 \text { Elektroaltgeräte } \\
\text { klein }\end{array}$ & 78 & 298 & $(12)$ \\
\hline $\begin{array}{l}\text { LiCoO } \\
\text { groß Elektroaltgeräte }\end{array}$ & 239 & 280 & $(35)$ \\
\hline Karbide & 87 & 40 & $(2)$ \\
\hline Legierung & 3 & 7 & $(0)$ \\
\hline Summe & 1.882 & & 373 \\
\hline
\end{tabular}


Bis zu welcher EoL-RQ das Recycling von Kobalt aus den sekundären Quellen aus energetischer Sicht optimal ist, kann aufgrund der hohen Datenvarianz der KEA der primären Gewinnung nicht eindeutig bestimmt werden. Die unterschiedlichen Datensätze der primären Kobaltgewinnung (siehe Abschnitt 5.4.5.1) führen zu fundamentalen Unterschieden in den KEA-optimierten EoL-RQ. Die Optimierung nach dem KEA des ecoinvent-Datensatzes führt zu einer KEA-optimierten EoL-RQ von lediglich $1 \%$. In diesem Fall wäre nur das Kobaltrecycling aus Legierungen und Karbiden energetisch vorteilhaft. Das Batterierecycling wäre demnach aus allen Elektroaltgeräten und Altautos mit wesentlich höheren Aufwänden, die sich um den Faktor 2 bis 4 bewegen, verbunden. Optimiert man nach

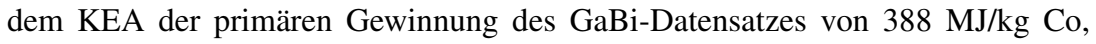
so liegt die optimale EoL-RQ bei ca. $11 \%$. Hier wäre das Kobaltrecycling aus den LIB der Altautos und den LiNMC-Batterien der Elektrogeräte mit höheren KEA als die der primären Gewinnung verbunden. Der Datensatz des CDI setzt mit $1.430 \mathrm{MJ} / \mathrm{kg}$ Co den mit Abstand höchsten KEA der primären Gewinnung an. Nach diesem Wert wäre Recycling aus allen sekundären Quellen mit energetischen Ersparnissen und einer wesentlichen Reduktion an THGE verbunden. Die KEA-optimierte EoL-RQ würde hier der aktuellen EoL-RQ von $23 \%$ entsprechen. Die grafische Optimierung nach den drei KEA der primären Kobaltgewinnung ist in Abbildung 5.24 veranschaulicht.

Nach den KEA der primären Kobaltgewinnung der Datensätze von ecoinvent und $\mathrm{GaBi}$ würde das Kobaltrecycling, wie es aktuell praktiziert wird, zu einer Erhöhung des KEA der gesamten Kobaltbereitstellung und der damit verbundenen THGE führen. Unter der Annahme des KEA des ecoinvent-Datensatzes würde die derzeitig erzielte EoL-RQ von $23 \%$ in einem Mehraufwand ${ }^{17}$ von $451 \mathrm{TJ}$ und $15.500 \mathrm{t} \mathrm{CO}_{2}$ eq-Emissionen pro Jahr resultieren. Ist der GaBiDatensatz angenommen, würden durch die EoL-RQ von $23 \%$ Ersparnisse von $29 \mathrm{TJ}$ und $11.000 \mathrm{t} \mathrm{CO}_{2}$ eq-Emissionen pro Jahr erzielt werden. Bei der nach dem GaBi-Datensatz KEA-optimierten EoL-RQ von $11 \%$ wären die Ersparnisse der KEA jedoch um $300 \%$ höher. Die Ersparnisse der THGE würden allerdings nur marginalen Änderungen unterliegen, was auf die verhältnismäßig hohen $\mathrm{CO}_{2}$ eqEmissionen der primären Gewinnung des GaBi-Datensatzes zurückzuführen ist. Dieser Datensatz gibt an, dass die Energiebereitstellung fast ausschließlich auf fossilen Energieträgern basiert (thinkstep AG 2017a). Setzt man die Aufwände des CDI an, so führt die derzeitige EoL-RQ des Kobaltrecyclings von $23 \% \mathrm{zu}$ Ersparnissen von $1.990 \mathrm{TJ}$ und $37.200 \mathrm{t} \mathrm{CO}_{2}$ eq pro Jahr.

\footnotetext{
${ }^{17}$ Mehraufwand im Vergleich zur reinen Primärgewinnung (Aufwände des Recyclings abzüglich der Aufwände der primären Gewinnung).
} 


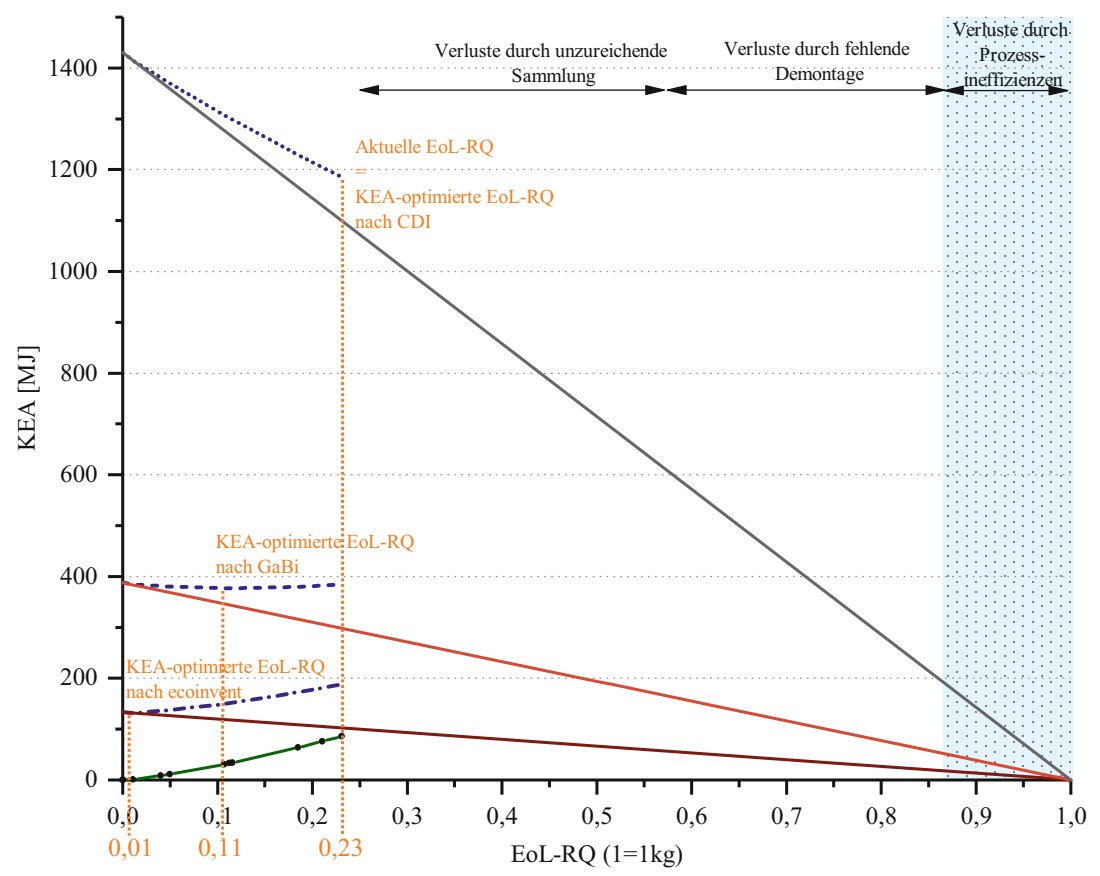

$\longrightarrow$ KEA des Recyclinganteils bei aktuellen $\mathrm{SQ}\left(\mathrm{x}_{\mathrm{i}}\right) \longrightarrow$ KEA des Primäranteils (ecoinvent) KEA des Primäranteils (GaBi) — KEA des Primäranteils (CDI) - - - • Total in MJ/kg Co (ecoinvent) -... Total in MJ/kg Co $(\mathrm{GaBi}) \cdots . .$. Total in $\mathrm{MJ} / \mathrm{kg}$ Co $(\mathrm{CDI})$

Abbildung 5.24 KEA-optimierte EoL-RQ des Kobaltrecyclings bei aktuellen Sammelquoten

\subsection{Szenarien-Analyse}

Wie im Falle des Kupfer- und Tantalrecyclings existieren auch beim EoLRecycling von Kobalt wesentliche Potenziale zur Erhöhung der EoL-RQ durch Verbesserungen der Sammelquoten $S Q\left(x_{i}\right)$. Welche Auswirkungen verbesserte $S Q\left(x_{i}\right)$ auf die KEA-optimierte EoL-RQ haben können, ist anhand zweier Szenarien analysiert, die ebenfalls für Kupfer und Tantal angewandt sind. In einem Szenario ist angenommen, dass Verbesserungen der $S Q\left(x_{i}\right)$ keinerlei Auswirkungen auf die $K E A_{S}\left(x_{i}\right)$ haben. Die Auswertung dieses Szenarios ist in Abbildung 5.25a veranschaulicht. Das zweite Szenario nimmt eine Änderung der 
Auslastung der Transportkapazitäten auf $5 \%$ und den Wechsel auf eine ökonomische Allokation für alle zusätzlich gesammelten Massen an. Dadurch ändern sich $K E A_{S}\left(x_{i}\right)$ entsprechend - siehe hierzu Abbildung 5.25b. Die grauen Datenpunkte stehen dabei für die Massen der zusätzlich gesammelten sekundären Quellen. Die Begründung des zweiten Szenarios kann Abschnitt 5.2.5.2.2 entnommen werden.

Unter der Annahme des Datensatzes der Primärgewinnung des CDI würden Änderungen in den Sammelquoten $S Q\left(x_{i}\right)$ in beiden Szenarien zu wesentlichen Steigerungen der Ersparnisse führen. Je nachdem welches Szenario angesetzt ist, belaufen sich die zusätzlichen Ersparnisse auf 1.220-1.330 TJ. Das entspricht 61-66 \% der Ersparnisse des Status Quo. Bei beiden Szenarien beträgt die optimierte EoL-RQ $56 \%$. Auch unter der Annahme des GaBi-Datensatzes können Verbesserungen der Sammelquoten $S Q\left(x_{i}\right)$ zu weiteren Reduktionen des Energiebedarfs der Kobaltgewinnung führen. Die KEA-optimierte EoL-RQ liegt nach den angenommenen Szenarien dabei im Bereich zwischen 19 und $32 \%$. Setzt man den ecoinvent-Datensatz an, so führen Verbesserungen der Sammelquoten $S Q\left(x_{i}\right)$ lediglich unter den Annahmen des ersten Szenarios (Abbildung 5.25a) zu einer marginalen Erhöhung der KEA-optimierten EoL-RQ auf $2 \%$.

Neben den geringen Sammelquoten $S Q\left(x_{i}\right)$ gehen derzeit noch große Massen an Kobalt durch nicht-stattfindende Demontage der Batterien aus Elektroaltgeräten verloren. Die Verluste fallen, den Annahmen und Auswertungen von Abschnitt 5.4.4 zufolge, ähnlich hoch aus wie die Verluste durch die unzureichende Sammlung. Der Umfang der ermittelten nicht-stattfindenden Demontage ist, aufgrund der in Abschnitt 5.4.4 getroffenen Annahme gleicher Sammelquoten für alle Elektroaltgeräte, mit Unsicherheiten verbunden. Dass die Demontierbarkeit von Batterien aus Elektroaltgeräten durch z. B. Verklebungen der Batterien stark eingeschränkt ist, bestätigen Stimmen aus der Praxis (Fröhlich 2018a). Fakt ist auch, dass die Demontage von Batterien bei weitem nicht so gut funktioniert, wie in $§ 4$ des Elektro- und Elektronikgerätegesetzes (ElektroG) angedacht. Daher werden auch strengere Vorgaben bei einer Novellierung des ElektroG gefordert (Rechenberg et al. 2019). Welcher Aufwand mit der Erhöhung der Demontagequote verbunden ist, kann aufgrund fehlender Daten nicht ermittelt werden.

Die prozesskettenübergreifenden Recyclingeffizienzen $R E\left(x_{i}\right)$ sind für alle sekundären Quellen mit ca. $90 \%$ relativ hoch und bieten daher nur geringes Potenzial für weitere Verbesserungen. Bei der Betrachtung der prozessspezifischen Recyclingeffizienzen $R E\left(x_{i}\right)_{j}$ des Kobaltrecyclings fällt auf, dass diese deutlich über $90 \%$ bzw. nur knapp unter $100 \%$ liegen (siehe Tabelle 5.10). Es kann somit davon ausgegangen werden, dass die prozessspezifischen $R E\left(x_{i}\right)_{j}$ - deren Produkt die $R E\left(x_{i}\right)$ ergibt - bereits nahe an den thermodynamisch möglichen Grenzen liegen. 

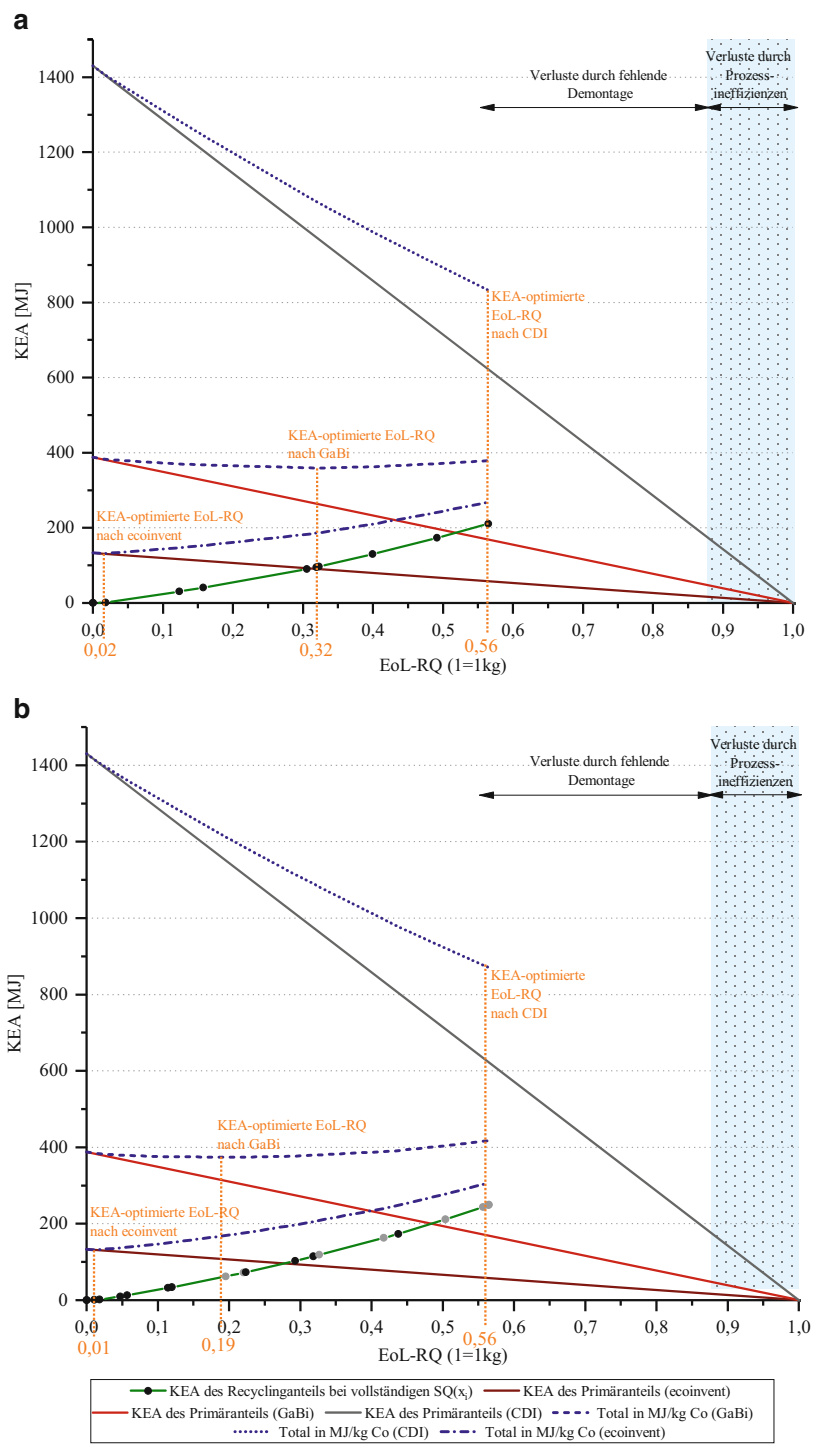

Abbildung 5.25 KEA-optimierte EoL-RQ des Kobaltrecyclings bei vollständigen Sammelquoten und a konstanten Sammelaufwänden pro kg Co bzw. b variablen Sammelaufwänden pro kg Co (5\% Auslastung der Transportkapazitäten, ökonomische Allokation) 


\subsubsection{Fazit zum Kobaltrecycling}

Die derzeitige EoL-RQ von Kobalt in Deutschland - einem der Vorreiterländer in Bezug auf Recyclingquoten - liegt nach den obigen Kalkulationen bei $23 \%$. Nach den Angaben von UNEP beträgt die globale EoL-RQ von Kobalt über $50 \%$ (UNEP 2011b). Hier ist jedoch zu beachten, dass der Zeitpunkt der Veröffentlichung dieser UNEP Studie bereits mehrere Jahre zurückliegt. Die verwendeten Daten der Studie sind also mindestens ebenfalls so alt bzw. in den meisten Fällen noch älter. Gerade bei den Anwendungsbereichen von Kobalt hat seitdem eine hohe Marktdynamik stattgefunden. So hat u. a. der Einsatz von Kobalt in Batterien in portablen Elektrogeräten einen wesentlichen Zuwachs erfahren (Al Barazi et al. 2018). Durch die geringen Sammelquoten $S Q\left(x_{i}\right)$ der daraus resultierenden sekundären Quelle der Elektroaltgeräte hat in den vergangenen Jahren also vermutlich ein Rückgang der EoL-RQ stattgefunden.

Die hohen Recyclingeffizienzen $R E\left(x_{i}\right)$ des Kobaltrecyclings aus Batterien zeigen, dass die Recyclingverfahren für die anstehenden Massen an ausgedienten LIB aus Elektro- und Hybridautos nicht nur bereits vorhanden, sondern technisch auch in hohem Maße ausgereift sind. Die einleitend erwähnte Diskussion um die EoL-Lösung der EoL-LIB ist also unbegründet. Die Kreislaufführung von Kobalt aus LIB, sowie zahlreicher weiteren darin enthaltener Metalle, wie z. B. Lithium oder Nickel, ist also technisch machbar und wird auch bereits praktiziert. Ob das Recycling jedoch energetisch vorteilhafter als die Primärgewinnung ist und damit zu Ersparnissen an THGE führt, kann derzeit nicht mit Sicherheit bestimmt werden, da die existierende Datenlage zum KEA der Primärgewinnung von Kobalt nicht valide ist. Unter der Annahme des Datensatzes des CDI, der die höchste Validität vermuten lässt, führt das Kobaltrecycling, wie es derzeit praktiziert wird, zu signifikanten Ersparnissen an Energie und THGE. Nach den beiden anderen Datensätzen, die für die primäre Kobaltgewinnung zur Verfügung stehen, würde die aktuelle EoL-RQ von $23 \%$ bereits zu negativen Trade-offs führen.

Die Inkonsistenzen in der Datenlage zur Primärgewinnung von Kobalt sind also eine zentrale Unsicherheit für die notwendige Zielausrichtung der Recyclingaktivitäten. Dieses Beispiel veranschaulicht die Notwendigkeit valider und konsistenter Daten, die die primäre und die sekundäre Gewinnung gleichermaßen betreffen. Nur anhand ausreichend valider Daten kann sichergestellt werden, dass Recyclingaktivitäten so ausgerichtet werden, dass sie zu einer Reduktion der THGE und damit zu einer nachhaltigen Entwicklung beitragen. Die enorme Spannweite der KEA der unterschiedlichen Datensätze birgt zudem hohe Unsicherheiten in der ökologischen Bewertung der Elektromobilität, die nach aktuellem Stand auch zukünftig nicht ohne Kobalt auskommen wird. 
Open Access Dieses Kapitel wird unter der Creative Commons Namensnennung 4.0 International Lizenz (http://creativecommons.org/licenses/by/4.0/deed.de) veröffentlicht, welche die Nutzung, Vervielfältigung, Bearbeitung, Verbreitung und Wiedergabe in jeglichem Medium und Format erlaubt, sofern Sie den/die ursprünglichen Autor(en) und die Quelle ordnungsgemäß nennen, einen Link zur Creative Commons Lizenz beifügen und angeben, ob Änderungen vorgenommen wurden.

Die in diesem Kapitel enthaltenen Bilder und sonstiges Drittmaterial unterliegen ebenfalls der genannten Creative Commons Lizenz, sofern sich aus der Abbildungslegende nichts anderes ergibt. Sofern das betreffende Material nicht unter der genannten Creative Commons Lizenz steht und die betreffende Handlung nicht nach gesetzlichen Vorschriften erlaubt ist, ist für die oben aufgeführten Weiterverwendungen des Materials die Einwilligung des jeweiligen Rechteinhabers einzuholen.

(c) (P) 